Filipe

Emanuel de Sá Rocha
Complementing vehicular connectivity coverage through cellular networks

Complemento da cobertura de uma rede veicular através de redes celulares

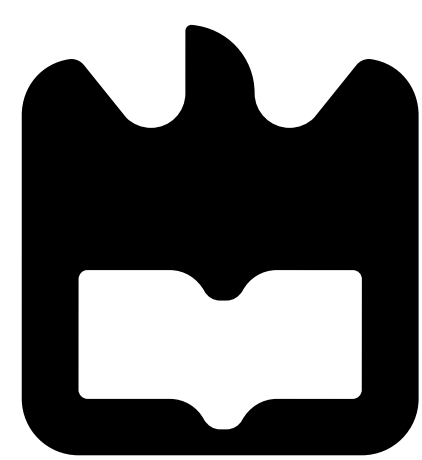



Filipe

Emanuel de Sá Rocha

\section{Complementing vehicular connectivity coverage through cellular networks}

\section{Complemento da cobertura de uma rede veicular através de redes celulares}

Dissertação apresentada à Universidade de Aveiro para cumprimento dos requisitos necessários à obtenção do grau de Mestre em Engenharia de Computadores e Telemática, realizada sob a orientação científica do Doutor Nuno Miguel Abreu Luís, Investigador Auxiliar do Instituto de Telecomunicações, e do Doutor André Ventura da Cruz Marnoto Zúquete, Professor Auxiliar do Departamento de Eletrónica, Telecomunicações e Informática da Universidade de Aveiro 

o júri / the jury

presidente / president

vogais / examiners committee

\section{Doutor Paulo Miguel Nepomuceno Pereira Monteiro}

Professor Associado do Departamento de Eletrónica, Telecomunicações e Informática da Universidade de Aveiro

\section{Doutor Pedro Miguel Salgueiro dos Santos}

Professor Auxiliar Convidado da Faculdade de Engenharia da Universidade do Porto

\section{Doutor Nuno Miguel Abreu Luís}

Investigador Auxiliar do Instituto de Telecomunicações 

agradecimentos /

acknowledgements
Quero começar por agradecer à minha família e em especial aos meus pais, Dimas e Dulce Rocha, por todo o constante apoio, carinho e incentivo que proporcionaram ao longo desta grande etapa da minha vida. Um eterno obrigado por me terem ajudado a tornar este sonho realidade.

Gostaria também de agradecer a todos os meus colegas desta vida académica pelos convívios e experiências partilhadas, esperando que o futuro traga ainda mais e melhores momentos com todos. Aproveito também para agradecer aos meus amigos de longa data Ana Rita Silva, Ricardo Oliveira, Válter Vieira, Samantha Oliveira e Luís Gameiro por me terem marcado cada um de forma tão única e especial.

Quero deixar um especial agradecimento à professora Susana Sargento e ao professor André Zúquete por esta oportunidade, pela orientação desde o primeiro dia e pelo espírito crítico tendo ambos providenciado interações fundamentais para o sucesso desta dissertação. Asim como agradeço, também, ao Doutor Miguel Luís por toda a disponibilidade (paciência) e apoio que me dedicou ao longo da realização desta dissertação, tendo sido um excelente orientador. Fico muito grato pela experiência que me permitiram adquirir ao longo deste projecto. Quero ainda agradecer a amizade e entreajuda que o grupo de investigação do laboratório de redes NAP sempre manteve, salientando o Christian Gomes e o Rui Lopes por todo o tempo e ajuda que disponibilizaram. É, sem dúvida, uma honra fazer parte deste grupo.

Gostaria também de agradecer ao Instituto de Telecomunicações e ao projeto MobiWise (POCI-01-0145-FEDER-016426) financiado através do Fundo Europeu de Desenvolvimento Regional (FEDER), através do Programa Operacional Competitividade e Internacionalização (COMPETE 2020) do Portugal 2020, e da Fundação para a Ciência e Tecnologia (FCT). 

Resumo

As redes veiculares (Vehicular Ad hoc Networks, VANETs) suportam o desenvolvimento de aplicações de segurança ou de entretenimento, permitindo que veículos comuniquem entre si e com a infraestrutura, garantindo a sua ligação e a dos seus ocupantes à Internet. Estas redes veiculares podem depender de diferentes tecnologias para a sua implementação tais como IEEE 802.11p (WAVE), IEEE 802.11a/g/n (Wi-Fi), que mesmo assim podem não garantir uma cobertura plena e total conectividade. Desta forma, esta dissertação tem como principal objectivo a criação de mecanismos de rede necessários para dotar uma rede veicular, com suporte para mobilidade e multihoming, de uma interface de comunicação adicional que faz uso de redes celulares comerciais, aumentando assim a conectividade da mesma.

O trabalho realizado ao longo desta dissertação permitiu a integração da rede celular comercial nos mecanismos de mobilidade inter-tecnologia presentes na rede veicular existente, que serviu de base para este projecto. A implementação da integração de uma solução celular permite que veículos mantenham comunicação de uma forma integrada com a Internet, mesmo quando não existe ligação disponível através das tecnologias de comunicação veiculares tradicionais, como o IEEE 802.11p. A solução proposta faz uso de redes celulares comerciais sem requerer contribuições da parte destas, e sendo completamente transparente para os utilizadores ligados à rede veicular.

A solução desenvolvida foi avaliada em cenários de laboratório e em cenários reais com sistemas reais, que permitiram avaliar o desempenho da solução proposta. Os resultados obtidos demonstram que, com a solução celular é, de facto possível manter a conectividade à rede veicular, mas em testes de elevado tráfego, o desempenho da rede celular começa a deteriorar. Foi assim possível validar o correcto funcionamento da rede veicular com a solução celular, reagindo de forma autónoma às necessidades apresentadas de forma a manter ligação entre os veículos e a infraestrutura, sem necessidade de intervenção por parte de qualquer entidade externa. 

Abstract

Vehicular Ad hoc Networks (VANETs) support the development of safety and infotainment applications, allowing vehicles to communicate between each other and with the infrastructure, assuring their connection and of its occupants to the Internet. These VANETs may rely on different technologies for their deployment such as IEEE 802.11p (WAVE) or IEEE $802.11 \mathrm{a} / \mathrm{g} / \mathrm{n}$ ( $\mathrm{Wi}-\mathrm{Fi})$, but even then complete coverage and full connectivity may not be assured. Therefore, this dissertation has the main objective of creating the necessary network mechanisms to allow the VANET, with mobility and multihoming support, to make use of an additional communication interface via commercial cellular networks, increasing its availability.

The work developed in this dissertation has allowed the integration of a commercial cellular network in the inter-technology mobility mechanisms available in the vehicular network, that served as the base work for this project. The implementation of the integration of a cellular solution allows the vehicles to maintain communication to the Internet through the VANET, even when there is no available connection through mainstream VANET technologies, such as IEEE 802.11p. The proposed solution uses commercial cellular networks without the need of any contribution by the network providers and maintaining transparency to the end-user.

The implemented approach was evaluated with real systems in laboratory and real-world scenarios, in order to evaluate the performance of the proposed solution. The obtained results show that with the cellular solution it is, in fact, possible to maintain connectivity to the VANET, but in high traffic tests the cellular network starts to underperform. Nevertheless, it was possible to validate the correct execution of the VANET with the cellular solution, automatically reacting to the respective necessities to maintain connection between the vehicles and infrastructure, without the need of intervention by any external entity in the process. 



\section{Contents}

Contents $\quad$ i

List of Figures $\quad$ iii

List of Tables $\quad v$

Acronyms $\quad$ vii

1 Introduction 1

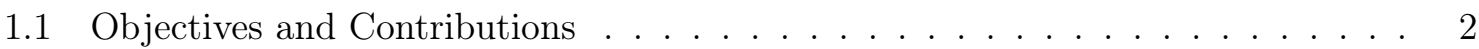

1.2 Document Organization . . . . . . . . . . . . . . . . . 2

2 State of the Art 5

2.1 Vehicular Ad-Hoc Networks . . . . . . . . . . . . . . . . . . 5

2.1 .1 Characteristics . . . . . . . . . . . . . . . . 6

2.1 .2 Architecture . . . . . . . . . . . . . . . . . 7

2.1 .3 Applications . . . . . . . . . . . . . . . . . . 7

2.1 .4 Challenges . . . . . . . . . . . . . . . . . . . . 8

2.2 Network Access Technologies . . . . . . . . . . . . . . . . . . . . . 8

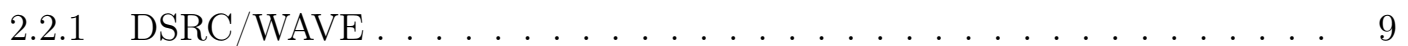

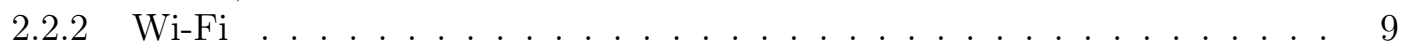

2.2 .3 Cellular . . . . . . . . . . . . . . . . . . . 9

2.3 N-PMIPv6 Mobility Protocol . . . . . . . . . . . . . . . . . . . . . 10

2.3.1 Terminology . . . . . . . . . . . . . . . . . . 11

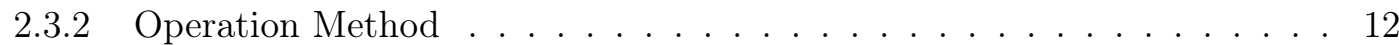

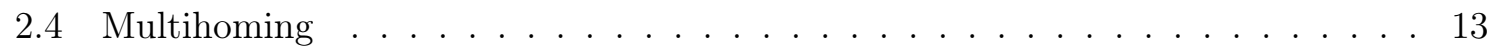

2.4.1 Proxy multihoming extension for PMIPv6 . . . . . . . . . . . . 13

2.5 Final Considerations . . . . . . . . . . . . . . . . . . . . . . 14

3 Related Work $\quad 15$

3.1 VANET Technologies and Architectures . . . . . . . . . . . . . . . 15

$3.25 \mathrm{G}$ for Vehicular Environments . . . . . . . . . . . . . . . . . 18

3.3 Final Considerations . . . . . . . . . . . . . . . . . . . . . . . . . . . . . . . . . 19

4 Mobility with Cellular Communications: Architecture 21

4.1 Current Architecture . . . . . . . . . . . . . . . . . . . . . . . 21

4.1 .1 Mobility Protocol . . . . . . . . . . . . . . . . . . . 22 
4.1.1.1 LMA Operation . . . . . . . . . . . . . . . . 22

4.1.1.2 MAG/mMAG Operation . . . . . . . . . . . . . 22

4.1.2 User Internet IPv4 connection support through OBUs . . . . . . . . . . 26

4.1.3 Connection Manager Operations . . . . . . . . . . . . . . . . . . . 27

4.1 .4 Considerations . . . . . . . . . . . . . . . . . . 27

4.2 A Multi-technology VANET Architecture . . . . . . . . . . . . . 27

4.2 .1 Overview of the Architecture . . . . . . . . . . . . . . 27

4.2 .2 Challenges . . . . . . . . . . . . . . . . . . . . . . . . . . . . . 29

4.2 .3 Proposed Approach . . . . . . . . . . . . . . . . . . . . . . . . . . . 29

4.2 .4 Overview of a Communication Scenario . . . . . . . . . . . . . . 31

4.3 Final Considerations . . . . . . . . . . . . . . . . . . . . . . . 32

5 Mobility with Cellular Communications: Implementation 33

5.1 N-PMIPv6 and Connection Manager Improvements . . . . . . . . . . . . . . . 33

5.2 Connection Manager Cellular Operation . . . . . . . . . . . . . . 34

5.2 .1 Disconnect Mechanism . . . . . . . . . . . . . . . . . . 36

5.3 LMA Cellular Operation . . . . . . . . . . . . . . . . . . . . . . 37

5.4 Heartbeat Mechanism . . . . . . . . . . . . . . . . . . . . . . . . . 39

5.5 Final Considerations . . . . . . . . . . . . . . . . . . . . . . . 39

6 Evaluation $\quad 41$

6.1 Hardware and Software . . . . . . . . . . . . . . . . . . . . . 41

6.2 Tests . . . . . . . . . . . . . . . . . . . . . . 43

6.2.1 Laboratory Tests . . . . . . . . . . . . . . . . . . . . . . 45

6.2 .2 Real-World Tests . . . . . . . . . . . . . . . . . . . . . . . . . 49

6.2.3 Analysing the performance over time . . . . . . . . . . . . . . . 51

6.2.4 Disconnect Mechanism Tests . . . . . . . . . . . . . . . . . 53

6.3 Final Considerations . . . . . . . . . . . . . . . . . 56

7 Conclusions and Future Work $\quad 59$

7.1 Future Work . . . . . . . . . . . . . . . . . . . . . . 60

$\begin{array}{ll}\text { Bibliography } & 61\end{array}$ 


\section{List of Figures}

2.1 VANET example . . . . . . . . . . . . . . . . . . . . . . 6

2.2 Types of VANET Architectures . . . . . . . . . . . . . . . . . . . 7

2.3 Cellular network representation. . . . . . . . . . . . . . . . . . 10

2.4 N-PMIPv6 protocol overview . . . . . . . . . . . . . . . . . . 11

2.5 Multihoming Architecture Example . . . . . . . . . . . . . . . . . . . 13

3.1 Communications in VANETs . . . . . . . . . . . . . . . . 16

3.2 V2X communications in a DSRC-cellular hybrid urban scenario . . . . . . . . 18

4.1 LMA operation flow diagram . . . . . . . . . . . . . . . . 23

4.2 Network Abstraction representation . . . . . . . . . . . . . . . . . . . 24

$4.3 \mathrm{MAG}$ and mMAG operation flow diagram . . . . . . . . . . . 25

4.4 User Internet IPv4 connection support . . . . . . . . . . . . . . . . . 26

4.5 Proposed VANET architecture. . . . . . . . . . . . . . . . . . 28

4.6 Example of VANET messages in a cellular communication . . . . . . . . . . . 31

4.7 Example of end-user communications via respective tunnels. . . . . . . . . . . . 31

5.1 Overview of cellular communication . . . . . . . . . . . . . 35

5.2 Connection manager cellular operation work flow . . . . . . . . . . 36

5.3 LMA cellular operation flow diagram. . . . . . . . . . . . . . . . 38

$6.1 \quad$ NetRider version 3. . . . . . . . . . . . . . . . . . . . . . . . . . . 42

6.2 Evaluation scenario $1 . \ldots \ldots \ldots \ldots \ldots \ldots$

6.3 Scenario timeline. . . . . . . . . . . . . . . . . . . . . . . 45

6.4 Packet loss in laboratory conditions . . . . . . . . . . . . . . . . . 48

6.5 Packet loss in real-world conditions . . . . . . . . . . . . . . . . . 51

6.6 Best and Worst case for test A, in scenario 1 with the Cell Solution. . . . . . . 52

6.7 Best and Worst case for test B, in scenario 1 with the Cell Solution. . . . . . . 52

6.8 Packets dropped for tests A. . . . . . . . . . . . . . . . . 56

6.9 Packets dropped for tests B. . . . . . . . . . . . . . . . . 56 


\section{List of Tables}

6.1 CN and LMA Host Equipment characteristics. . . . . . . . . . . . . . . . . . . 42

6.2 LMA (Virtual Machine) characteristics. . . . . . . . . . . . . . . . . . 42

6.3 Client equipment characteristics. . . . . . . . . . . . . . . . . . 42

6.4 NetRider Specifications. . . . . . . . . . . . . . . . . . . . . . 43

6.5 Parameters for each test. . . . . . . . . . . . . . . . . . . . . . . . . . . . . . . . . 44

6.6 Results for the Base Solution in laboratory conditions. . . . . . . . . . . . . . . 45

6.7 Results for the Cell solution in laboratory conditions. . . . . . . . . . . . . . 46

6.8 Results for 50 seconds of WAVE connection, scenario 2, in laboratory conditions. 47

6.9 Results for 15 seconds of cellular, scenario 3, in laboratory conditions. . . . . . 47

6.10 Results for the Base Solution in real-world conditions. . . . . . . . . . . . . . . 49

6.11 Results for the Cell Solution in real-world conditions. . . . . . . . . . . . . . 50

6.12 Results for 50 seconds of WAVE connection, scenario 2, in real-world conditions. 50

6.13 Results for 15 seconds of cellular, scenario 3, in real-world conditions. . . . . . 50

6.14 Results for tests with a disconnect for RSSI $<10 \ldots \ldots \ldots \ldots$. . . . . . . 54

6.15 Results for tests with a disconnect for RSSI $<20 \ldots \ldots \ldots \ldots \ldots$. . . . 54

6.16 Results for tests with a disconnect for RSSI $<30 \ldots \ldots \ldots \ldots \ldots$. . . . . 54 


\section{Acronyms}

\begin{tabular}{|c|c|}
\hline $1 G$ & First Generation \\
\hline $2 \mathrm{G}$ & Second Generation \\
\hline $3 G$ & Third Generation \\
\hline $4 \mathrm{G}$ & Fourth Generation \\
\hline $5 \mathrm{G}$ & Fifth Generation \\
\hline AP & Access Point \\
\hline AR & Access Router \\
\hline $\mathrm{BCE}$ & Binding Cache Entry \\
\hline BS & Base Station \\
\hline C-RAN & Cloud-RAN \\
\hline $\mathrm{CN}$ & Correspondent Node \\
\hline D-ITG & Distributed Internet Traffic Generator \\
\hline DDoS & Distributed Denial of Service \\
\hline DR & Delete Route \\
\hline DSRC & Dedicated Short-Range Communications \\
\hline GPS & Global Position System \\
\hline HSDPA & High Speed Downlink Packet Access \\
\hline ID & IDentifier \\
\hline IEEE & Institute of Electrical and Electronics Engineers \\
\hline IoT & Internet of Things \\
\hline IPv4 & Internet Protocol version 4 \\
\hline IPv6 & Internet Protocol version 6 \\
\hline IP & Internet Protocol \\
\hline
\end{tabular}




\begin{tabular}{|c|c|}
\hline ITS & Intelligent Transportation System \\
\hline KA & Keep Alive \\
\hline LMA & Local Mobility Anchor \\
\hline LTE & Long Term Evolution \\
\hline MAG & Mobile Access Gateway \\
\hline MANET & Mobile Ad-Hoc Network \\
\hline MIPv6 & Mobile Internet Protocol version 6 \\
\hline mMAG & mobile MAG \\
\hline MN-HNP & Mobile Node's Home Network Prefix \\
\hline MN-ID & Mobile Node IDentifier \\
\hline MN & Mobile Node \\
\hline MR & Mobile Router \\
\hline MU & Mobile User \\
\hline N-PMIPv6 & Network-Proxy Mobile Internet Protocol version 6 \\
\hline NAP & Network Architectures and Protocols \\
\hline NAT & Network Address Translation \\
\hline NEMO & NEtwork MObility \\
\hline OBU & On-Board Unit \\
\hline OS & Operating System \\
\hline PBA & Proxy Binding Acknowledgment \\
\hline PBU & Proxy Binding Update \\
\hline PDR & Packet Delivery Ratio \\
\hline PMIPv6 & Proxy Mobile Internet Protocol version 6 \\
\hline PoA & Point-of-Attachment \\
\hline Proxy-CoA & Proxy Care-of Address \\
\hline QoE & Quality of Experience \\
\hline QoS & Quality of Service \\
\hline RA & Router Advertisement \\
\hline RSSI & Radio Signal Strength Intensity \\
\hline
\end{tabular}




$\begin{array}{ll}\text { RSU } & \text { Road-Side Unit } \\ \text { RS } & \text { Router Solicitation } \\ \text { SDN } & \text { Software Defined Networking } \\ \text { SMS } & \text { Short Message System } \\ \text { UDP } & \text { User Datagram Protocol } \\ \text { V2I } & \text { Vehicle-to-Infrastructure } \\ \text { V2V } & \text { Vehicle-to-Vehicle } \\ \text { V2X } & \text { Vehicle-to-Everything } \\ \text { VANET } & \text { Vehicular Ad Hoc Network } \\ \text { VoIP } & \text { Voice over IP } \\ \text { WAVE } & \text { IEEE } 802.11 \mathrm{p} \\ \text { Wi-Fi } & \text { IEEE } 802.11 \mathrm{a} / \mathrm{b} / \mathrm{g} \\ \text { WLAN } & \text { Wireless Local Area Network }\end{array}$




\section{Chapter 1}

\section{Introduction}

Vehicular Ad Hoc Network (VANET) emerges as an exciting area of research and development in current days, in part also encouraged by the Internet of Things (IoT) revolution that is undergoing. Having the possibility to exchange messages between vehicles in a particular area, or even gathering information from sensors to improve on-road decisions, may increase vehicular traffic efficiency reducing the time spent in traffic jams. Vehicles may also have the capability to provide access to the network to its passengers, raising the comfort during the ride. Also, this will help autonomous cars, by allowing the sharing of sensor information among vehicles towards better and faster decisions. These are just a few obvious uses for this type of technology, as VANETs present a logical and needed evolution in the automotive and technological landscape.

The focus of this dissertation lies in a VANET environment with multihoming and multihop communication capabilities between vehicles and with the infrastructure for the dissemination of messages between vehicles and Internet access. This VANET makes use of the IEEE 802.11p (WAVE) and IEEE 802.11a/b/g (Wi-Fi) radio communication standards for Internet Protocol (IP) data networks.

In current days, cities have many Wi-Fi hotspots usually positioned in touristic points of interest and main social areas providing a Wi-Fi coverage. Although limited in range, they are still numerous enough to present themselves as an option to connect to the Internet. Since this will be limited to certain areas of reduced coverage, Dedicated Short-Range Communications (DSRC)/WAVE was introduced as the primary option to allow the communication between the vehicles (Vehicle-to-Vehicle (V2V) and Vehicle-to-Infrastructure (V2I)). Nevertheless, the nodes can still find themselves in areas without coverage by DSRC/WAVE or Wi-Fi. Moreover, in a way to increase the availability of the mobility platform for the users, the cellular network, a wide available communication network as seen by its global deployment and coverage areas mainly found around vehicular routes, could be of great value. With the well known and wide availability of Third Generation (3G), the continuous developments on Fourth Generation (4G) as a solid cellular technology for a vast variety of solutions such as high quality multimedia content, and with Fifth Generation (5G) technology in development and early deployment making way to a more significant evolution of cellular networks all around the world in the near future, it becomes a logical choice the implementation of cellular capabilities in VANETs. 


\subsection{Objectives and Contributions}

The VANET base approach supports multiple radio communication technologies, isolated or in parallel, but does not support communications via commercial cellular networks. Therefore, the main objective of this dissertation is to accommodate the support of those communications in the VANET, with a minimum impact on its architecture and without any modification/contribution needed by cellular network providers or end-users. As such, an overview of the goals and contributions of this dissertation are as follows:

- Study of the VANET mobility protocol and multihoming architecture: to understand the current platform in use and the possible modifications needed to integrate a new communication alternative.

- Study of cellular architectures and operations for data communications: to better understand the mechanisms in place in order to achieve the integration of the use of these cellular communications with transparency and independence by the VANET.

- Proposal and integration of a cellular communication alternative for the VANET multihoming architecture: to provide and implement a solution for using commercial cellular networks when deemed necessary by the VANET management services.

- Improvement of inter technology handovers taking into account cellular network connections: to adjust the system response and operations to the newly added communication option.

- Evaluation of the implemented solution: to evaluate the response and performance of the VANET on laboratory and real-world scenarios with the new cellular solution developed.

\subsection{Document Organization}

The remaining document is organized as follows:

- Chapter 2 - State Of The Art - This chapter presents a general analysis and description of fundamental concepts related to this dissertation: vehicular ad-hoc networks, network access technologies, cellular networks, mobility protocols and multihoming.

- Chapter 3 - Related Work - This chapter describes some of the works that better relate with the one presented in this dissertation.

- Chapter 4 - Mobility with Cellular Communications: Architecture - This chapter provides an overview of the VANET architecture, the challenges to overcome and the proposed architecture.

- Chapter 5 - Mobility with Cellular Communications: Implementation - This chapter presents, in detail, the implementation of the proposed architecture and supporting mechanisms. 
- Chapter 6 - Evaluation - This chapter describes the scenarios, the equipment and the platforms used in the performance evaluation. It presents and discusses the results obtained in the laboratory (controlled environment) and real-world scenarios.

- Chapter 7 - Conclusions and Future Work - This chapter provides conclusions on the fulfilment of the dissertation objectives, and also points to possible future improvements. 


\section{Chapter 2}

\section{State of the Art}

To better comprehend the work studied and developed in this dissertation, it is presented, in this chapter, the fundamental concepts and related aspects to the area of research. The chapter is structured as follows:

- Section 2.1 presents the concept and main characteristics of VANETs. It also discusses the different architectures, the main applications and also the challenges associated with this type of networks;

- Section 2.2 presents the leading wireless network access technologies used in VANETs. A overview of DSRC/WAVE, Wi-Fi and cellular technologies is also described;

- Section 2.3 presents the mobility protocol N-PMIPv6, which is the mobility protocol in use by the vehicular environment explored by this dissertation;

- Section 2.4 presents the multihoming solution used in the PMIPv6;

- finally, Section 2.5 enumerates the main ideas discussed in this chapter.

\subsection{Vehicular Ad-Hoc Networks}

The need either from the users or from the evolution of technology to force an always-on connection to the network allowing the exchange of data is a reality nowadays. In the vehicular environment where communications are needed for many purposes, therefore, requiring solutions to different problems, VANETs can be an answer to this requirement. The goal is to make possible communication via wireless technologies between $\mathrm{V} 2 \mathrm{~V}$ and/or between V2I or even Vehicle-to-Everything (V2X), to allow Intelligent Transportation System (ITS) capabilities [1], [2].

ITS V2I applications are made possible by the use of equipment, such as Road-Side Units (RSUs), that are placed along the roads. They are connected to the backbone network to allow the communication between the vehicles and the infrastructure. Additionally, an On-Board Unit (OBU) is placed inside the vehicle making the vehicle a mobile node of the vehicular network topology [3]. An example of a VANET is illustrated in Figure 2.1, where a vehicle connects to a RSU, and another vehicle connects to this vehicle via multi-hop communications. 


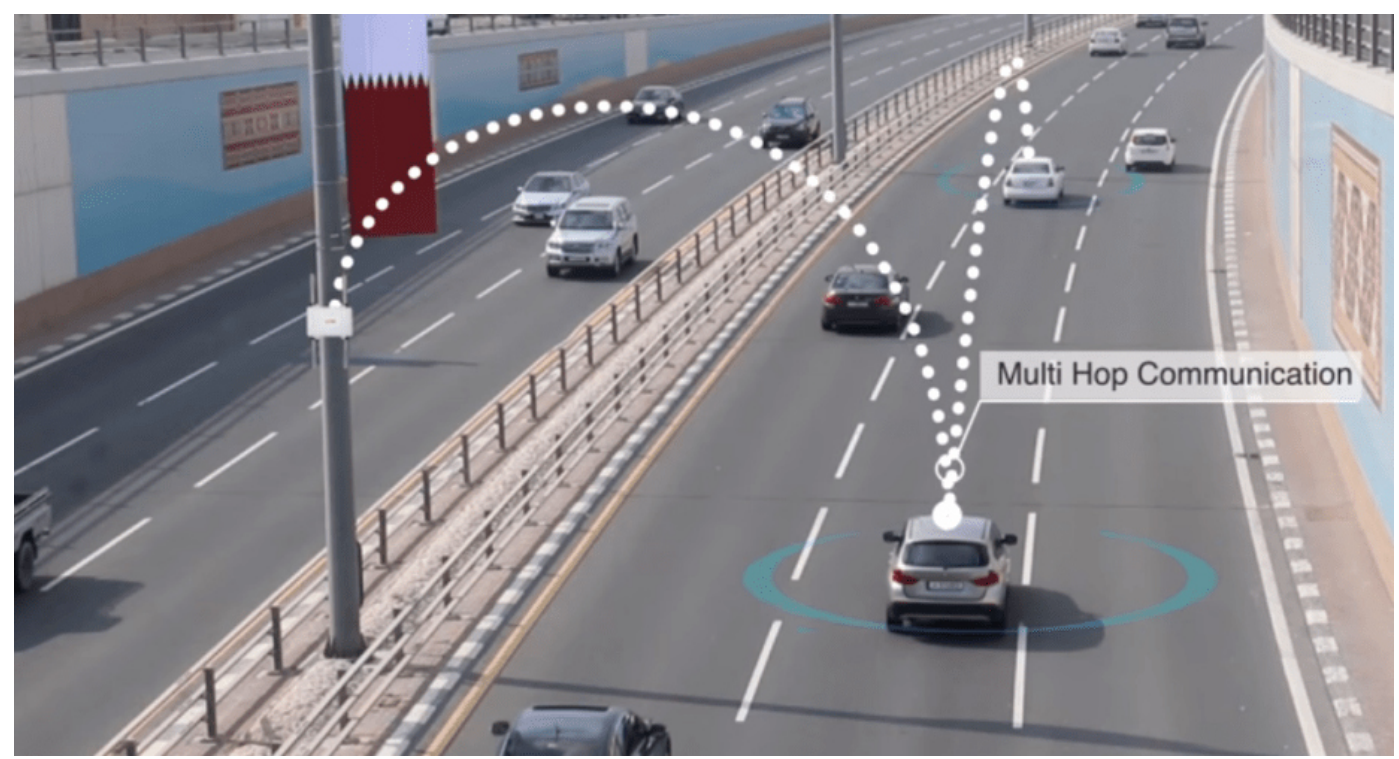

Figure 2.1: VANET example [4].

\subsubsection{Characteristics}

As a particular type of Mobile Ad-Hoc Network (MANET), VANET presents characteristics inherited but also new ones since it appears as a solution to overcome problems that MANETs were not prepared to deal with. In the next items, it is presented some of the main characteristics of this type of network [5], [6]:

- Scalability - The network will consist of vehicles equipped with OBUs, and since these represent the nodes connected to the network we can have as many nodes as the extent of the road area. The network needs to be able to accommodate this increase or decrease in the number of nodes, therefore it needs to be scalable.

- Predictability - Since the nodes are vehicles in a defined environment and allowing access to GPS information, it is possible to make predictions on the movements of these nodes.

- Dynamism - The network nodes are vehicles so this will imply that the network topology will be in constant change, and will need to be able to adapt to this dynamic environment. Aspects that will influence this dynamism will be the speed of the vehicles and their location.

- Processing Power - The processing power will be limited only by the equipment to be placed inside the vehicles. Nowadays, for the applications idealized for the nodes it is possible to say that there are no processing power limitations.

- Energy Power Constraints - Since the OBUs are placed inside the vehicles, they can be continuously powered by the vehicle battery. Therefore, we can consider the nonexistence of power constraints.

- Time Sensitive Information - One of the main objectives of ITS is to implement applications related to security, accident prevention, and vehicular traffic allowing this 
information to be exchanged between vehicles in a useful time window. This can be considered time-sensitive information, and therefore a VANET cannot compromise the delivery of these messages.

\subsubsection{Architecture}

One of the aspects in which a VANET differs from a MANET is that the nodes do not move in a completely random way. Instead, they move along roads allowing the positioning of fixed road-side equipment (RSUs). This will lead to VANET architectures being categorized into three types [7], [8]:

- Cellular/WLAN - The use of fixed cellular gateways or WLAN access points at the side of the road allows vehicles to communicate with the infrastructure (V2I), mainly for information and data gathering applications. Since wireless communication is made only between a vehicle and the fixed infrastructure, there is a need for full road coverage with RSUs.

- Ad hoc - Vehicles will rely on communications directly with other vehicles (V2V), without the use of fixed infrastructure along the road, mainly for the dissemination of information for safety, accident prevention, and security applications.

- Hybrid - This architecture takes advantage of both Cellular/WLAN and ad hoc architectures. It combines the use of communications between vehicles (V2V) and the communication between vehicles and the infrastructure (V2I), allowing a communication either in a single-hop or multi-hop fashion. This architecture increases the coverage area and does not require as much RSUs as a Cellular/WLAN architecture.

Illustrated in Figure 2.2 are examples of VANET architectures with the respective type of communications (V2V and V2I) between the nodes, also providing an illustration of the use of different access technologies.

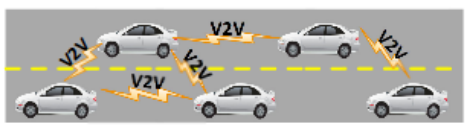

(a) Vehicle-to-Vehicle Ad Hoc Network

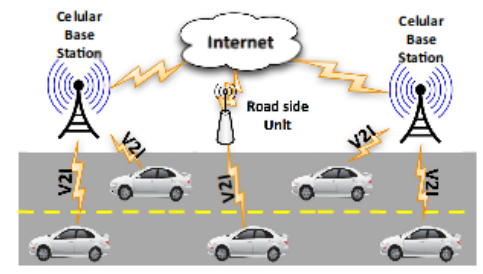

work

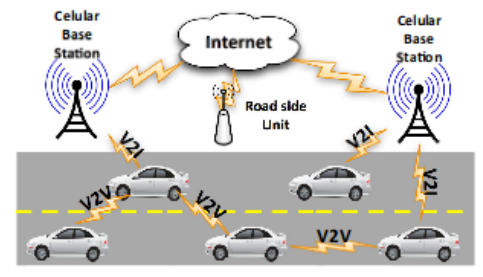

(c) Hybrid Architecture

Figure 2.2: Types of VANET Architectures [7].

\subsubsection{Applications}

A VANET can provide a way to allow the implementation of technological capabilities in a vehicle, and will provide a vast range of information that can be used for several types of applications. The range of such capabilities can go from the gathering of information about a traffic pattern, in order to improve an urban area efficiency in transit or for ecological aspects, 
up to optimize marketing strategies. The main types of applications to which VANETs are focused upon, nowadays, are [9], [10]:

- Safety Applications - This type of applications are the main booster for the development of VANETs. The main purpose is to help reduce the number of life-critical situations, like avoiding road accidents. Associated with this type of applications will be sensitive delay for the critical information to be exchanged between the nodes. Data like collision warning/avoidance, warning of sensitive zones (e.g. road work zones) and smart-driving vehicles assistance are all examples of information relevant to this type of applications.

- Comfort/Entertainment Applications - Providing ways to improve the user experience during the vehicular trip is the purpose of this type of applications. Allowing the connection to the Internet in order to access multimedia content, gathering information about the weather conditions or a point of interest (e.g. gas station), route planning to avoid traffic, tourist information, among others, are all examples of data for applications in this category.

\subsubsection{Challenges}

In the next items, it will be presented some of the challenges related to VANETs. These are problems that still need to be addressed and/or challenges [11] that still require further investigation and improvement so that this technology can continue to evolve.

- Security - The main concern nowadays in VANETs is the security associated with the network communications [12]. For example, attacks on the network that can disrupt the flux of critical information may put lives at risk. There is still a very strong need for research and innovative solutions in this specific aspect.

- Quality of Service - Another critical challenge for these networks is the quality of service. Users want more multimedia content and with better quality. Also, the number of applications requiring connection to the network is rising, which leads to an increase in the network traffic [13]. Avoiding network traffic overload problems and maintaining the availability of content flux and quality is a real growing issue.

- Resource Use - Solutions based on the use of several wireless access networks may already exist or be in development to avoid loss of signal but taking advantage of all the available resources in the boards in order to allow a transparent experience for the user is still a real need. For example, making use of a connection to the network via WAVE technology and changing to cellular technology when needed, without losing connection and having an optimized handle of these resources, either in upstream and downstream, will be the main focus of this dissertation.

\subsection{Network Access Technologies}

There are studies about several wireless network access technologies to be used in VANETs. However in this section, it will be presented the leading three access technologies adopted in general and more importantly adopted as solutions for the work in which this dissertation is based. 


\subsubsection{DSRC/WAVE}

DSRC were developed with the requirements of VANETs in mind, such as self-organizing, self-configuring, high mobility and dynamic topology [14]. DSRC uses a $75 \mathrm{MHz}$ spectrum in the $5.9 \mathrm{GHz}$ frequency band in the USA whereas in Europe and Japan uses a $30 \mathrm{MHz}$ spectrum in the $5.8 \mathrm{GHz}$ band. It is intended to provide communications V2V and V2I with support to data rates of up to $27 \mathrm{Mbps}$. The communication is made possible by the use of IEEE $802.11 p$ (WAVE). The terms DSRC and WAVE are used interchangeably.

\subsubsection{Wi-Fi}

IEEE 802.11 refers to a set of standards that define communications for Wireless Local Area Networks (WLANs). It is one of the most widely deployed and popular standards for wireless communications. One of the standards is IEEE $802.11 \mathrm{a}$, works at $5 \mathrm{GHz}$ and provides a data rate of $54 \mathrm{Mbps}$ to a range of at least 38 meters indoors and 140 meters outdoors. Another standard is IEEE $802.11 \mathrm{~g}$, providing the same data rate and coverage range but working at $2.4 \mathrm{GHz}[28]$.

For vehicular networks Wi-Fi presents some disadvantages that WAVE provides solutions for, such as low transmission range and bad performance with high-speed moving nodes. According to [15] it was concluded that the Packet Delivery Ratio (PDR) and throughput of IEEE 802.11p (WAVE) are higher than IEEE 802.11a (Wi-Fi) and that the average delay of IEEE 201.11p is lower than IEEE 802.11a, either in urban or highway scenarios.

Nevertheless, Wi-Fi can still be an option for communications in VANETs as it allows a wireless connection between the nodes and the infrastructure, but as an alternative to WAVE in the case of no connection available to this technology.

\subsubsection{Cellular}

A cellular network can be seen as a radio network distributed over land through cells where each cell includes a fixed location transceiver known as a Base Station (BS). Together these cells form a radio coverage over large geographical areas allowing the Mobile User (MU), using equipment such as mobile phones, to connect to this infrastructure so they can access the network, even when moving through cells during the communication (this capability of the network is known as handover). The concept of frequency reuse is used to increase the coverage area and also to allow multiple transmissions at the same time [14].

Giving some context on the origins and implementations of Cellular Networks, according to P. Gandotra et al. [16], M. Jamil et al. [17] and M. Mir et al. [18], it all started in the early 1980s with the First Generation (1G), and it consisted in circuit-switched. The Second Generation $(2 \mathrm{G})$ presented itself as the digital cellular in the late 1990's and, compared to the first, it provided services like email and Short Message System (SMS). With the 3G, in late 2000, came the support for data rates to up to 2 Mbps. Supporting services with improvements in voice quality and help in maintaining Quality of Service (QoS), enabling new innovative services like mobile videophone, video streaming, and Voice over IP (VoIP). The 4G, also known as Long Term Evolution (LTE), was developed with the aim to provide a system entirely based on IP, allowing higher speeds, broadband, and cheaper mobile services. Furthermore, the cellular network using $3 \mathrm{G}$ and $4 \mathrm{G}$ technologies through soft handoffs and seamless switching is able to support high mobility. 


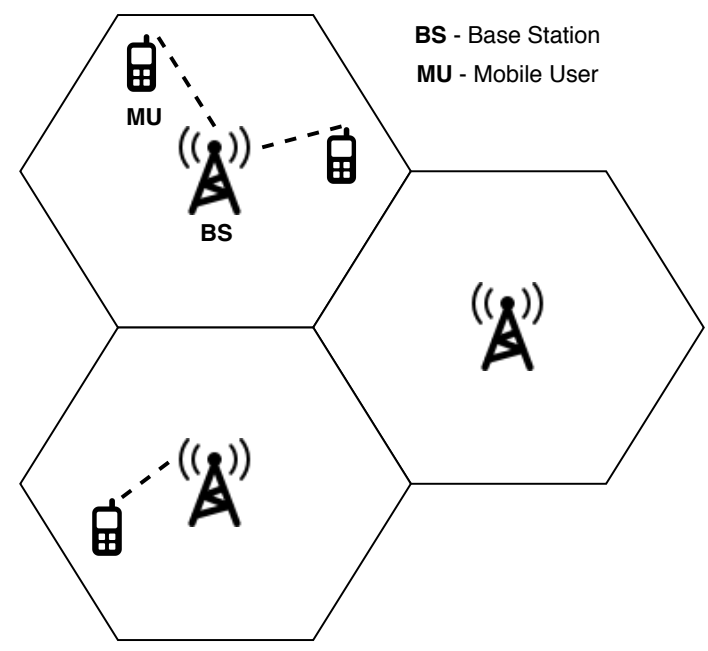

Figure 2.3: Cellular network representation.

Cellular networks provide (an almost) global coverage, making this an ideal wireless access network technology to avoid the challenge of losing connection due to bad network coverage. The $3 \mathrm{G}$ and $4 \mathrm{G}$ technologies have greatly improved the offer of high capacity and bandwidth communications, for example, for multimedia content. In Europe, Japan and the USA, many fleet and telematics projects already use different generations of cellular technologies. However, the drawback is the high cost and the higher latency rate that make these solutions less attractive for VANETs [19]. Nevertheless, the use of cellular technology presents a great feature, that being coverage, to take advantage of in a VANET.

\section{$2.3 \quad$ N-PMIPv6 Mobility Protocol}

When a node connects to a network it expects a steady and constant connection, whether this node is static or mobile. If it is a mobile node, there are different challenges presented to allow the connection to be persistent over time and anywhere (within the range of the signal to allow the connection to the network).

In a VANET the end-user will be connected to the vehicle that will represent a mobile node of that vehicular network. Vehicles are associated with high mobility, therefore, presenting an extremely dynamic environment for the network. When a device connects to the network it is defined an IP address, network mask, and default router so that the communication between nodes becomes possible. With static networks, this process happens when a node connects for the first time. If it disconnects and connects again through a different network it will gain a new configuration (IP address, network mask, and default router). This methodology will not be appropriated to a network that needs to support mobility, because it is required that a seamless communication between the vehicles and infrastructure does not fail. Since in the vehicular network users are connected to the vehicles that act as routers providing a sub-network inside the vehicle, when a vehicle changes its point of attachment to the network, the respective users will also change their point of attachment.

To support the entire network mobility, a mobility protocol is needed. A protocol responsible for the management of the location of the nodes and the management of the handoffs, fulfilling several requirements such as: Fast handover, since the nodes represent vehicles that 
are associated to an environment of high velocities and large number of cars; Continuity of the sessions of the users connected to the network even when their PoA changes; Transparency for the end-user that should not have concerns with any configuration beyond the OBU direct connection; Multi-hop that will allow the extension of the range of the network by allowing mobile nodes (OBUs) to connect to each other as a path to a fixed node (RSU).

Network-Proxy Mobile Internet Protocol version 6 (N-PMIPv6) is a mobility protocol originated from the merge between Proxy Mobile Internet Protocol version 6 (PMIPv6) [20] and NEtwork MObility (NEMO) [21], [22], in order to overcome the issues presented by each one. This N-PMIPv6 protocol is the mobility solution developed and currently in use by the vehicular environment explored by the Network Architectures and Protocols (NAP) research group. Following, in Figure 2.4 is illustrated a overview of this protocol.

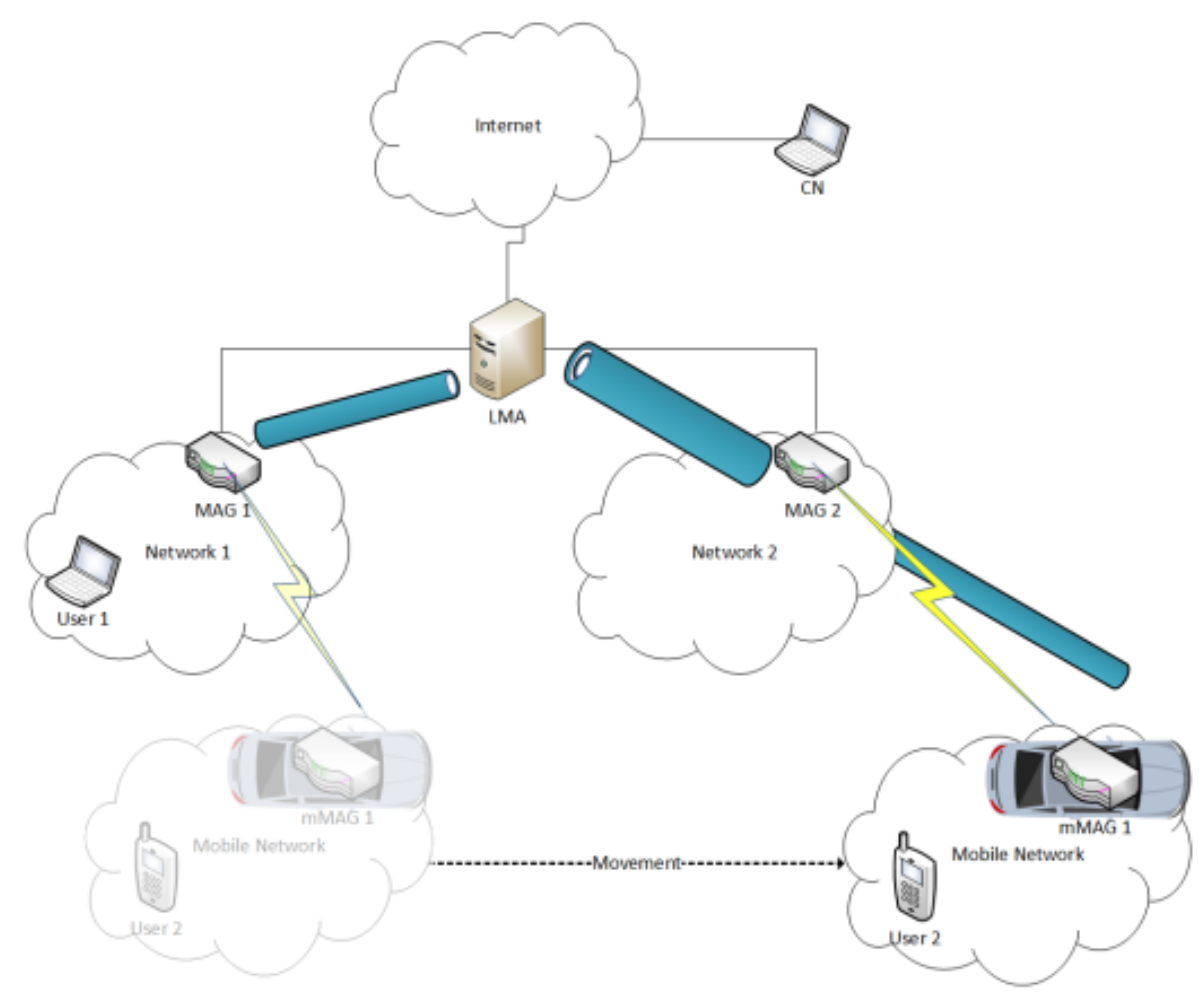

Figure 2.4: N-PMIPv6 protocol overview [23].

\subsubsection{Terminology}

As illustrated in Figure 2.4, to understand the N-PMIPv6 protocol operation it is first provided the respective terminology as follows:

- Local Mobility Anchor (LMA): This entity is the home agent for the mobile node, it is the anchor point for the mobile node's home prefix and manages the mobile node's binding state. 
- Mobile Access Gateway (MAG): It is responsible for performing the mobility related signalling on behalf of the mobile node that is attached to it, for tracking the mobile node's movements to and from its access link and to report to the respective LMA.

- Proxy Care-of Address (Proxy-CoA): Represents the address of the egress interface of the MAG and the endpoint of the IPv6 tunnel that will be used between the MAG and the LMA. The LMA views this address as the care-of address of the mobile node and registers it in the Binding Cache entry for the respective mobile node.

- Mobile Node's Home Network Prefix (MN-HNP): Is the prefix assigned to the link between the mobile node and the MAG by the LMA.

- Mobile Node IDentifier (MN-ID): Represents the unique identifier of the mobile node.

- Binding Cache Entry (BCE): Is responsible for storing the information related to every mobile node. Each entry contains the MN-ID, the MAG Proxy-CoA and the MN-HNP.

- Proxy Binding Update (PBU): It is the message sent by the MAG to the LMA for establishing a binding between the mobile node's home network prefix assigned to a given interface of the mobile node and its current Proxy-CoA.

- Proxy Binding Acknowledgment (PBA): It is the message sent by the LMA as a response to the $\mathrm{PBU}$ received. It also contains the prefix given to the mobile node.

- mobile MAG (mMAG): Is a MR that works very similar to the MAG in the PMIPv6 while being mobile.

\subsubsection{Operation Method}

An overview of the N-PMIPv6 protocol is presented in Figure 2.4. When a mMAG wants to join the network, it sends a Router Solicitation (RS) message to the serving MAG. When the MAG receives the message it sends a PBU message with the mMAG-ID to the LMA. The LMA, after receiving the PBU message, checks if there is a BCE that contains useful information such as the MN-ID, the MN-HNP, AR and the M flag (field that indicates if the message was sent by a MAG, $M=0$, or a mMAG, $M=1$ ), assigns the MN-HNP to the mMAG and creates its BCE. Also creates the IPv6 tunnel and sends a PBA message with the MN-HNP to the MAG. The MAG will forward the MN-HNP to the mMAG via a RA message allowing this way for the mMAG to configure its IPv6 address on the egress interface. When a user wants to join the network, a similar process occurs where the user sends a RS message to the mMAG and the mMAG sends the PBU message to the LMA that will fill the $\mathrm{BCE}$ such as the previous case but this time with the M flag defined as 1 (due to the PBU being sent by a mMAG) and create the IPv6 tunnel with the mMAG as endpoint sending the PBA with the MN-HNP attributed to the user. The mMAG after receiving the PBA creates its endpoint of the IPv6 tunnel and sends the user a RA with the assigned MN-HNP.

The N-PMIPv6 protocol is able to support the host and the network mobility, and there is no required modification or extension to the MN. Also, in an handover scenario the MAG 
informs the LMA about the mMAG movement and not about every single user connected to the mMAG. Due to these characteristics, this mobility protocol proved itself as a coherent choice to satisfy the VANET needs, and is still serving as the mobility protocol for the VANET project and consequently for the work done in this dissertation.

\subsection{Multihoming}

Nowadays, it is possible to have access to multiple wireless technologies, giving the user the option to connect its mobile devices to a number of different access networks, if available. Therefore the use of only one of these access networks instead of several simultaneously will not provide the full advantage of all available resources for the user.

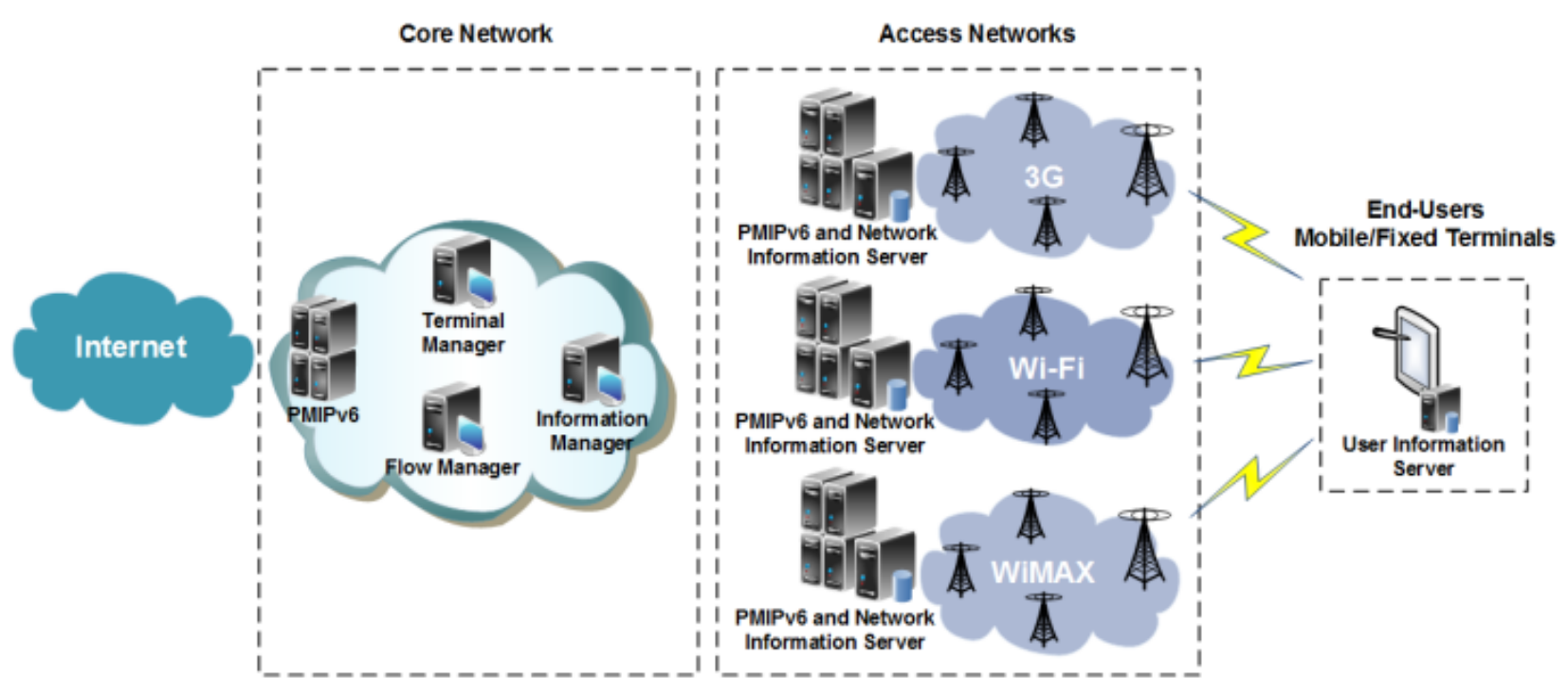

Figure 2.5: Multihoming Architecture Example [24].

Several research works have presented solutions for the real application of multihoming capabilities providing conclusions about the reliability, performance and advantages of such practice [25], [26], [27].

For the VANET where this dissertation is focused upon, the main purposes that multihoming may bring are: the capability of performing Load Sharing, allowing a higher throughput by aggregating the throughput of each individual connection; to increase the reliability, for having multiple connection paths to the network; to increase the QoS for the user by having load balancing capabilities; and to best use and manage all available resources, possibly allowing a decrease in the economic costs [28]. Next, it will be presented the multihoming protocol inherited from the base work of this dissertation.

\subsubsection{Proxy multihoming extension for PMIPv6}

The multihoming architecture [29], [30] used in this project was developed by the NAP research group and has the objective of taking advantage of all access networks available in order to increase the overall network performance. It was developed as an extension to the PMIPv6 protocol where the mobility is therefore assured by the PMIPv6 protocol, and the 
multihoming management is assured by a set of new entities that were added to the LMA and to the MAGs. The extension is based on a proxy-server placed in the network and, to achieve multihoming, a single OBU can have multiple paths using IP replication. This aspect, and the fact that this solution is implemented over the network layer, allows the end-user to take advantage of it without the need of any additional requirement.

\subsection{Final Considerations}

This chapter provided an introduction to vehicular networks, to the respective leading network access technologies used in VANETs, given an overview to cellular networks, and also to the mobility protocol and multihoming approach of the VANET developed by the NAP research group ${ }^{1}$.

Taking into account the challenges that are associated with VANETs, mainly the high mobility of the nodes, and therefore, the consequent high number of handovers and the growing number of vehicles endued with network access capabilities, selected mobility protocols were studied, in particular the N-PMIPv6, and multihoming in order to better understand these vehicular architectures. It were also presented the leading access network technologies that are used in this type of systems to understand their differences.

This chapter aims to allow the reader to better understand the technologies and scenarios of the state of the art of vehicular mobility. After this introductory chapter, it will be presented some related work, where it will be discussed several research works that focus in the area of interest for this dissertation.

\footnotetext{
${ }^{1}$ https://it.pt/Groups/Index/62
} 


\section{Chapter 3}

\section{Related Work}

When working on a project, it is always required to have an understanding of the work being developed, or already presented, about the respective topics. For the development of VANETs, a significant effort is being made by numerous academic and corporate entities around the world.

This chapter provides a discussion on some of the main topics related to the work of this dissertation, and is structured as follows:

- Section 3.1 presents an overview of several topics related to the technologies and architectures used in VANETs, also providing an overview of the interworking of DSRC and cellular;

- Section 3.2 provides an overview of some of the works related to the use of $5 \mathrm{G}$ technologies in VANETs;

- Section 3.3 presents some insights about the use of cellular communications in VANETs and the main ideas discussed in this chapter.

\subsection{VANET Technologies and Architectures}

The three leading technologies that serve as the base for the majority of the studies on VANETs are, as previously mentioned, WAVE (802.11p standard), Wi-Fi (IEEE 802.11a/b/g standards) and 3G or 4G (LTE) cellular (Cellular-Vehicular to Everything - C-V2X is also gaining momentum in a $5 \mathrm{G}$ environment).

According to Alexey Vinel et al. [31], which compared LTE against WAVE to evaluate the preferable one to support cooperative vehicular safety applications, concluded that the abilities of LTE to support beaconing for vehicular safety applications are poor, because the network easily becomes overloaded. Furthermore, for this purpose, the use of cellular access involves additional costs compared to WAVE. Therefore, ad-hoc WAVE architectures are more promising for vehicular safety.

Chen et al. [32] proposed a VANET solution using LTE only, presenting a V2X architecture based on this access technology with several adaptations: a systematic and integrated V2X solution based on LTE time-division, called LTE-V. They presented simulations conducted to verify the performance of LTE-V, concluding that LTE-V needs to encompass new features 
to meet the demands of $\mathrm{V} 2 \mathrm{~V}$ communications, such as congestion control and heavy density traffic, low-cost broadcasting and simplified OBU and RSU device design.

Figure 3.1 illustrates examples of communications in a VANET with integration of cellular networks, as discussed in [33]. The work in [33] provides a survey on data offloading techniques for VANETs, discussing that the generated mobile network traffic has been rapidly increasing to the point of becoming a big burden on the current cellular networks, and therefore VANETs with WAVE and Wi-Fi capabilities allow for a divided burden among different technologies, avoiding an overload of a specific network connection.

The work in [34] advocates the use of LTE alongside WAVE as a valuable addition, allowing the use of each technology to the more appropriated applications based in each technology performance. Also, according to Mikko Valta et al. [35], WAVE has a very good performance in terms of delay and line-of-sight range, and LTE could be an interesting solution as a complement mainly due to the range which is higher than WAVE.

In this dissertation, implementing the use of cellular communications alongside WAVE and Wi-Fi for V2I communications is seen as the best way to increase the VANET capabilities, and without requiring big changes to its architecture as it is expected.

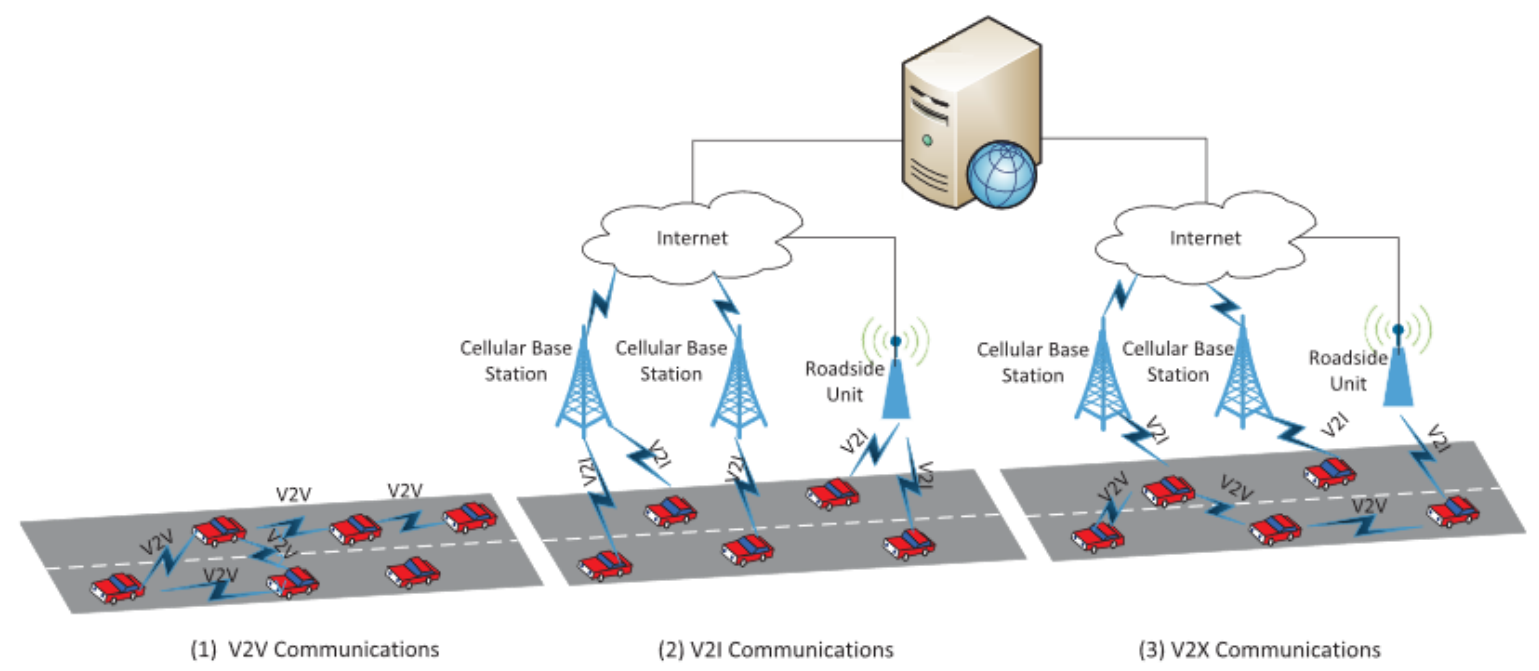

Figure 3.1: Illustration of communications in VANETs with integration of cellular networks [33].

Some previous research focused in cellular-VANET heterogeneous networks where WAVE is used for V2V communications, and cellular is used for V2I communications. Following, it is presented the works that use this methodology.

The work in [36] presented the integration of a WAVE VANET with 3G networks by using mobile gateways (some of the vehicles). The management of the network is done using WAVEbased multi-hop data transfers between vehicles, and with a designated gateway, the vehicles transmit the VANET data via $3 \mathrm{G}$ to the infrastructure.

The work in [37] proposed a hybrid architecture where V2V communications use WAVE and V2I communications use LTE. The result is a so-called Cloud-VANET that provides low network overhead with high mobility management, and high coverage. Simulation results show an improved overall network stability and performance. 
In [38] it was proposed an efficient QoS-based gateway selection algorithm for VANETs to LTE advanced hybrid cellular network. Simulation results showed that the algorithm makes efficient decisions for electing the appropriate gateway with the best VANET and infrastructure features, and the criterion for such decision includes traffic load, received signal strength and link connectivity duration.

Seyhan Ucar et al. [39] presented a hybrid architecture (VMaSC-LTE) that combines the use of WAVE and LTE to achieve a high data packet delivery ratio and low delay, while keeping the use of cellular at a minimum level. They proposed a Vehicular Multi-hop algorithm for Stable Clustering (VMaSC) that is responsible for providing V2V communications via WAVE and V2I communications via LTE.

Presenting a hybrid architecture where it is used WAVE for V2V communications and cellular for V2I communications, such as discussed in the works above, goes in a direction different from the goal for this dissertation where the use of WAVE, Wi-Fi and, now envisioned, cellular may be used for V2I communications and only WAVE is used for V2V communications.

The use of the cellular technology alongside WAVE is not an original concept. In [40] the authors attempted to improve the Internet access, having more than one access network, being WAVE and $3 \mathrm{G} / 4 \mathrm{G}$. Their focus was on the performance of the handover process between the two technologies, providing an optimized handover decision algorithm. For the interest of this dissertation, their work focused mainly in the analytical model and respective simulations, and did not provided relevant insights of the architecture and respective implementation.

Marco Gramaglia et al. [41] proposed a solution for providing Internet connectivity in multi-hop VANETs, with vehicles using cellular networks to assure always-on connectivity but also opportunistically offloading non-critical flows to the multi-hop wireless local area network (WLAN). Simulated results showed that the cellular network can be offloaded by a factor of up to $80 \%$. Their work focused on proposing the framework based on the idea of vehicles equipped with $3 \mathrm{G}$ and WLAN, and developing a simulator to obtain conclusions on the performance of their solution (SILVIO), which builds upon a set of other solutions adapted to the vehicular environment.

A multi-technology seamless handover mechanism for vehicular networks that integrates extended mobility protocols based on MIPv6 and PMIPv6 was presented in [42]. Validation and evaluation results of the proposed handover approaches were provided, combining three technologies: WAVE, Wi-Fi and 3G. The integration of cellular communications used a dedicated infrastructure to connect the mobile node using $3 \mathrm{G}$ to the VANET (similar to a 3G RSU) as presented in [43].

In [44] the authors proposed an integrated wireless networking system composed by a mobile ad hoc network, a cellular network and also a satellite network, in order to reduce the network deployment cost, to support more terminal types and to provide mobile users with better maritime services. This work was in the scope of maritime communications and not urban VANET communications.

According to Khadige Abboud et al. [45] and illustrated in Figure 3.2, it is shown an example of V2X communications with a DSRC-cellular hybrid approach in a urban scenario. They provided a survey on potential DSRC and cellular network technologies for efficient V2X communications, and concluded that several technical issues, mainly originating in the highly dynamic topology of VANETs, need to be resolved and, for that reason, there is a need for research and development on platforms that integrate DSRC and cellular networks. 


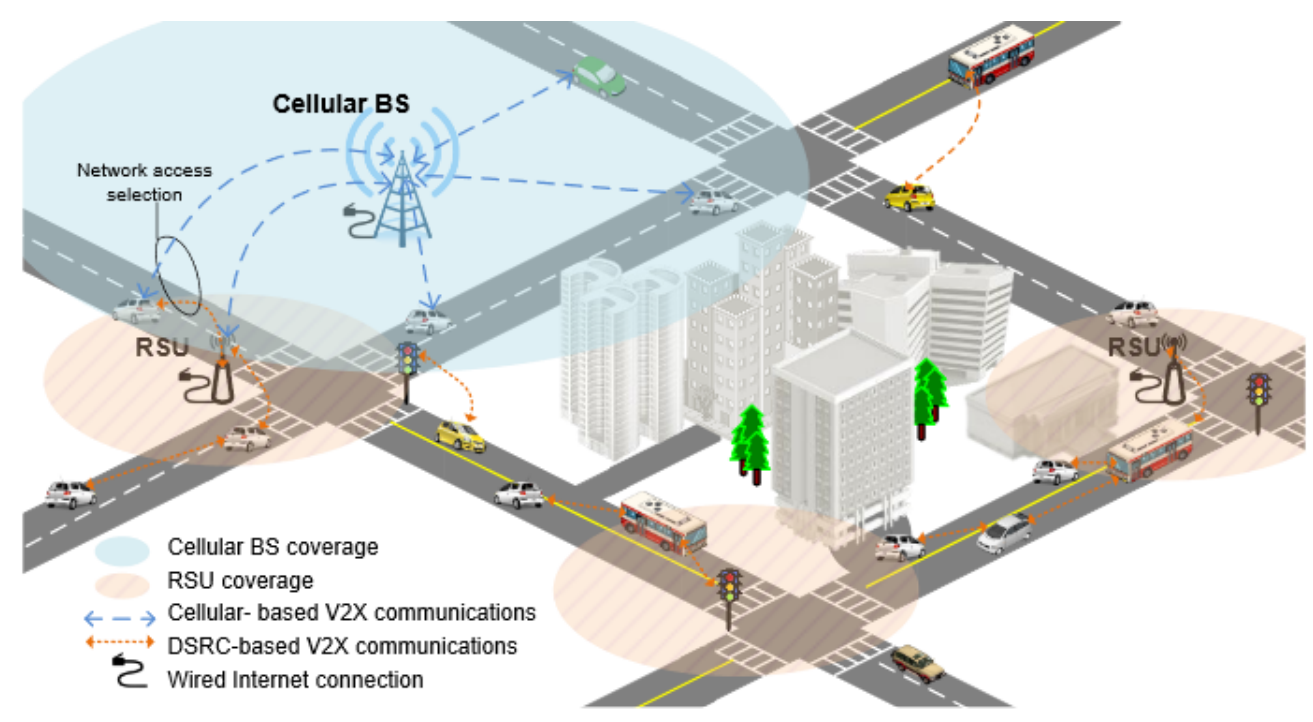

Figure 3.2: V2X communications in a DSRC-cellular hybrid urban scenario [45].

We can find different architectures using the cellular technology in VANETs such as: the use of $3 \mathrm{G}$ or LTE for all V2X communications; or the use of cellular technology for V2I communications maintaining the use of WAVE for V2V communications; or the interworking of DSRC and cellular technology for V2X. Nevertheless, in this dissertation, the intended approach is to maintain WAVE and Wi-Fi V2I communications, and alongside have the possibility to use the cellular technology only for V2I communications as a last-resort, when no other connection is available.

\subsection{G for Vehicular Environments}

During the research, several articles were found, related to the next steps for cellular technologies and VANETs that are considered useful to provide some insights in the current trend of the works being developed in order to understand the focus of cellular technologies and their relation with future VANETs. Thus, this section aims to provide a picture of the current and future trends of this area.

The Fifth Generation of cellular communications is approaching fast, with great research and development efforts by the academy and industry [46] and that is expected to bring a new era in mobile communications [47]. Idealized to allow access through different radio technologies at the same time by the terminal, and combine these flows to optimize resources and performance, the primary goal is to achieve higher data rates than previous generations, to fulfil the increasing demand of subscribers and the increasing amount of applications and services.

Several architectures have been proposed, and performance tests have already been conducted. For example, the work in [48] proposes a new VANET architecture that integrates the centralization and flexibility of Software Defined Networking (SDN) and Cloud-RAN (C-RAN) with $5 \mathrm{G}$ communication technologies. They also make use of a proposed fog computing framework (comprised of zones and clusters) at the edge, to avoid the frequent handovers between the vehicles and the RSUs. They present the comparison with other architectures of the trans- 
mission delay, throughput and control overhead on the controller, and results to support the improvement of such parameters with their system.

Another work [49] highlights the fact that fog computing in $5 \mathrm{G}$ proved itself to be a solution for faster processing in delay sensitive applications, such as VANETs, and it proposed a three-way integration between VANETs, SDN and 5G aiming for a resilient VANET security design approach, striking a good balance between network, mobility, performance and security features. It also shows how such approach can secure VANETs from different types of attacks, such as DDoS.

The interworking of VANET technologies and cellular technologies is seen as the next step to allow the exponential increase of functionalities a VANET can provide. In this dissertation, by having the intention of integrating a cellular solution that allows the use of commercial cellular providers without requiring contributions by these entities or the users, it is possible to pave the way for the use of $5 \mathrm{G}$ technologies in this VANET.

\subsection{Final Considerations}

Several works using hybrid VANET architectures with WAVE and cellular technology focus on proposals of algorithms for traffic offload. These works usually come across VANETs that form a cluster of vehicles communicating via WAVE (V2V), and use cellular technology (3G or $4 \mathrm{G}$ ) to communicate with the infrastructure (V2I).

This dissertation aims to improve a VANET that already works with WAVE and Wi-Fi, with a solution for exploring commercial cellular networks as a new and complementary V2I communication link. With these several studies, it is possible to justify that an implementation of a cellular solution in the VANET proves itself as a valuable resource.

Researches related to different VANET aspects were discussed, providing a base knowledge of the current reality in research. From the use of cellular technologies as a choice for VANETs to have a V2X communication, or just V2I communications exclusively, it were taken conclusions about their significance related to this dissertation. 


\section{Chapter 4}

\section{Mobility with Cellular Communications: Architecture}

The main objective of this dissertation is to present a solution based in cellular communications to be used efficiently as a backup alternative for a VANET's V2I communication. For this approach, we considered the use of commercial cellular data services. This chapter presents the following topics:

- Section 4.1 provides an overview of the architecture and operation of the VANET explored in this work;

- Section 4.2 presents the challenges and the proposed architecture for the integration of the cellular technology in the VANET's V2I communication alternatives.

\subsection{Current Architecture}

The work developed in this dissertation comes as an addition to an already functional VANET. This VANET offers mobility and multihoming features for V2I links via different wireless communication technologies, such as WAVE and Wi-Fi. Furthermore, it allows terminals connected to OBUs to establish Internet sessions and keep them active when in movement.

The VANET, as presented in [50], [23], [24] and [51], implements the following features:

- Support of full network mobility for the vehicles (their OBU's) and their users;

- Support of IPv4 Internet connectivity for users attached to OBUs;

- Integrated V2I multihoming capabilities, based on WAVE and Wi-Fi, with network performance maximization;

- Support of multiple V2I connections to WAVE Point-of-Attachments (PoAs);

- Support of V2I connections to Wi-Fi PoAs;

- Technology selection based on RSSI. 


\subsubsection{Mobility Protocol}

The N-PMIPv6 protocol is the current mobility solution in use by the target VANET. It is based on the PMIPv6 solution in [50], [23], [24] and [51], being managed by three main entities: the Local Mobility Anchor (LMA), the Mobile Access Gateway (MAG) (corresponding to RSUs) and the mobile MAG (mMAG) (corresponding to OBUs). These entities cooperate in order to allow user terminals to connect to the Internet through OBUs. In this section it is explained the operation of each entity to provide a better understanding of this mobility protocol.

\subsubsection{LMA Operation}

In this protocol, the users can connect to the mMAGs or to the MAGs. No matter if the users are connected to a sub-network of the mMAGs, the LMA will only register the mMAGs, which means that mMAGs will be seen as users connected to the network. The LMA is responsible for the management of the mobility process and the registration of the location of each mMAG belonging to the VANET. For that it uses a BCE.

The LMA operation is based on a finite state machine illustrated in Figure 4.1. When an mMAG connects to a MAG this last entity will send a Proxy Binding Update (PBU) message to inform and register the node on the LMA. The LMA will receive and process the PBU message sent by the MAGs in the following ways:

- New mMAG: if the mMAG that triggered the registration does not have a BCE, the LMA will register the new mMAG. The LMA will fill the new mMAG's BCE according to the information received on the PBU message.

- Already registered mMAG: if the mMAG that triggered the registration already has a BCE, the LMA will update it with the information received on the PBU message.

When a new mMAG is registered in the LMA a new entry is added in the BCE, if there is no IPv6 tunnel between the MAG and the LMA, it is created, and the required routes to the mMAG are configured. Finalizing the registration of the mMAG, a PBA message is sent to the MAG to confirm the completion of the registration process.

When the PBU received by the LMA refers to a known mMAG, the LMA checks if the PBU lifetime as expired. If not, the LMA checks if the PBU is from the MAG identified in the BCE, and in case of confirmation, it will only refresh the BCE entry and send a PBA to the MAG. Otherwise, an handover process occurs between the old IPv6 tunnel, from the LMA to the previous serving MAG, to the new IPv6 tunnel between the LMA and the current serving MAG. If the number of mMAGs connected to this MAG is zero it will delete the IPv6 tunnel to the MAG and respective routes, and then register the mMAG as a new mMAG. If the lifetime as indeed expired, the LMA will delete the mMAG's BCE and the tunnel to the MAG if there are no more mMAGs registered in this MAG. The LMA will then send a PBA to the MAG to confirm the elimination of the mMAG's entry, finalizing this process.

\subsubsection{MAG/mMAG Operation}

The MAG entity is responsible for the detection of movement of mMAGs and their users, and to communicate their location to the LMA. The mMAG entity is in reality a normal MAG but with mobility capabilities (a mobile node), while the MAG corresponds to a static node. 


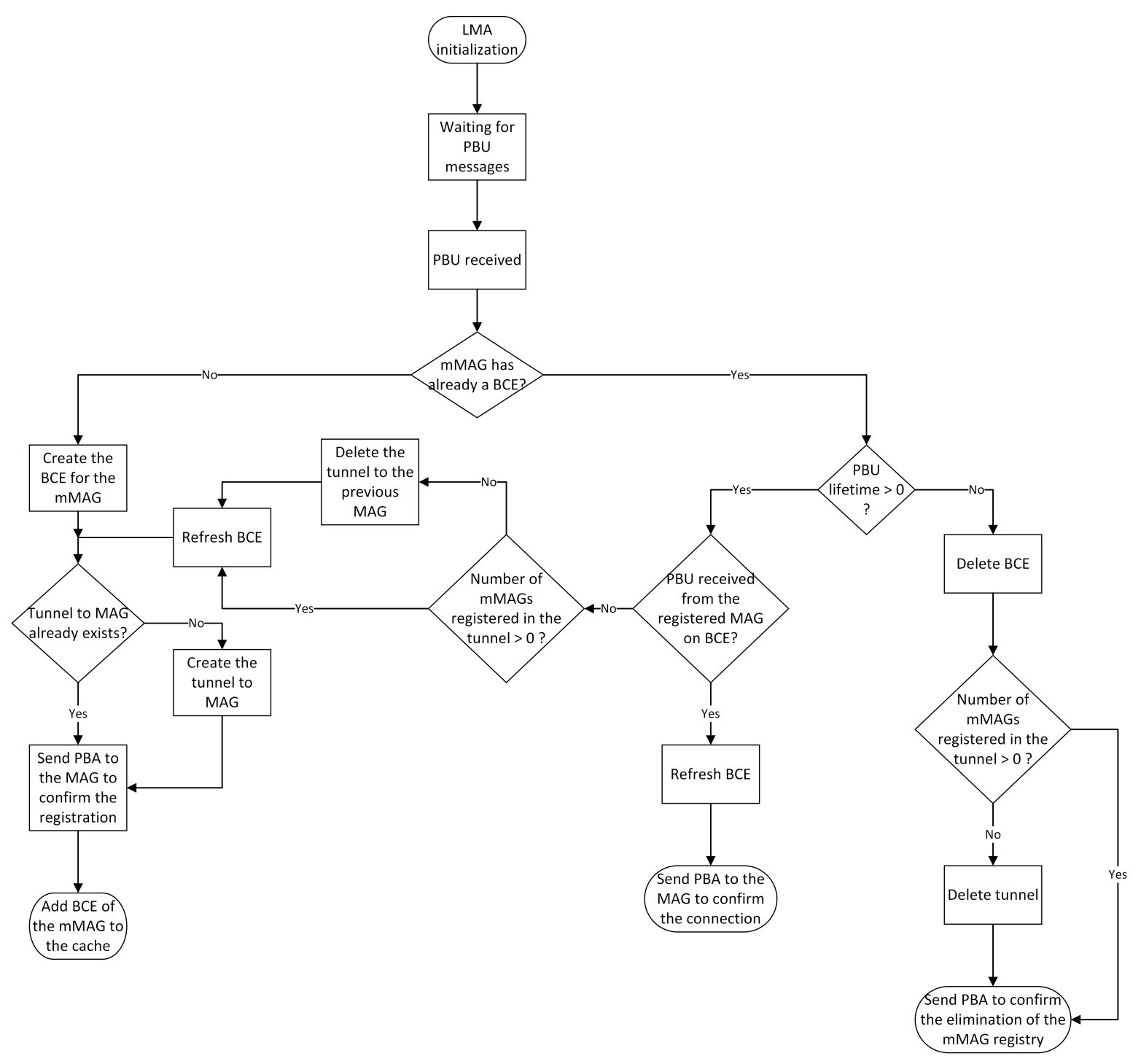

Figure 4.1: LMA operation flow diagram [50].

For this, the operation of both entities is quite similar and it is part of the MAG functionalities to decide if it has to adopt a MAG (also called RSU) or mMAG (also called OBU) behaviour (as referred, depending if it will be a mobile MAG or a static MAG).

The MAG and mMAG operations are based on a finite state machine, represented in Figure 4.3. And they can be divided into two main parts:

- Deciding if it is a MAG or a mMAG: Based on a configuration file. If the predefined Egress Address is present in the configuration file it will run as a MAG entity, if it is not present it will run as a mMAG entity.

- Capture of packets: If it is a MAG, it will capture RS and PBA messages; if it is an mMAG, it will also capture Router Advertisement (RA) messages. 
If an mMAG receives an RA message, this means that it has successfully joined the VANET, and fetch from that RA the IPv6 network prefix to configure the interface address. If an RS message is received, this means that another mobile node is trying to join the network, and in the case that the mMAG is the real destination of this RS message, the mobile node that sent the RS will be processed in order to join the VANET via multi-hop communications.

Based on the principle that MAG and mMAG have a very similar process and therefore from the point-of-view of the LMA both entities are identical, taking into consideration that mMAGs always obtain a valid route ensuring the connectivity for the users, these routes and tunnels will allow the mobile node to be reachable by the LMA or the MAG and between the mobile node and the LMA is only network routing. As shown in figure 4.2, the mMAG2 is seen as a normal user to the mMAG1 and it will connect and register on the network following the normal procedure as if it were connecting through a MAG to the LMA. Normal connectivity to the network is provided by the mMAG2.

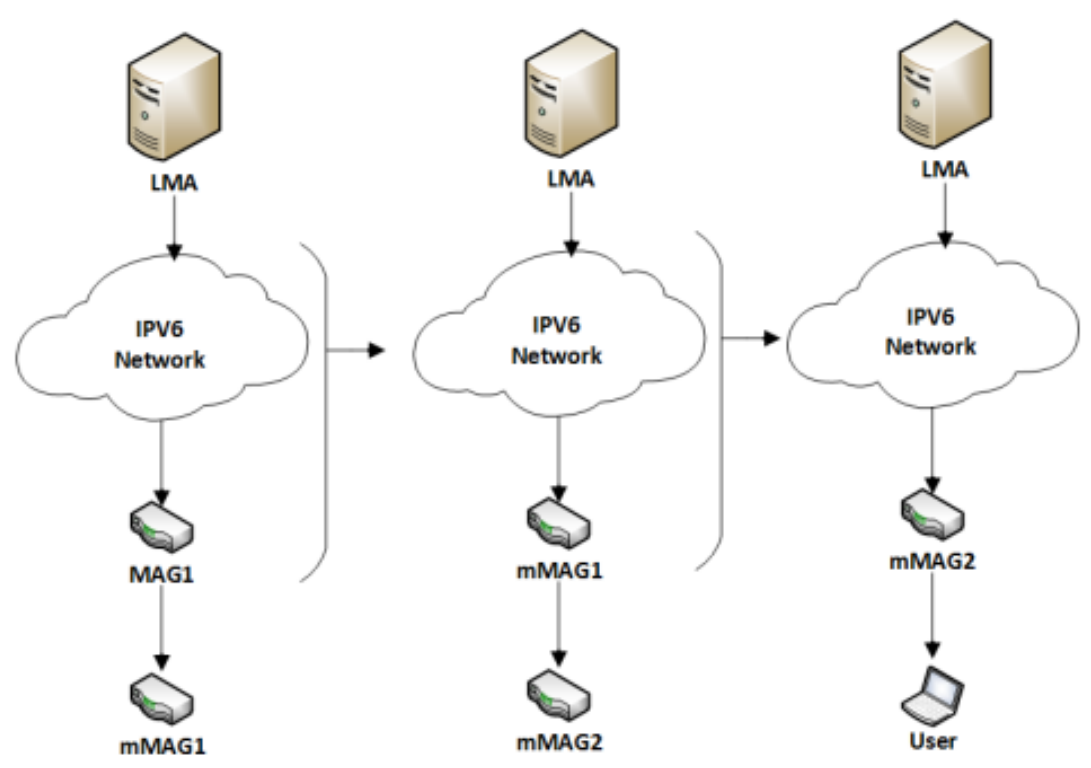

Figure 4.2: N-PMIPv6 Network Abstraction representation based on [23]

When an mMAG is detected, if it already has a BCE entry two scenarios are presented: the $\mathrm{BCE}$ is definitive and the RS was sent to refresh the BCE maintaining the session, or if the BCE was temporary it will become definitive followed by the creation of the IPv6 tunnel to the LMA. In the case of the mMAG not having an associated BCE, a temporary entry will be created with the intent of the LMA to send a PBA for finalizing the registration process and create the definitive $\mathrm{BCE}$.

When the MAG entity is operating as itself, without mobile characteristics and therefore as a RSU, the process of capturing RA messages is not needed and are only processed RS and PBA messages. 


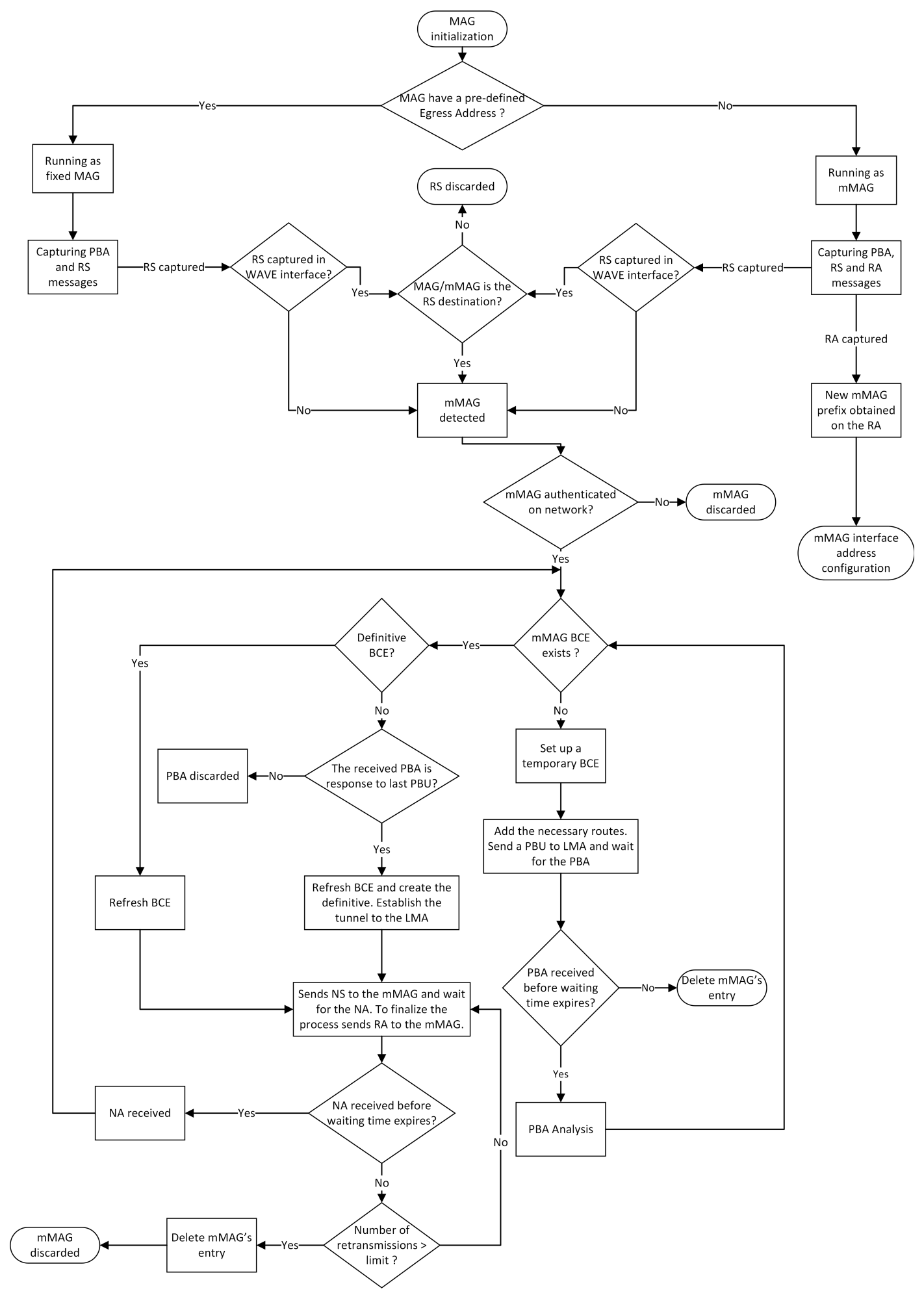

Figure 4.3: MAG and mMAG operation flow diagram [50]. 


\subsubsection{User Internet IPv4 connection support through OBUs}

Since this protocol, N-PMIPv6, only provides IPv6 support, in order to provide IPv4 Internet access to the end-user connected to an OBU, a solution based on IPv4-in-IPv6 tunnelling, connecting the LMA directly to the mMAG, as been previously proposed in [50] and [23], as illustrated in Figure 4.4. With a Network Address Translation (NAT) box placed on the LMA, it is possible to convert the OBU users' requests into LMA requests to the Internet, hiding the network below the LMA under a unique IPv4 address from the Internet point-of-view. When the LMA receives packets from the Internet, the NAT and the LMA redirect them to the respective sender (user connected to the OBU that initiated the communication) through the established tunnels. The OBU provides a IPv4 network for the end-users, presenting itself as a Wi-Fi PoA.

Illustrated in Figure 4.4 is a user terminal connected to the VANET and with IPv4 Internet access, where the serving OBU is connected via Wi-Fi and WAVE RSUs simultaneously, and therefore it has two IPv4-over-IPv6 tunnels, one for the Wi-Fi connection and another for the WAVE connection, providing multihoming capabilities. The LMA provides IPv4 Internet access to the user terminal through both these tunnels, and is responsible for the NAT process. The IPv6 tunnels between the RSUs and the LMA correspond to the normal procedure explained in the previous section to allow VANET related communications.

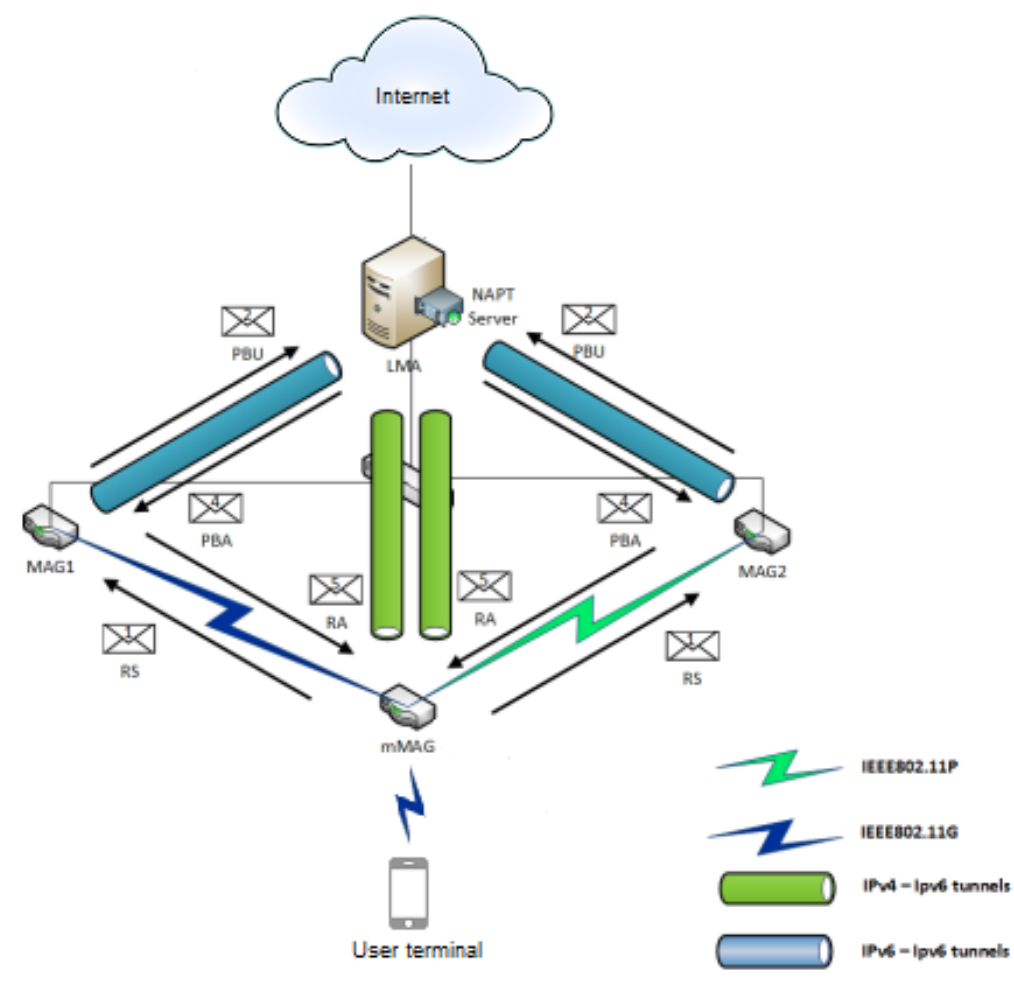

Figure 4.4: Example of user Internet access through IPv4 over IPv6 tunnels with multihoming (adapted from [23]). 


\subsubsection{Connection Manager Operations}

Providing an overview discussion of the connection manager that is executed in the mMAGs (also known as OBUs), it allows the mMAG to connect to MAGs with different technologies at the same time, taking advantage of both WAVE and Wi-Fi links providing an increase in performance and availability to the network. It also allows to connect to the Wi-Fi MAG that presents the highest RSSI, due to the OBU only having one Wi-Fi interface it will only be possible the connection to one Wi-Fi MAG and for this, the selection criteria is to connect to the Wi-Fi network that provides the best RSSI. Also allows the connection to several WAVE MAGs, being possible because the WAVE technology does not require an association process and therefore the connection manager will connect to all WAVE networks that satisfy a minimum RSSI threshold. The connection manager will perform the necessary interface configurations and manage the uplink/downlink routes. It is composed by three main threads: the WAVE thread; the Wi-Fi thread; and a third thread that manages the reception of RA messages.

\subsubsection{Considerations}

In summary, this mobility protocol takes advantages of the available connections via WAVE and Wi-Fi provided by MAGs (RSUs) for mMAGs (OBUs) to connect to, having multi-hop and multihoming capabilities. Furthermore, it supports access to the Internet for end-users via IPv4.

An aspect that will need to be later addressed is that, contrary to the N-PMIPv6, that works with IPv6, the cellular network works in IPv4. In the next Section, it will be presented an architecture to integrate cellular communications alongside the existing methods, redirecting network traffic via a commercial cellular network when the VANET cannot grant V2I connectivity with Wi-Fi or WAVE.

\subsection{A Multi-technology VANET Architecture}

VANETs are associated with high mobility, and usually, a great number of mobile nodes where it cannot be expected a full control over their behaviour. Such mobile nodes are capable of free movement over long distances, bringing the possibility of scenarios that, even with a wide deployment of WAVE or Wi-Fi PoAs, it is not possible to guarantee a continuous V2I connectivity with these technologies.

In order to avoid the downtimes that may occur, that contemplate, for example, unpredictable scenarios associated to the location of the mobile nodes or even the failure of infrastructure equipment, it was designed a solution to explore cellular networks as a backup V2I link.

\subsubsection{Overview of the Architecture}

The proposed approach will make use of WAVE and/or Wi-Fi whenever possible, and if no connection to these technologies is available within the desired and acceptable parameters, the system will resort to cellular networks, in order to maintain the full VANET connectivity, allowing the end-users to access the Internet. 
To take the most out of cellular networks, it is proposed an approach that allows the use of any commercial cellular provider without the need of collaboration from these entities. In other words, we will consider a commercial cellular network as a non-cooperative network infrastructure for our solution. This way, we can use already existing cellular infrastructures, instead of requiring the investment and resource allocation for the deployment of a private cellular network.

The main goal of this dissertation is to improve the connectivity of a VANET by making use of a commercial cellular network provider as a backup V2I link provider. This should allow the VANET to maintain connections between the LMA and mobile nodes (OBUs), and provide Internet access to the users via an OBU even when it loses connection to WAVE and Wi-Fi RSUs. Cellular V2I links are to be abandoned as soon as OBUs restore their VANET connection through a WAVE or Wi-Fi RSU. Through the use of a cellular V2I link, we aim to drastically reduce the number of V2I dead zones for mobile nodes and, in the meantime, reduce the need for extra equipment (such as more WAVE or Wi-Fi RSUs).

The proposed architecture illustrated in Figure 4.5 presents a combination of the VANET capabilities with the addition of the advantages of cellular technologies. As shown in Figure 4.5, when the OBU is connected to the VANET via cellular, this connection is made possible, not by connecting to a RSU, but by connecting to the cellular network through a respective BS. For that, each OBU will be equipped with a cellular interface.

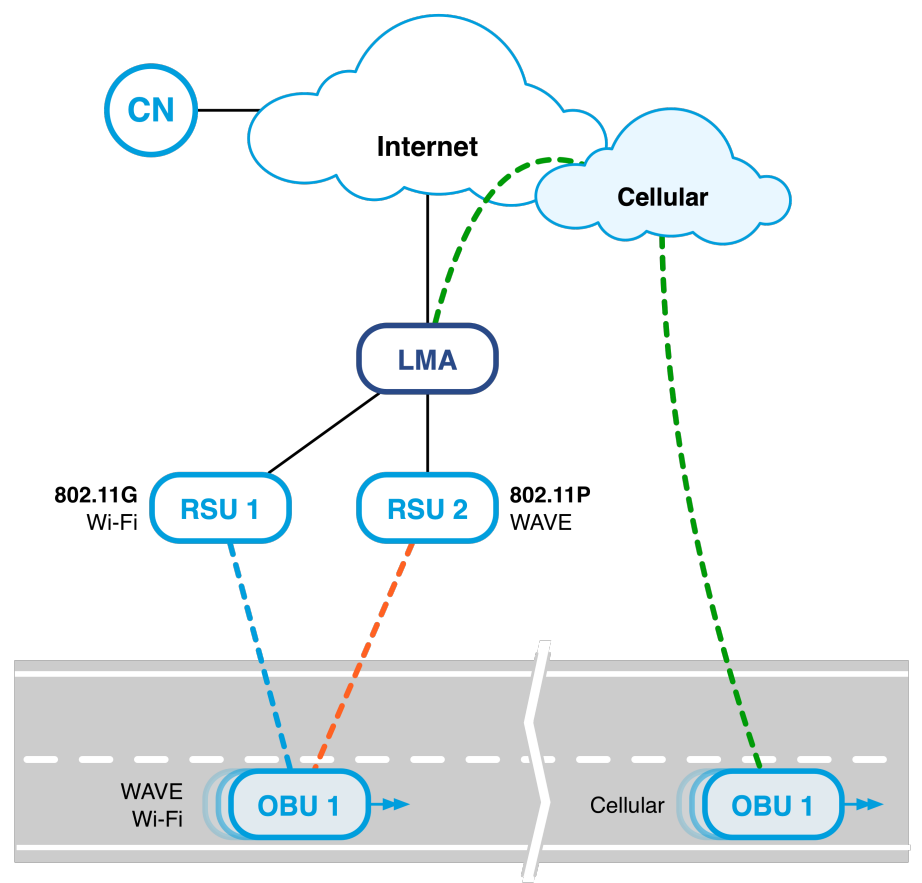

Figure 4.5: Proposed VANET architecture with the integration of cellular network. 


\subsubsection{Challenges}

Using a commercial cellular provider, or in other words, an external network to the VANET mobility solution, the mechanisms implemented for the use of WAVE and Wi-Fi cannot be replicated. For the VANET to maintain transparency and independence, some of the challenges that need to be taken into account are:

- The fact that commercial cellular networks are IPv4 networks and the VANET is based in IPv6;

- The OBU using the cellular interface will be behind a NAT box deployed by the commercial cellular provider, that may use different IP addresses for the same client over time;

- The mobility platform needs to have an optimized handover process in order to provide the most seamless experience possible when transitioning between the RSU connections (WAVE or Wi-Fi) and cellular links;

- The LMA needs to have a way of knowing if an OBU cellular connection is active, in order to allow, not only upstream communications, but also downstream communications via cellular.

\subsubsection{Proposed Approach}

The approach will consist in taking advantage of the LMA connection to the internet. Being this a public IP address, it can be used as an end-point for a communication originating at the OBU that will also have a connection to the Internet via a cellular interface. For this, a new thread, a cellular process, will be added to the OBU's connection manager alongside the existing threads (Wi-Fi thread, WAVE thread and RA thread). At the LMA, it will be added to the mobility protocol a cellular thread. This will provide a cellular process in each entity that will be responsible for processing packets to be sent or received via the cellular network.

The cellular process will allow for UDP encapsulation, at the OBU and at the LMA, and this will be done in order to maintain the original packet IP header when traversing the external networks and their NAT mechanisms (such as the NAT box deployed by the commercial cellular provider). Therefore, it will allow the LMA's cellular process to use the original IP address of the packet received in this process to know to which OBU the user is connected. Maintaining the original IP packet intact will also allow the LMA to be able to redirect the packets in downlink to the respective OBU via the vehicular network, if a connection to the OBU via a RSU becomes available. If no route is available via the vehicular network, the cellular process will make use of the IP address of the packet to send, to obtain the OBU's ID. This process maintains a linked list with the relevant information (such as the cellular IP address) related to every OBU using cellular capabilities to be able to send the packets via the cellular network.

The OBU's cellular process will take advantage of the disconnect process and associated connection status in order to trigger the use of the cellular link. Therefore, when no Wi-Fi or WAVE connection is being used by the OBU, the cellular process will take place. 
The routing process will also be optimized to allow the communication between the LMA public IP address (of the network interface connected to the Internet) and the OBU cellular interface (connected to the Internet via the commercial cellular network), and the interworking with the VANET routes when available.

A heartbeat mechanism is also proposed as part of the solution. It represents a periodic signal, in this case generated by software, in order to indicate the normal operation of the OBU to the LMA. This mechanism arises from the need to keep the LMA informed of the operational OBU when this is not directly connected to the VANET via a WAVE RSU or a Wi-Fi RSU, allowing the preservation of updated information related to the IP/Port given for this communication path by the commercial cellular NAT box. Therefore, the LMA will have the ability to know if a connection established by the OBU is still relevant or not. A connection may become irrelevant if the OBU is no longer in use (powered off), or if the information related to the IP address given at the cellular network is outdated, taking into account the cellular networks NAT UDP mapping timer.

In cellular commercial networks, the NAT table removes an entry if that given IP/Port does not have a communication flow for a specific period of time, allowing the reallocation of this resource. When developing the solution proposed in this dissertation, this challenge will have to be taken into account, since this timeout would imply the loss of the ability for the LMA to send traffic to the OBU (downstream communication).

A Delete Route (DR) mechanism is also proposed to inform the LMA cellular process that a communication session via cellular has started, and therefore, triggering the configuration of the respective routes to also allow downstream cellular communications. This process is done in order to avoid loss of packets due to the wait for the normal disconnect mechanism. The loss of packets happens when the OBU starts sending packets via cellular, immediately after losing the WAVE or Wi-Fi connection, but the LMA is still waiting for the disconnect to be received from the RSU.

Illustrated in Figure 4.6 is an example of the messages involved in a cellular communication between the OBU and LMA, as proposed by this approach. And in summary, the structure and purpose of the KA and DR messages are as follows:

- the Keep Alive (KA) message is a simple UDP packet sent by the OBU's cellular process, where the data field will be a specific string to be interpreted by the LMA's cellular process, with the OBU's ID included; it is used to keep the LMA informed of the existence of the respective OBU active with cellular capabilities;

- the Delete Route (DR) message is a simple UDP packet sent by the OBU's cellular process, where the data field will be a defined string to be interpreted by the LMA's cellular process; it is used to trigger at the LMA the configuration of the routes for downstream cellular communications. 


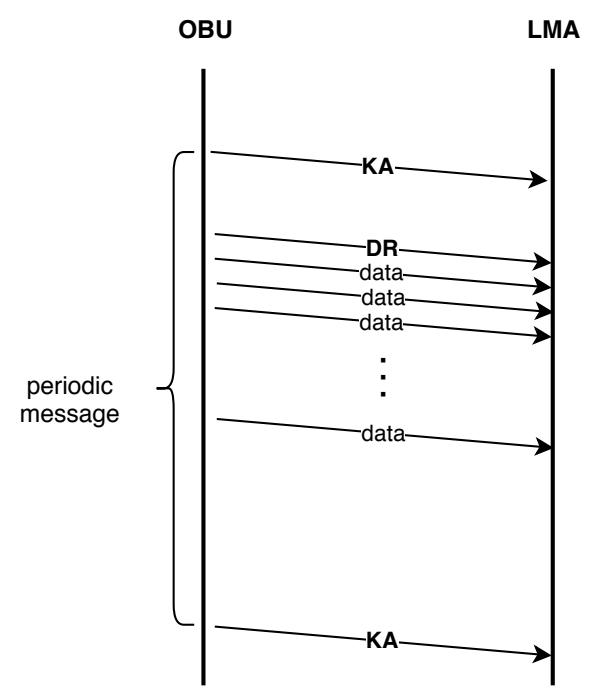

Figure 4.6: Example of VANET messages in a cellular communication between an OBU and the LMA.

\subsubsection{Overview of a Communication Scenario}

A simple overview of an example for the end-user communications can be seen in Figure 4.7. When a vehicle is within range of a WAVE RSU ("WAVE RSU 1" or "WAVE RSU 2" as presented in Figure 4.7), it will establish a connection to the infrastructure using the WAVE interface. When the connectivity via WAVE is lost, and the only available network is cellular, the network traffic will be redirected through the UDP tunnel using routes via this cellular network. When a connection is regained via a WAVE RSU, the traffic will again be redirected through the IPv4 in IPv6 tunnel.

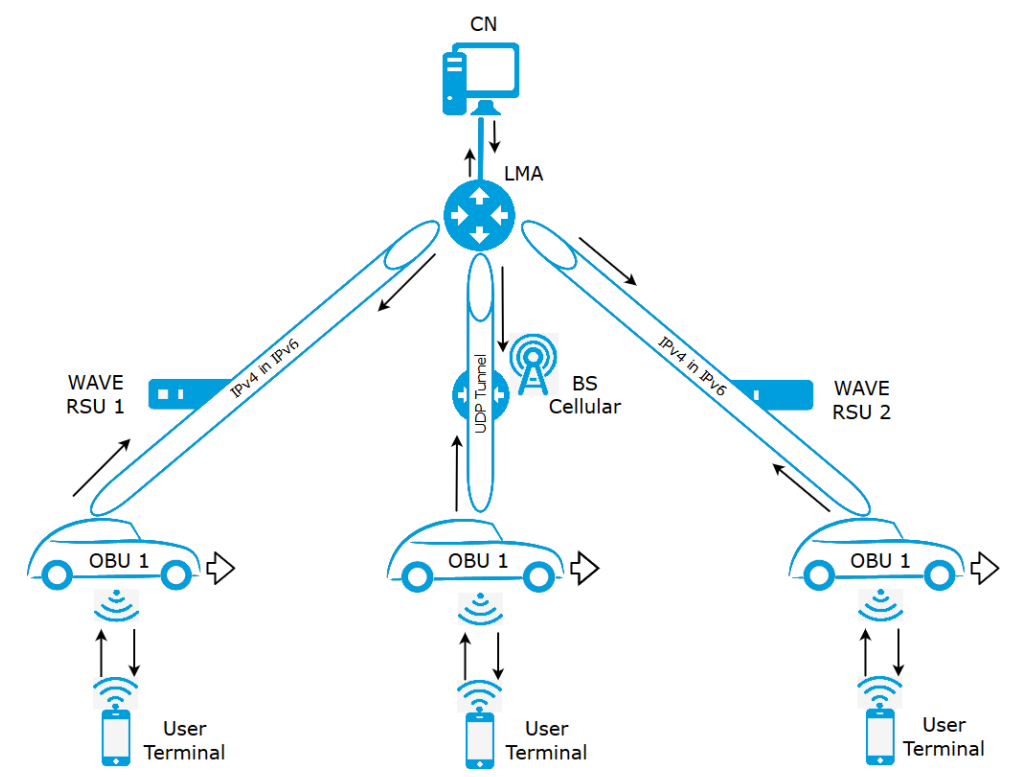

Figure 4.7: Example of end-user communications via respective tunnels. 


\subsection{Final Considerations}

This chapter presented the current architecture of the VANET, as well as the mobility solution used as base work for this dissertation, the N-PMIPv6 and the connection manager used in the OBUs. It provided a look into the N-PMIPv6 mobility protocol and respective entity operations, the LMA and the MAG/mMAG, and also explained how Internet IPv4 connection support is provided to the users.

It is presented an overview of the proposed VANET architecture, the challenges to overcome and a more in depth discussion of the proposed approach. This solution aims to improve the availability of the VANET, while maintaining its independence and transparency, by making use of commercial cellular networks as an alternative V2I communication link.

In the next chapter, it will be presented the implementation of the proposed architecture. 


\section{Chapter 5}

\section{Mobility with Cellular Communications: Implementation}

This chapter describes the implementation of the architecture presented in the previous chapter. The chapter is structured as follows:

- Section 5.1 presents the improvements made to the base project that will also work as a preparation for the implementation of the cellular solution;

- Section 5.2 presents the operation at the OBU of the connection manager with the cellular solution implementation;

- Section 5.3 presents the operation of the N-PMIPv6 protocol at the LMA entity with the cellular solution implementation;

- Section 5.4 presents the heartbeat mechanism developed in order to overcome some challenges introduced by the nature of commercial cellular networks;

- finally, Section 5.5 presents the main ideas discussed in this chapter, providing a summary of the solution implemented.

\subsection{N-PMIPv6 and Connection Manager Improvements}

During this work, several improvements were added to the VANET's connection manager and mobility protocol, focused mainly in the verification of available wireless connections in each OBU aiming to include all the possible use cases to be tackled by the proposed architecture. This way, the cellular interface can be used depending on the number and quality of WAVE and Wi-Fi links currently available.

A dynamic configuration of the $\mathrm{IPv} 4$ tunnels and respective routes between the LMA and OBU was implemented to allow real-time configuration, by the system, of the necessary routing procedures based on the technologies in use at the OBU to provide Internet access to its users. It was taken into account the previous implementation of the user Internet $\mathrm{IPv} 4$ support through OBUs, and a new mechanism was implemented in the OBU's connection manager and the LMA mobility protocol. A new standard for the IP address assignment process between the OBU (as a Wi-Fi AP) and its end-users was defined, in order to facilitate the process of dynamic routes, and to allow a better organization of the network. It uses the 
board ID (a unique three digit number that identifies the OBU, and, for this purpose, are used the two first right digits) as follows:

$$
\text { 10.0.Board } I D(\bmod 100) .0 / 24 \text {. }
$$

The LMA mobility protocol will make use of the board ID obtained from the IP header of the packet sent by the OBU, and create the respective IPv4 tunnels and routes for the OBU (and, therefore, its end-users). This will also be useful for the LMA cellular process to use the IP header to identify the OBU communicating via a cellular network. The OBU will also create the respective $\mathrm{IPv} 4$ tunnel and route based on which technology it is connecting to (WAVE or Wi-Fi), and delete them in case of a disconnect. These changes were made, not only for the improvement of the mobility platform, but also in preparation for the cellular solution implementation.

\subsection{Connection Manager Cellular Operation}

First, the OBU needs to have the hardware for cellular capabilities; we used the module "Novatel EU850D - 3G/HSDPA Mini PCI Express Card". The cellular provider does not need to be specified, since the proposed solution works no matter the network operator in use. The important aspect is for the cellular network to be seen as an IP network with connection to the Internet, in order to provide a path to the LMA public IP address, which is achieved by configuring the network interface with the respective options according to the chosen provider, allowing for the OBU to use the new configured cellular interface to access the Internet.

In order to make use of an UDP tunnel via the cellular connection as a V2I link between the OBU and the LMA, we will first be using a tun/tap device, in this case tun since it is a softwareonly interface, to simulate a network layer device operating at layer 3 carrying IP packets utilized at the user-space, so that the packets can be processed, therefore allowing the UDP encapsulation process. Illustrated in Figure 5.1 is an overview of the cellular communication between the VANET entities to provide the reception and transmission of packets, passing through the cellular network and Internet, making use of the tun device and the respective interface connected to the internet, going through an UDP tunnel.

A default route with a higher metric than the routes for WAVE and Wi-Fi is applied to this tun device. This way, in the absence of WAVE and Wi-Fi connections, the network traffic can go through the tun device.

Another route to the public IP address of the LMA is also inserted in the OBU via the cellular interface, redirecting through this interface all the traffic with that destination (the public IP address of the LMA). This will make all network traffic from the tun device to go through the cellular network. As mentioned, the tun device will serve for the cellular process to encapsulate the packets, using UDP, and redirect the traffic to the LMA endpoint.

A new thread responsible for the cellular solution process was implemented in the connection manager; Figure 5.2 illustrates its execution flow, that will now be explained. With the start of the connection manager, the thread of the cellular operation also starts, opening the tun, and creating the UDP socket and performing the binding. Then, two specific messages will be created, the KA and the Delete Route (DR). 


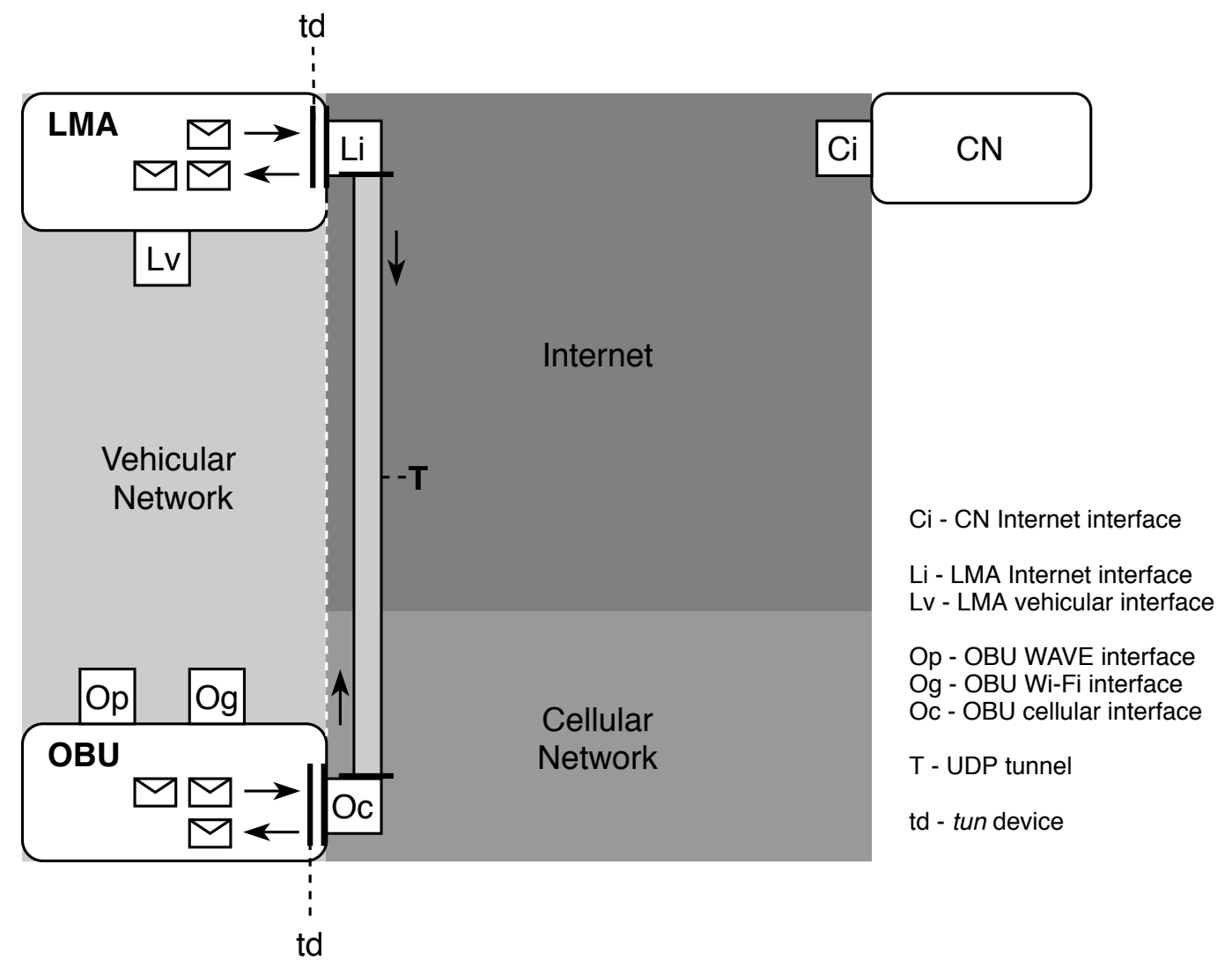

Figure 5.1: Overview of cellular communication between the OBU and the LMA.

After the creation of these messages, the first KA message, with the respective board ID, informs the LMA that a new OBU has started operations and has cellular capabilities. The connection manager proceeds as expected, periodically scanning for WAVE and Wi-Fi connections and executing the normal process of connecting and using these access networks when they are available.

When no WAVE or Wi-Fi connection is found, a cellular session takes place. It is verified if the DR message was already sent for this cellular session and, if it has not been sent, as previously mentioned, it is needed to inform the LMA that the OBU will send network traffic via the cellular connection and the other routes must be removed. Additionally, WAVE and/or Wi-Fi default routes at the OBU will be deleted, so that the traffic correctly goes through the tun device.

When a WAVE and/or Wi-Fi connection is found again, and after a success connection establishment where the vehicular routes are again configured, the cellular process is put on hold, until needed again, resetting the status of the DR mechanism, allowing the normal procedure of sending the DR message through this communication channel for future cellular uses. 


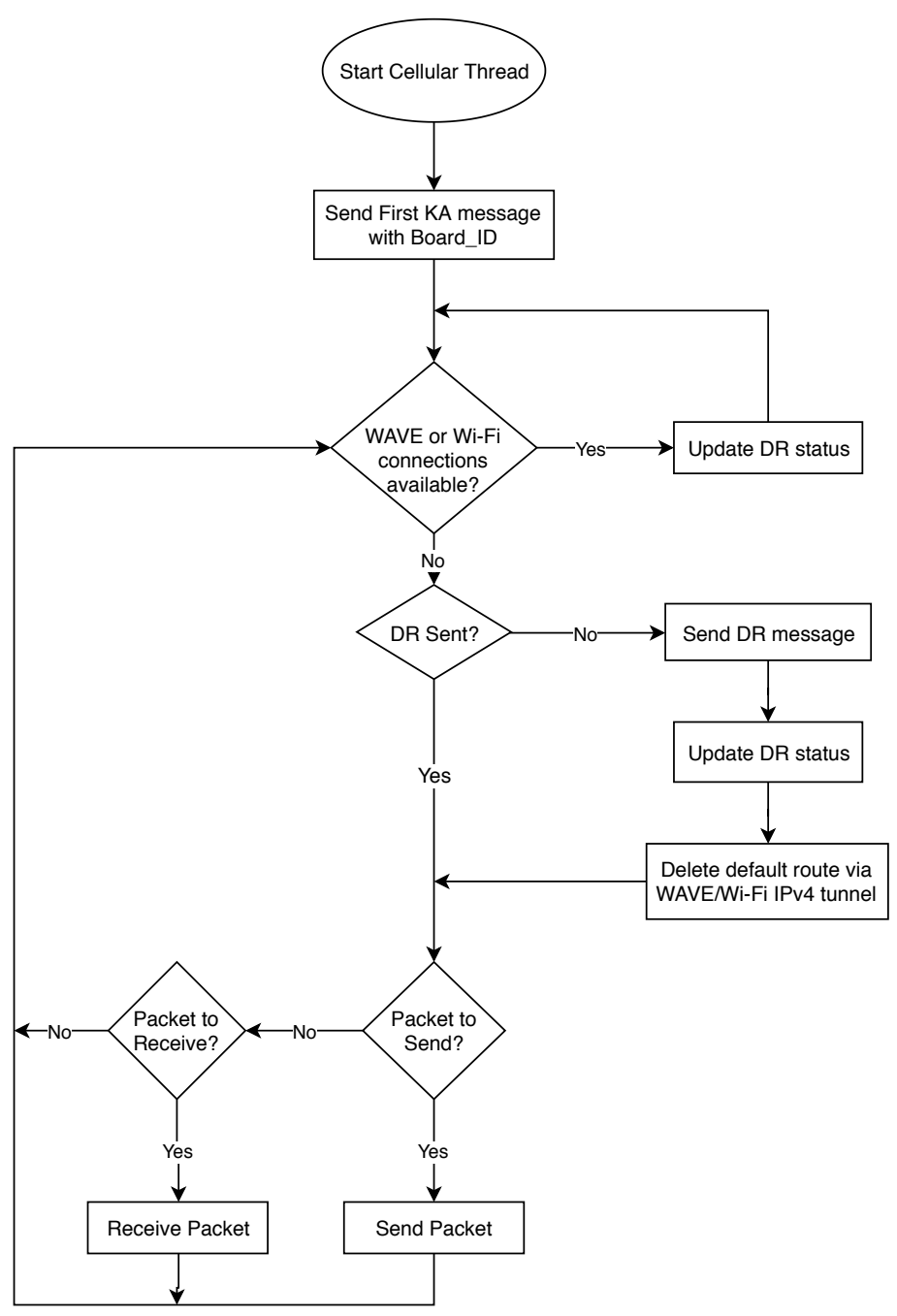

Figure 5.2: Connection manager cellular operation work flow.

\subsubsection{Disconnect Mechanism}

It was also implemented in the connection manager a new disconnect mechanism interworking with the existing one, since the base work only contemplated the use of WAVE and Wi-Fi. For example, when connected to a WAVE RSU, the OBU would only disconnect from this technology when the disconnect threshold value was reached, and since there was no alternative, this value was defined as the minimum possible for a connection to be maintained over WAVE. On the previous implementation of the mobility solution, the disconnect threshold value $(D T H)$ was defined to a RSSI value of 10 , and the connect threshold value to a RSSI value of 30. But, with this new cellular solution, there is no need for a downtime when no WAVE connection is available. Nevertheless, it is important to maintain the independence of the current VANET architecture and implement this disconnect case to occur only if an OBU has cellular capabilities, and also having the disconnect occur before the WAVE connection starts to underperform while keeping in mind that the use of commercial cellular networks have costs associated. Therefore, a new disconnect case was implemented that occurs if an 
OBU is equipped with cellular and is triggered when: $R S S I_{\text {current }}<D T H+10$, if the current mean value RSSI of the WAVE connection becomes inferior to the disconnect threshold value with an increment of 10 units, a disconnect occurs. The incremented value was chosen based on tests performed. This means that the disconnection process will occur even with the OBU still in range of a WAVE signal but the signal is weak. This avoids any abrupt disconnect from the WAVE RSU in weak signal conditions in order to minimize the loss of packets due to the use of such weak links. A reconnect to a RSU will occur when the RSSI becomes equal or greater than the defined connect threshold value, corresponding to a value that ensures a steady WAVE connection and, also, does not overlap with the disconnect approach.

\subsection{LMA Cellular Operation}

The LMA, as the central management entity of the VANET, is directly connected to the Internet. Since the LMA can learn the public IP address of an OBU using the cellular solution, it will be able to communicate to this address.

As also mentioned in Section 5.2, a tun device is used by the N-PMIPv6 protocol to process the packets at the user-space. At the start of the protocol, the cellular process also starts, opening the tun, the UDP socket is created and the binding is completed, so that packets can be received and sent during the execution of this process. Illustrated in Figure 5.3 is the execution flow for the cellular operation at the LMA that will next be explained.

If an uplink packet is received in the tun device, first it is verified if the message corresponds to a $\mathrm{KA}$, and, if that is the case, it is checked if it exists an entry for the respective board that is the message original sender. If an entry for this board does not exist, then it is created the entry in the linked list with the following information: the board ID, the IP address and port, and, also, the timestamp related to the reception of the KA. If such entry exists, it is updated the info (IP address, port and timestamp of the reception of the last KA related to the respective board).

If the uplink message received in the tun device is a DR message, it will be called the procedure to delete the WAVE and/or Wi-Fi IPv4 tunnel routes for the respective OBU, and it will be added the route via the tun device. Otherwise, if it is neither a KA nor a DR, it is a normal packet being forwarded from the OBU. The LMA will route the packet, possibly to its CN, as it would do for other access technologies of the VANET.

If the LMA receives a packet for downlink and there is no vehicular route (through WAVE or $\mathrm{Wi}-\mathrm{Fi}$ ), it is redirected through the tun device, where the cellular process will verify if an entry for the destination OBU exists in the linked list of known OBUs with a communication path via cellular networks. If there is an entry, a verification of this entry is made to determine if it is outdated by checking the timestamp of the entry that corresponds to the time of reception of the last KA message. And if the entry is indeed outdated, it will be deleted. Otherwise, it will be used to obtain the necessary information related to the OBU public IP address in the cellular network, in order to send the packet to the respective OBU, using that cellular network.

It is important to underline that the DR mechanism sends a packet from the cellular process through the UDP tunnel containing only a specific string message to be interpreted by the LMA's cellular process, in order to identify the correct packet as a DR message and no other packet. This triggers the change of routes at the LMA as previously mentioned, and comes as a solution for the delay that the VANET has when a connection is lost. A 


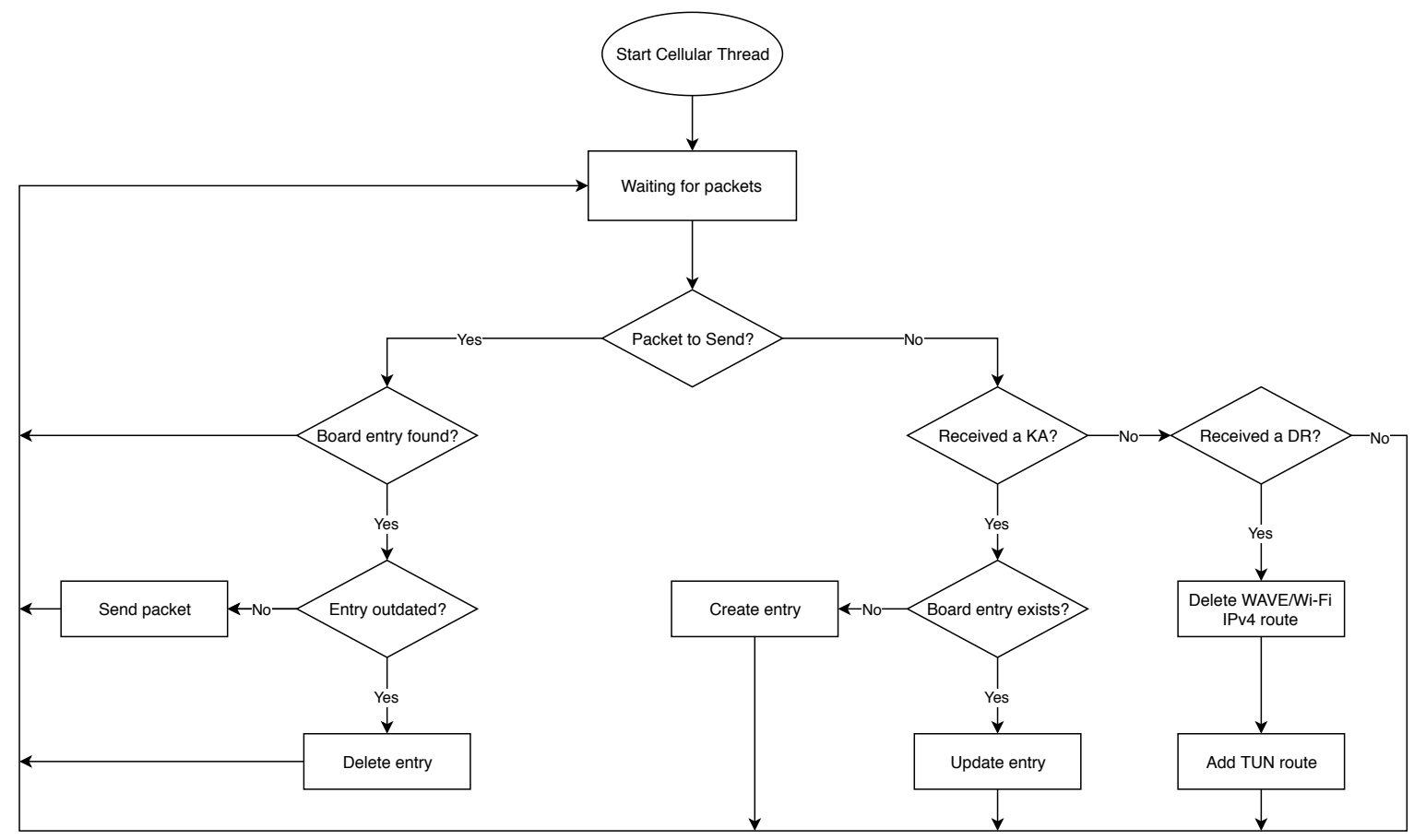

Figure 5.3: LMA cellular operation flow diagram.

period of waiting for a new vehicular connection and attempt at retransmissions previously defined as 45 seconds was changed in this implementation, nevertheless a small delay persists and due to the LMA maintaining the route to the WAVE or Wi-Fi tunnel until it receives the disconnect from the RSU (even when it is already receiving traffic in the tun device from the OBU cellular interface), it requires the implementation of the DR mechanism. Therefore, with this mechanism, the LMA is able to configure the cellular route at the moment the OBU makes use of this connection, being able to communicate in downstream with the correct link in use by the OBU.

An added aspect that needs to be referred is that the cellular link continues to be used for a brief specific time after a WAVE or Wi-Fi link is again found. The timing for the procedure of a new connection to be established at the OBU, due to being performed by protocol operations and taking into consideration the limitations of the hardware used, takes approximately two seconds after a WAVE or Wi-Fi connection is found and consequently for that link to be used for routing. The message PBU sent to the LMA will trigger the configuration of the vehicular interface (route) to be used for the communications before the OBU is indeed ready to use that medium, and therefore the LMA delays the addition of the routes via the IPv4 tunnels for the WAVE and Wi-Fi connection in order to maintain the use of the cellular link during this setup period, avoiding significantly greater packet losses in the handover process. 


\subsection{Heartbeat Mechanism}

As proposed, it was implemented a heartbeat mechanism consisting of a thread in the connection manager (executed by the OBU) that sends a message called Keep Alive (KA), containing the respective board ID, to be interpreted by the LMA's cellular process, in order to identify the correct packet as a KA message and no other packet, and to obtain the OBU's ID from which this message originated (this packet will not use the standard IPv4 address as is used in the normal user communication, because it originates in the cellular process and is sent via the UDP tunnel). This message is sent periodically, and the time value for the periodic sending of the KA message was chosen empirically, but took into consideration NAT mapping timer present in commercial cellular NAT boxes, and a relevant time window for the LMA to maintain a certain assurance of the availability of the connections.

The KA message is processed at the LMA. When the LMA receives a KA message in the cellular process, it verifies if it is indeed a correctly defined KA message, and taking the board ID from this received message, it will check for an entry in the linked list that this entity maintains in order to access the information about the mobile nodes making use of a cellular network. If no entry is found, the LMA will create a new entry with the board ID, the interface (IP address and port) information and a timestamp of the reception of this KA message; if an entry is found, the LMA will update the information for this OBU entry, either updating the IP address and/or port or only updating the timestamp of the reception of the KA message, as referred in Section 5.3.

When the LMA needs to send a packet through cellular technology to an OBU, the LMA cellular process will check the linked list for an entry that corresponds to the respective OBU ID, in order to obtain the necessary information to send the packet via the cellular network. If an entry is found, the cellular process verifies if it is outdated by comparing the timestamp and a defined value of the allowed maximum valid time window for an entry information to be considered accurate. In the case of the information being considered outdated, the entry for the specific node will be deleted and the packet will be discarded. Otherwise, it will use this information to send the packet. If the entry is not found, it means that there is no path, for the time being, to communicate with the respective OBU and the packet will be discarded.

\subsection{Final Considerations}

This section presented and discussed the implementation of the proposed cellular approach for the VANET. The main aspects that this work allowed to develop for the VANET platform were as follows:

- New network access solution, using commercial cellular networks, as a backup for V2I communications between OBUs and LMA;

- Keep-Alive mechanism to allow downstream communications, between the LMAs and OBU, via commercial cellular networks;

- New disconnect mechanism with the cellular integration, to avoid packet loss due to weak WAVE signal connection;

- Multiple OBU's cellular connections supported at the LMA; 
- Handover process improvement for the integration of the cellular solution;

- Transparency to the end-users connected to the OBUs.

In summary, the mobility protocol now makes use of WAVE, Wi-Fi and cellular technologies to maintain V2I communications, avoiding downtime due to lack of connectivity from RSUs that the OBUs could experience. In the next chapter it will be presented and discussed several tests conducted in order to corroborate the work developed in this dissertation. 


\section{Chapter 6}

\section{Evaluation}

This chapter presents the results for the experiments focusing on the correct integration of the proposed solution, and on the evaluation of its performance, and is divided in the following topics:

- Section 6.1 presents the equipment (hardware and software) used in the tests;

- Section 6.2 presents and discusses the evaluation of the new VANET solution, both in laboratory and real-world tests;

- finally, Section 6.3 summarizes the chapter's contributions.

\subsection{Hardware and Software}

The proposed solution was evaluated using real VANET equipment currently deployed in several city-scale VANETs, namely the communication modules, the Road-Side Units (RSUs) and the On-Board Units (OBUs). Below is a complete list of all the equipment used in the evaluation process:

- Acting as Correspondent Node $(\mathrm{CN})$, the end-point for the tests using the Distributed Internet Traffic Generator (D-ITG), was used a computer with the characteristics presented in Table 6.1;

- The same equipment used to host the CN was also used to host the LMA. However, in this case, this entity was virtualized running the VirtualBox5.2.8, a program from Oracle for virtual machines, that used the guest with the characteristics presented in Table 6.2 in order to run the N-PMIPv6 mobility protocol as the LMA;

- For the RSUs it were used NetRiders (version 3), single board computers containing IEEE $802.11 \mathrm{p}$ and IEEE $802.11 \mathrm{a} / \mathrm{b} / \mathrm{g}$, that run the N-PMIPv6 mobility protocol as MAGs. These boards have the characteristics presented in Table 6.4, for the exception of not having the $3 \mathrm{G} / \mathrm{HSDPA}$ module;

- The OBUs is also a NetRider, containing IEEE 802.11p, IEEE $802.11 \mathrm{a} / \mathrm{b} / \mathrm{g}$, and, in this case, the cellular capabilities (using the module "Novatel EU850D - 3G/HSDPA Mini PCI Express Card"), with the characteristics presented in Table 6.4, that runs both the N-PMIPv6 mobility protocol as mMAGs and the connection manager; 
- A computer with the characteristics presented in Table 6.3 was used to act as client, placed inside the vehicle and connected to the OBU through cable, in order to remove the entropy associated to a wireless connection between the client and the OBU for the intended tests.

\begin{tabular}{|ll|}
\hline CPU & Intel®Core ${ }^{\mathrm{TM}} \mathrm{i} 5-3317 \mathrm{U} @ 1.70 \mathrm{GHz}$ (2 cores) \\
OS & Ubuntu 18.04.1 LTS \\
Linux Kernel & $4.15 .0-54$-generic \\
RAM & $6 \mathrm{~GB}$ \\
\hline
\end{tabular}

Table 6.1: CN and LMA Host Equipment characteristics.

\begin{tabular}{|ll|}
\hline CPU & Intel $\AA$ Core $^{\mathrm{TM}} \mathrm{i} 5-3317 \mathrm{U} @ 1.70 \mathrm{GHz}$ (2 cores) \\
OS & Ubuntu $16.04 .3 \mathrm{LTS}$ \\
Linux Kernel & 4.14 .3 \\
RAM & $1 \mathrm{~GB}$ \\
\hline
\end{tabular}

Table 6.2: LMA (Virtual Machine) characteristics.

\begin{tabular}{|ll|}
\hline CPU & Intel@Core ${ }^{\mathrm{TM}} \mathrm{i} 7-3635 \mathrm{QM} @ 2.40 \mathrm{GHz}$ (8 cores) \\
OS & Ubuntu 18.04.3 LTS \\
Linux Kernel & 4.15.0-64-generic \\
RAM & 8 GB \\
\hline
\end{tabular}

Table 6.3: Client equipment characteristics.

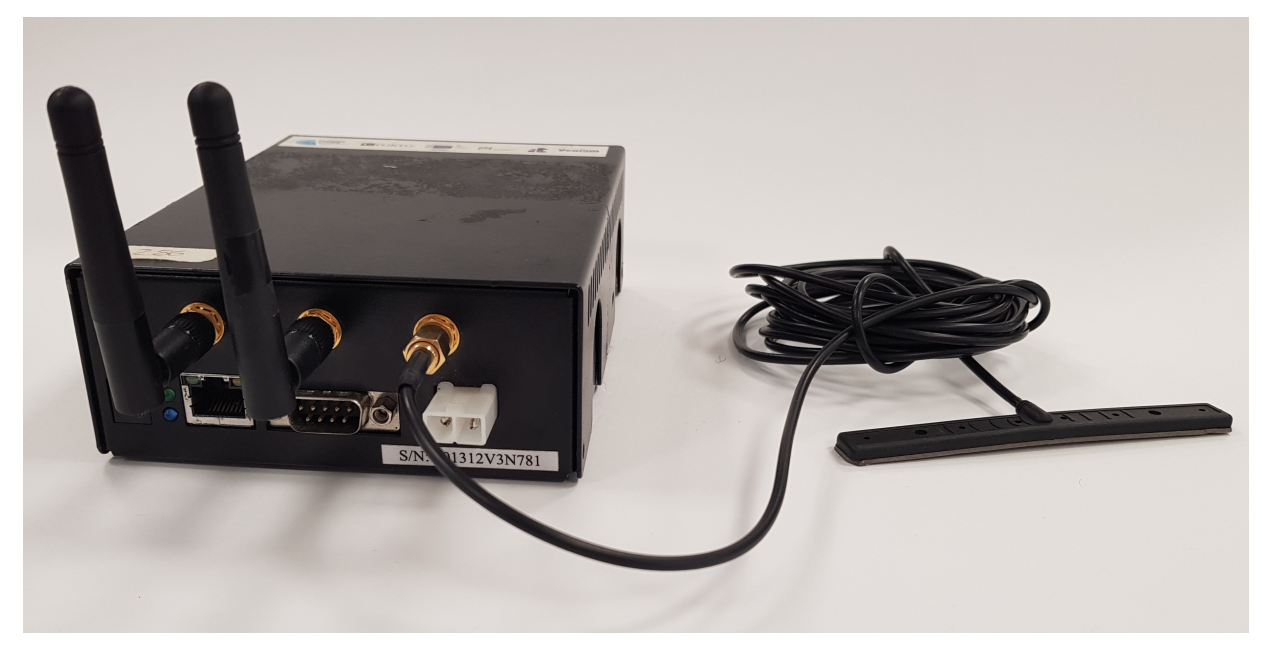

Figure 6.1: NetRider version 3. 


\begin{tabular}{|ll|}
\hline CPU & Atheros AR7161 MIPS32 680 MHz \\
OS & VeniamOS 19.2 \\
Linux Kernel & Linux 3.7.4 MIPS \\
RAM & $64 \mathrm{MB}$ \\
Disk & $128 \mathrm{MB}$ \\
Modules & IEEE $802.11 \mathrm{a} / \mathrm{b} / \mathrm{g}, 802.11 \mathrm{p}$ and 3G/HSDPA \\
Ports & Ethernet, serial (RS-232) and USB \\
\hline
\end{tabular}

Table 6.4: NetRider Specifications.

The D-ITG tool [52], a software capable of producing traffic at packet level, accurately replicating appropriate stochastic process for both Inter Departure Time and Packet Size random variables, was used to extract the evaluation results with respect to the proposed solution. It also supports both IPv4 and IPv6 traffic generation but for the intended tests in this dissertation it was only generated IPv4 traffic. The protocol used in the tests was UDP, the default for D-ITG, in order to obtain the packet loss.

\subsection{Tests}

Figure 6.2 illustrates the evaluation scenario (used for scenario 1 in laboratory and realworld conditions). The setup consisted in loss of connection situations where a mobile node connected to a WAVE RSU moving along a road and eventually losing connection to the WAVE RSU, starts using the cellular technology until a new WAVE connection is found again. This scenario was compared with two distinct scenarios, with constant connectivity to the infrastructure for a better evaluation of the VANET, testing the improvements on the N-PMIPv6 mobility protocol and connection manager. The three evaluation scenarios are described as follows:

- Scenario 1 - based on Figure 6.2, was used to evaluate the performance of both mobility solutions:

- Base Solution - corresponds to the use of the VANET without cellular, and therefore a downtime occurs when no RSU is reachable;

- Cell Solution - corresponds to the use of the VANET with the cellular approach, where the cellular connection is used when needed, triggering an handover between technologies.

- Scenario 2 - Consists in a stable WAVE connection for the entirety of the experiment time window (50 seconds). This scenario was introduced to better explain some results achieved during scenario 1 ;

- Scenario 3 - Consists in a stable cellular connection for 15 seconds. This scenario was introduced to better explain some results achieved during scenario 1. 


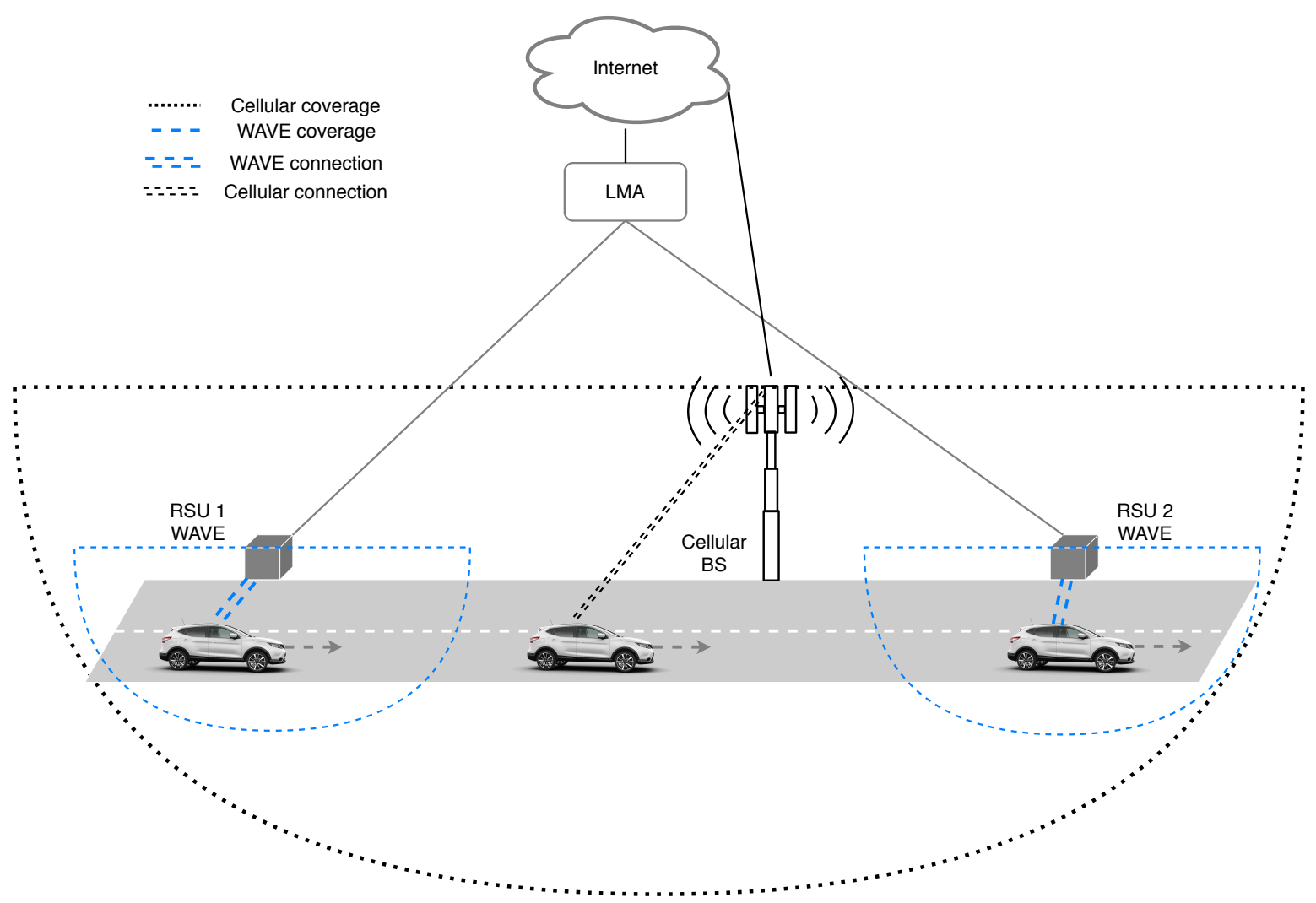

Figure 6.2: Evaluation scenario 1.

The evaluation tests focused on the three scenarios presented before, considering four distinct types of tests with different packet sizes and packet rates, as presented in Table 6.5. Each test was performed 10 times in laboratory conditions and 5 times in real-world conditions, and are presented with a $95 \%$ confidence interval. The specific parameters for each test are presented in Table 6.5.

\begin{tabular}{c|cc} 
Test & Packets/second & Bytes/packet \\
\hline \hline $\mathrm{A}$ & 25 & 500 \\
$\mathrm{~B}$ & 25 & 750 \\
\hline $\mathrm{C}$ & 100 & 500 \\
$\mathrm{D}$ & 100 & 750
\end{tabular}

Table 6.5: Parameters for each test.

It should be noted that during each test conditions, there is no manual manipulation of the VANET operations, namely routing tables and tunnel creations. The systems are set running and all functionalities evaluated in this chapter are, as expected, automatically performed by the VANET. The results, as it will be shown, are directly affected by the characteristics of each technology, environmental factors, the respective scenario and the test parameters. 


\subsubsection{Laboratory Tests}

For the laboratory tests a timeline was defined. The time window for these tests can be observed in Figure 6.3, where a loss of connection with the WAVE RSU occurs, approximately, at instant $\mathrm{t}=10$ seconds and a new connection to a WAVE RSU occurs, approximately, at instant $\mathrm{t}=25$ seconds, for scenario 1 .

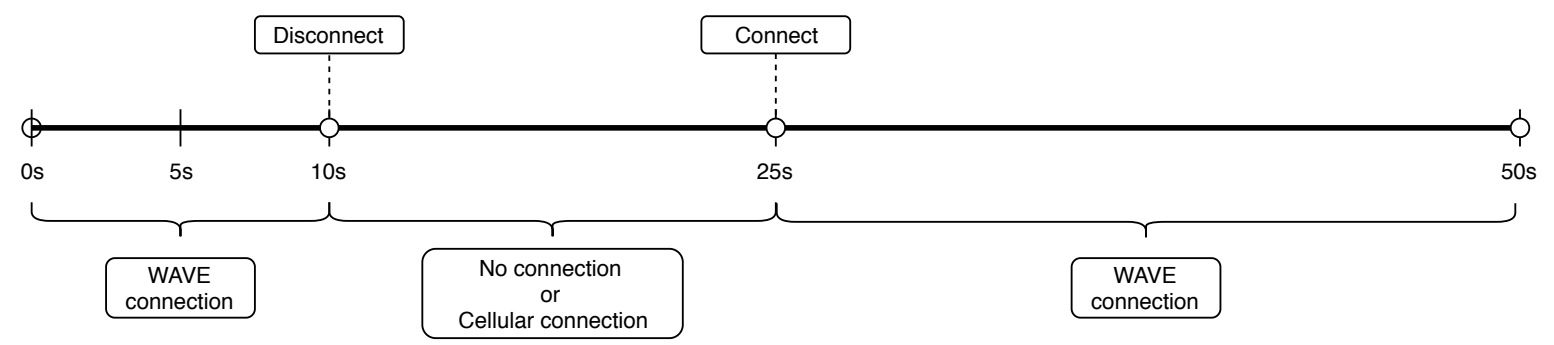

Figure 6.3: Scenario timeline.

Let us start by analysing the Base Solution over scenario 1. In this case, the OBU without cellular capabilities loses connectivity with the first RSU WAVE at instant $\mathrm{t}=10$ seconds and connects to a new WAVE connection at instant $\mathrm{t}=25$ seconds, being without connection in the meantime. The performance results with respect to packet loss, bitrate and packet rate are illustrated in Table 6.6.

\begin{tabular}{c|ccc} 
Test & Packet loss $(\%)$ & Bitrate $(\mathrm{kbit} / \mathrm{s})$ & Packet rate $(\mathrm{pkt} / \mathrm{s})$ \\
\hline \hline $\mathrm{A}$ & $\mathbf{3 7 . 4 6} \pm 0.65$ & $62.79 \pm 0.65$ & $15.70 \pm 0.16$ \\
$\mathrm{~B}$ & $\mathbf{3 7 . 3 2} \pm 0.56$ & $93.99 \pm 0.84$ & $15.66 \pm 0.14$ \\
\hline $\mathrm{C}$ & $\mathbf{3 7 . 7 6} \pm 0.68$ & $248.10 \pm 2.64$ & $62.02 \pm 0.66$ \\
$\mathrm{D}$ & $\mathbf{3 7 . 5 0} \pm 0.76$ & $372.68 \pm 4.36$ & $62.11 \pm 0.73$
\end{tabular}

Table 6.6: Results for the Base Solution in laboratory conditions.

These values refer to a time window of 50 seconds, and the OBU was out of range and consequently without connectivity during approximately 15 seconds. With the results obtained in Table 6.6 it is possible to verify that the packet loss values go from $37.32 \%$ (for test B) up to $37.76 \%$ (for test $\mathrm{C}$ ), the minimum and maximum values, respectively, of the four tests, therefore being similar. Moreover, the bitrate for test A and test B are respectively 62.79 $\mathrm{kbit} / \mathrm{s}$ and $93.99 \mathrm{kbit} / \mathrm{s}$, the difference is justified for the input parameter of 500 bytes per packet for test $\mathrm{A}$ and of 750 bytes per packet for test B; the bitrate for test C and test D are respectively $248.10 \mathrm{kbit} / \mathrm{s}$ and $372.68 \mathrm{kbit} / \mathrm{s}$ and have the same justification provided for test $\mathrm{A}$ and $\mathrm{B}$, but the increase compared to these former tests is due to the input parameter packet rate being 100 for these tests $\mathrm{C}$ and $\mathrm{D}$ instead of 25 for tests $\mathrm{A}$ and $\mathrm{B}$, and this will also justify the similarities of values obtained for the packet rate in test $\mathrm{A}$ and $\mathrm{B}$ compared to the obtained results for test $\mathrm{C}$ and $\mathrm{D}$. These values obtained do not achieve the full network performance due to being an average obtained for the time window and a loss of connectivity is verified between the OBU and the RSUs, downtime that in this time window will correspond to approximately $37 \%$ of packet losses for the VANET. 
The obtained values, in the first scenario using the Base Solution, will be taken as the base for comparison with the results that refer to the developed work in this dissertation, that aims to avoid such packet loss when in a downtime scenario as the one depicted in this case.

The Cell Solution, in scenario 1, is the one that presents the results for the tests that evaluate the improvements proposed in this work. The scenario is the same as before, but now the OBU is able to use the cellular technology in the absence of RSUs or when the WAVE link is below the threshold. The performance results with respect to packet loss, delay, jitter, bitrate and packet rate are illustrated in Table 6.7.

\begin{tabular}{c|ccccc} 
Test & Packet loss $(\%)$ & Delay $(\mathrm{ms})$ & Jitter $(\mathrm{ms})$ & Bitrate $(\mathrm{kbit} / \mathrm{s})$ & Packet rate $(\mathrm{pkt} / \mathrm{s})$ \\
\hline \hline A & $\mathbf{3 . 2 5} \pm 0.75$ & $27.89 \pm 7.26$ & $0.950 \pm 0.139$ & $96.29 \pm 0.74$ & $24.07 \pm 0.19$ \\
B & $\mathbf{3 . 9 9} \pm 0.27$ & $18.33 \pm 4.06$ & $0.924 \pm 0.111$ & $143.54 \pm 0.40$ & $23.95 \pm 0.07$ \\
\hline C & $\mathbf{2 0 . 5 0} \pm 0.37$ & $93.58 \pm 3.69$ & $0.551 \pm 0.015$ & $317.32 \pm 1.50$ & $79.33 \pm 0.37$ \\
D & $\mathbf{2 1 . 5 3} \pm 0.68$ & $90.38 \pm 7.91$ & $0.584 \pm 0.030$ & $468.53 \pm 3.95$ & $78.09 \pm 0.66$
\end{tabular}

Table 6.7: Results for the Cell solution in laboratory conditions.

Let us start by analysing the results obtained in relation to the different tests evaluated with this solution, noticing that for tests $\mathrm{A}$ and $\mathrm{B}$ the packet loss is very low compared to tests $\mathrm{C}$ and $\mathrm{D}$. These results show that an increase in the packet rate affects significantly the performance of the VANET using the cellular solution, and further insights will be provided related to the performance of the cellular connection with high packet rate.

Proceeding by comparing the results obtained with the cellular solution to the ones obtained with the non-cellular solution. As for test A, characterized by an UDP traffic of 25 packets per second with a packet size of 500 bytes, we can see a packet loss of $3.25 \%$ where previously, without the implementation of the cellular solution, the packet loss was of $37.46 \%$, meaning that there is a reduction of $34.21 \%$ in the number of packets dropped when compared to the non-cellular mobility solution. For test B, 25 packets per second with a packet size of 750 bytes, the packet loss obtained was $3.99 \%$, where without the cellular solution the value corresponded to $37.32 \%$, meaning a difference of minus $33.33 \%$. For test C, characterized by an UDP traffic of 100 packets per second with a packet size of 500 bytes, it is obtained a packet loss of $20.50 \%$ and the value obtained in the test runs without the cellular solution was $37.76 \%$, meaning a difference of minus $17.26 \%$. For test D, 100 packets per second with a packet size of 750 bytes, the packet loss corresponds to $21.53 \%$ where without the solution was $37.50 \%$, meaning a difference of minus $15.97 \%$. These results show that the VANET with the cellular solution is capable of using this new technology as an alternative for communications when the other traditional VANET technologies are unavailable, the handover is fast introducing only a small packet loss in the process, and this solution does not require any contribution by the end-user or the cellular network provider. It is also possible to observe that a higher number of packets influences negatively the performance of the VANET. Additionally, increase of packet size also shows to have an impact in the performance of the VANET but not significantly as the packet rate.

The values presented in Table 6.7 do not provide a basis to take final conclusions about the real gain of the solution implemented due to having the inherent losses of the commercial cellular network. Unless stated otherwise, the commercial network provider used for the tests 
was NOS (also known as ZON OPTIMUS). So, to better examine and justify the results obtained, and consequently get the real packet losses that this solution (without the commercial network losses) brings to the respective VANET, we need to analyse the packet losses inherent to the WAVE connection and to the cellular network being used for the tests. As previously mentioned, it is also useful to provide values for the performance of the VANET under two scenarios without disconnectivity to better understand the losses introduced by each wireless communication technology. Table 6.8 presents the performance results under scenario 2, where it is maintained a WAVE connection for the entirety of the time window of 50 seconds, and Table 6.9 presents the results for scenario 3, where it is maintained a connection to the VANET via the cellular technology for the entirety of the time window in this case being 15 seconds.

\begin{tabular}{c|ccccc} 
Test & Packet loss $(\%)$ & Delay $(\mathrm{ms})$ & Jitter $(\mathrm{ms})$ & Bitrate $(\mathrm{kbit} / \mathrm{s})$ & Packet rate $(\mathrm{pkt} / \mathrm{s})$ \\
\hline \hline $\mathrm{A}$ & $\mathbf{0 . 0 0} \pm 0.00$ & $0.37 \pm 0.01$ & $0.008 \pm 0.003$ & $99.86 \pm 0.01$ & $24.97 \pm 0.00$ \\
$\mathrm{~B}$ & $\mathbf{0 . 0 0} \pm 0.00$ & $0.41 \pm 0.00$ & $0.008 \pm 0.001$ & $149.79 \pm 0.01$ & $24.96 \pm 0.00$ \\
\hline $\mathrm{C}$ & $\mathbf{0 . 1 0} \pm 0.20$ & $0.08 \pm 0.00$ & $0.008 \pm 0.004$ & $395.96 \pm 0.83$ & $98.99 \pm 0.21$ \\
$\mathrm{D}$ & $\mathbf{0 . 0 0} \pm 0.00$ & $0.10 \pm 0.01$ & $0.005 \pm 0.001$ & $594.69 \pm 0.17$ & $99.11 \pm 0.03$
\end{tabular}

Table 6.8: Results for 50 seconds of WAVE connection, scenario 2, in laboratory conditions.

\begin{tabular}{c|ccccc} 
Test & Packet loss $(\%)$ & Delay $(\mathrm{ms})$ & Jitter $(\mathrm{ms})$ & Bitrate $(\mathrm{kbit} / \mathrm{s})$ & Packet rate $(\mathrm{pkt} / \mathrm{s})$ \\
\hline \hline A & $\mathbf{1 . 8 7} \pm 1.50$ & $25.89 \pm 5.10$ & $1.795 \pm 0.253$ & $100.25 \pm 0.43$ & $25.06 \pm 0.11$ \\
B & $\mathbf{2 . 1 9} \pm 1.46$ & $21.27 \pm 4.36$ & $1.755 \pm 0.248$ & $147.55 \pm 2.24$ & $24.59 \pm 0.37$ \\
\hline C & $\mathbf{7 . 7 4} \pm 0.34$ & $838.52 \pm 8.08$ & $1.459 \pm 0.044$ & $185.23 \pm 0.54$ & $46.31 \pm 0.13$ \\
D & $\mathbf{9 . 5 6} \pm 0.16$ & $854.06 \pm 1.84$ & $1.574 \pm 0.064$ & $272.47 \pm 2.54$ & $45.41 \pm 0.42$
\end{tabular}

Table 6.9: Results for 15 seconds of cellular, scenario 3, in laboratory conditions.

In the Table 6.7 for scenario 1 with the Cell Solution, in the Table 6.8 for scenario 2 and in the Table 6.9 for scenario 3 are presented values for the average delay (in milliseconds), the average jitter (in milliseconds), the average bitrate (in Kilobit per second), and the average packet rate (in packets per second). For the Base Solution the average delay and the average jitter were not presented due to the nature of the tool D-ITG that would not provide correct and useful values for these metrics due to the loss of connection incorporated in this scenario for a considerable time window. Analysing the values in these tables, it is possible to conclude that scenario 2 presents the overall best results as it would be expected based on the characteristics of the WAVE technology compared to the cellular technology in the evaluated conditions. Comparing these values for the WAVE case, scenario 2, and for the 15 seconds of cellular, scenario 3, to the Cell Solution it is possible to conclude that, as expected, the overall values obtained in the Cell Solution are kept in a range where the average is influenced by the less optimal values that the use of cellular implies, as seen in the results of Table 6.9. This is noticeable mainly in the delay and jitter for all test cases, showing the difference of a direct WAVE connection to the VANET or the need to use a commercial network to go through the internet in order to reach the LMA, and in the bitrate and packet rate for the test $\mathrm{C}$ and test D cases showing the impact of the cellular network for cases where the packet rate is high, as previously mentioned. 
Based on the results for a constant WAVE connection for each test case we can see that this technology, under the evaluation conditions, introduces few to non packet loss in the connection, for all test cases. Analysing the results for the cellular connection via a commercial network we can see a considerable packet loss that was introduced by the use of the cellular technology, only for a time window of 15 seconds. Based on these results, we can conclude that the cellular network does not perform as desired when subjected to tests with a high packet rate (such as tests $\mathrm{C}$ and $\mathrm{D}$ ), presenting packet losses of $7.74 \%$ in test $\mathrm{C}$ and of $9.56 \%$ in test D. These losses do not come directly from the solution implemented in the VANET and so, to better interpret the real losses caused by the proposed mobility solution, we can subtract the packet losses observed in this scenario to the one presented in scenario 1 for the Cell Solution. As such, the real values for the percentage of packet loss originated from the solution implemented in the mobility protocol correspond to the ones presented in Figure 6.4 ("Adjusted Values") along with the ones presented before. The packet loss "Adjusted Values" correspond to: $1.39 \%$ for test A; $1.81 \%$ for test $\mathrm{B} ; 12.76 \%$ for test C; and, $11.97 \%$ for test D.

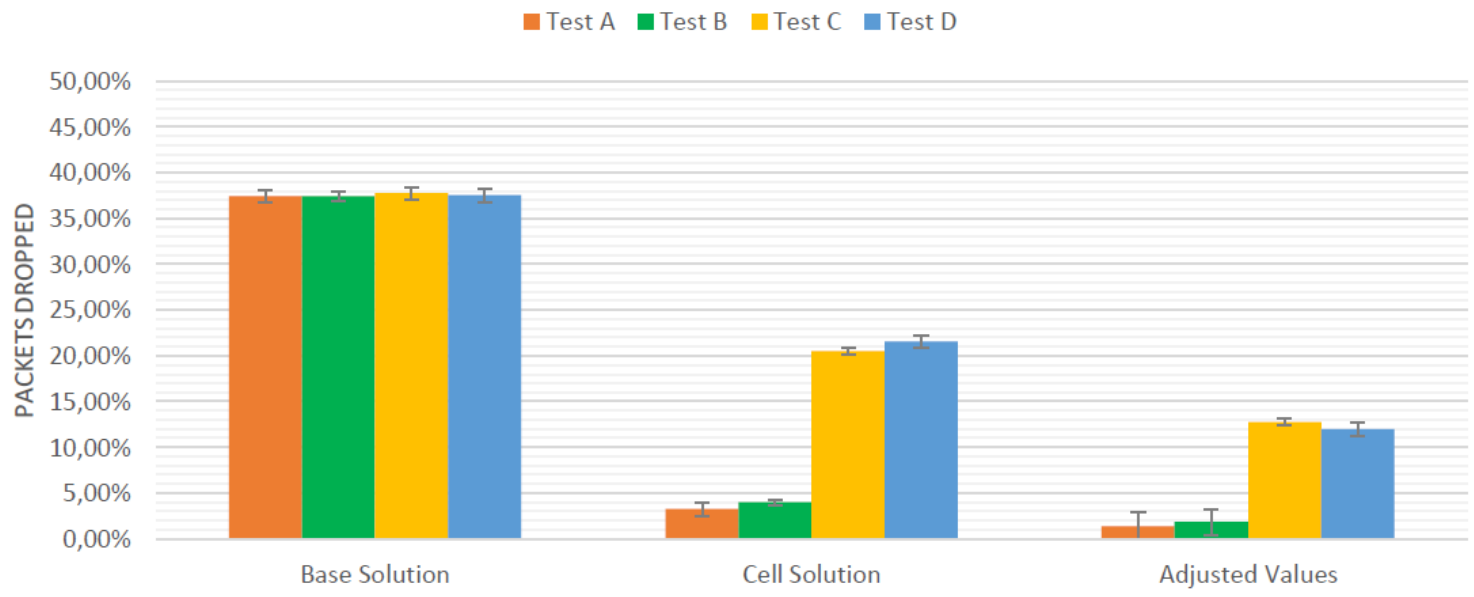

Figure 6.4: Average packet loss in laboratory conditions for both the Base Solution and the Cell Solution in scenario 1 and the "Adjusted Values".

As mentioned before the commercial cellular network does not perform well with high packet rate traffic. Nevertheless, with the "Adjusted Values", the difference between the Cell Solution and scenario 4, it is still noticeable a dissimilarity in the percentage of packets dropped for tests A and B to the tests C and D. Further in Section 6.2.3 will be provided a deeper analysis for the cause of such difference. Finally, comparing the " Adjusted Values", which provide the percentage of packets dropped for the performance of the VANET with the cellular solution not considering the commercial cellular network inherent losses, to the results from the Base Solution we can calculate the gain of the solution for each test, and they are: for test A 96.30\%; for test B 95.16\%; for test C 66.20\%; and, for test D $68.08 \%$. 


\subsubsection{Real-World Tests}

Tests in real-world conditions introduce non-controlling factors like additional vehicular traffic, pedestrians, vehicle velocity, and weather conditions that may affect the performance of the VANET. Also, with these factors it is not possible to guarantee a synchronized timeline for the tests, although the evaluation scenario will be the same as before, presented in Figure 6.2, allowing a somewhat simpler comparison between the real-world and laboratory results. The time windows in which the mobile node is able to be connected to a WAVE RSU, and is without connection for the Base Solution or using cellular for the Cell Solution cannot be precisely maintained equal between runs, therefore the time windows for the use of cellular will correspond to time windows of $[15 ; 30]$ seconds. As intended, the tests represent a real life scenario were the VANET platform is running and reacting automatically, based on the movement of the vehicle equipped with the OBU. Taking into account the evaluation scenario, a vehicle stopped in a semaphore where it has a connection to a WAVE RSU will start to move along the road and consequently loses the WAVE connection for a certain time window until it finally reacquires a WAVE connection and eventually stops again in its range.

For the Base Solution in scenario 1, like in the laboratory tests, are presented in Table 6.10 the results for the cases of use of the VANET without the cellular solution, meaning that when the WAVE connection is lost there is no alternative connection (therefore, corresponding to a downtime). The results on packet loss, bitrate and packet rate are presented in Table 6.10.

\begin{tabular}{c|ccc} 
Test & Packet loss $(\%)$ & Bitrate $(\mathrm{kbit} / \mathrm{s})$ & Packet rate $(\mathrm{pkt} / \mathrm{s})$ \\
\hline \hline $\mathrm{A}$ & $\mathbf{4 3 . 1 6} \pm 4.74$ & $56.72 \pm 0.07$ & $4.73 \pm 1.18$ \\
$\mathrm{~B}$ & $\mathbf{4 6 . 1 3} \pm 5.48$ & $80.63 \pm 8.21$ & $13.44 \pm 1.37$ \\
\hline $\mathrm{C}$ & $\mathbf{4 4 . 4 5} \pm 6.14$ & $219.85 \pm 24.35$ & $54.96 \pm 6.09$ \\
$\mathrm{D}$ & $\mathbf{4 6 . 8 2} \pm 3.93$ & $315.56 \pm 23.38$ & $52.59 \pm 3.88$
\end{tabular}

Table 6.10: Results for the Base Solution in real-world conditions.

Taking into consideration that these values refer to a time window of 50 seconds, where we have a time frame for the loss of connectivity greater than the one we had for the laboratory tests, corresponding to 15 seconds up to 30 seconds, and also having real-world factors, such as pedestrians walking along the road and/or the sidewalk passing between the line-of-sight of the RSU and the vehicle, it can be concluded that the increased values for the packet loss, noticeable in Table 6.10, are justifiable. These values, in laboratory conditions were around $[37 \% ; 38 \%]$ and, for the tests with real-world conditions go from $43.16 \%$ to $46.82 \%$.

The Cell Solution in scenario 1, under real-world conditions, is being evaluated by the example scenario provided above. The results are presented in Table 6.11.

The results obtained for the tests in a real-world scenario with the cellular solution were in average as low as $1.68 \%$ for test $\mathrm{A}$ and $3.47 \%$ for test $\mathrm{B}$ for the percentage in packets dropped. With these values it is possible to conclude that the performance is better than the one for the tests conducted in laboratory conditions (noticing a decrease of $1.57 \%$ for test $\mathrm{A}$ and $0.52 \%$ for test $\mathrm{B}$ ), but for tests $\mathrm{C}$ and $\mathrm{D}$ the average of percentage of packet loss were worse but still close to the ones obtained in laboratory conditions being $22.20 \%$ and $24.16 \%$, 


\begin{tabular}{c|ccccc} 
Test & Packet loss $(\%)$ & Delay $(\mathrm{ms})$ & Jitter $(\mathrm{ms})$ & Bitrate $(\mathrm{kbit} / \mathrm{s})$ & Packet rate $(\mathrm{pkt} / \mathrm{s})$ \\
\hline \hline A & $\mathbf{1 . 6 8} \pm 0.12$ & $10.71 \pm 3.02$ & $0.721 \pm 0.067$ & $98.11 \pm 0.12$ & $24.53 \pm 0.03$ \\
B & $\mathbf{3 . 4 7} \pm 0.49$ & $14.89 \pm 1.48$ & $0.925 \pm 0.240$ & $144.46 \pm 0.81$ & $24.08 \pm 0.14$ \\
\hline C & $\mathbf{2 2 . 2 0} \pm 3.19$ & $94.44 \pm 54.20$ & $0.620 \pm 0.121$ & $307.77 \pm 12.61$ & $76.94 \pm 3.15$ \\
D & $\mathbf{2 4 . 1 6} \pm 4.31$ & $79.24 \pm 34.99$ & $0.640 \pm 0.117$ & $449.99 \pm 25.59$ & $75.00 \pm 4.27$
\end{tabular}

Table 6.11: Results for the Cell Solution in real-world conditions.

respectively, noticing a slight increase of $1.7 \%$ for test $\mathrm{C}$ and $2.63 \%$ for test D. Considering the conditions of the tests and the performance of the cellular network for high packet rates it can be concluded that the results obtained in real-world conditions were more than expected.

It is also provided results for scenario 2 and scenario 3 in order to allow a better understanding of the impact of such real-world conditions in these scenarios, also comparing to the laboratory conditions. The results for scenario 2 can be observed in Table 6.12 and for scenario 3 in Table 6.13 .

\begin{tabular}{c|ccccc} 
Test & Packet loss $(\%)$ & Delay $(\mathrm{ms})$ & Jitter $(\mathrm{ms})$ & Bitrate $(\mathrm{kbit} / \mathrm{s})$ & Packet rate $(\mathrm{pkt} / \mathrm{s})$ \\
\hline \hline A & $\mathbf{1 . 3 6} \pm 2.67$ & $39.10 \pm 2.10$ & $0.096 \pm 0.177$ & $98.43 \pm 2.67$ & $24.61 \pm 0.67$ \\
B & $\mathbf{3 . 2 4} \pm 6.35$ & $38.23 \pm 0.06$ & $0.010 \pm 0.003$ & $144.83 \pm 9.50$ & $24.14 \pm 1.58$ \\
\hline C & $\mathbf{3 . 4 0} \pm 6.67$ & $38.43 \pm 0.04$ & $0.007 \pm 0.001$ & $382.01 \pm 26.37$ & $95.50 \pm 6.59$ \\
D & $\mathbf{4 . 3 4} \pm 5.47$ & $32.31 \pm 0.36$ & $0.008 \pm 0.001$ & $567.66 \pm 32.55$ & $94.61 \pm 5.42$
\end{tabular}

Table 6.12: Results for 50 seconds of WAVE connection, scenario 2, in real-world conditions.

\begin{tabular}{c|ccccc} 
Test & Packet loss $(\%)$ & Delay $(\mathrm{ms})$ & Jitter $(\mathrm{ms})$ & Bitrate $(\mathrm{kbit} / \mathrm{s})$ & Packet rate $(\mathrm{pkt} / \mathrm{s})$ \\
\hline \hline A & $\mathbf{1 . 5 5} \pm 1.86$ & $23.38 \pm 6.29$ & $1.200 \pm 0.213$ & $100.42 \pm 0.10$ & $25.11 \pm 0.03$ \\
B & $\mathbf{2 . 4 1} \pm 1.93$ & $17.77 \pm 6.38$ & $1.068 \pm 0.353$ & $147.09 \pm 2.79$ & $24.52 \pm 0.46$ \\
\hline C & $\mathbf{5 . 6 5} \pm 1.52$ & $815.42 \pm 10.76$ & $1.360 \pm 0.018$ & $187.19 \pm 1.07$ & $46.80 \pm 0.27$ \\
D & $\mathbf{8 . 9 2} \pm 1.24$ & $842.35 \pm 13.02$ & $1.471 \pm 0.017$ & $273.48 \pm 2.07$ & $45.58 \pm 0.34$
\end{tabular}

Table 6.13: Results for 15 seconds of cellular, scenario 3, in real-world conditions.

Contrary to what was the case in laboratory conditions, where the impact in the percentage of packets dropped for the WAVE connection was null, in this case the WAVE connection will have impact in the overall performance of the VANET (as shown by the results obtained in Table 6.12). This deterioration of the performance of WAVE is due to real-world environment factors now present in the experiments, having results for the average of the percentage of packets dropped of $1.36 \%$ (for test A), being the lowest, to $4.34 \%$ for test $\mathrm{D}$, being the highest.

For the cellular connection the values presented are a slightly better, compared to the ones obtained in laboratory conditions (presented in Table 6.9). This may be due to a better signal strength verified outside the laboratory. Nevertheless, it also presents some impact in the percentage of packets dropped with results of $1.55 \%$ for test A, being the lowest, to $8.92 \%$ for test $\mathrm{D}$, being the highest. 
Since the time frame for the use of cellular varies from run to run, and all the other conditions also contribute to the entropy in these scenarios, it will not be subtracted the direct impact of scenario 2 and scenario 3 to the Cell Solution. Therefore the final results of the performance of the cellular solution developed and implemented in this VANET are the ones presented for the Cell Solution, in Table 6.11. It is provided in Figure 6.5 a visual illustration of the results obtained for the packet loss in the Base Solution (scenario 1) for the performance of the VANET without using cellular, and in the Cell Solution (scenario 1) the main scenario that evaluates the performance of the VANET with cellular (representing the solution implemented in this dissertation) under real-world conditions.

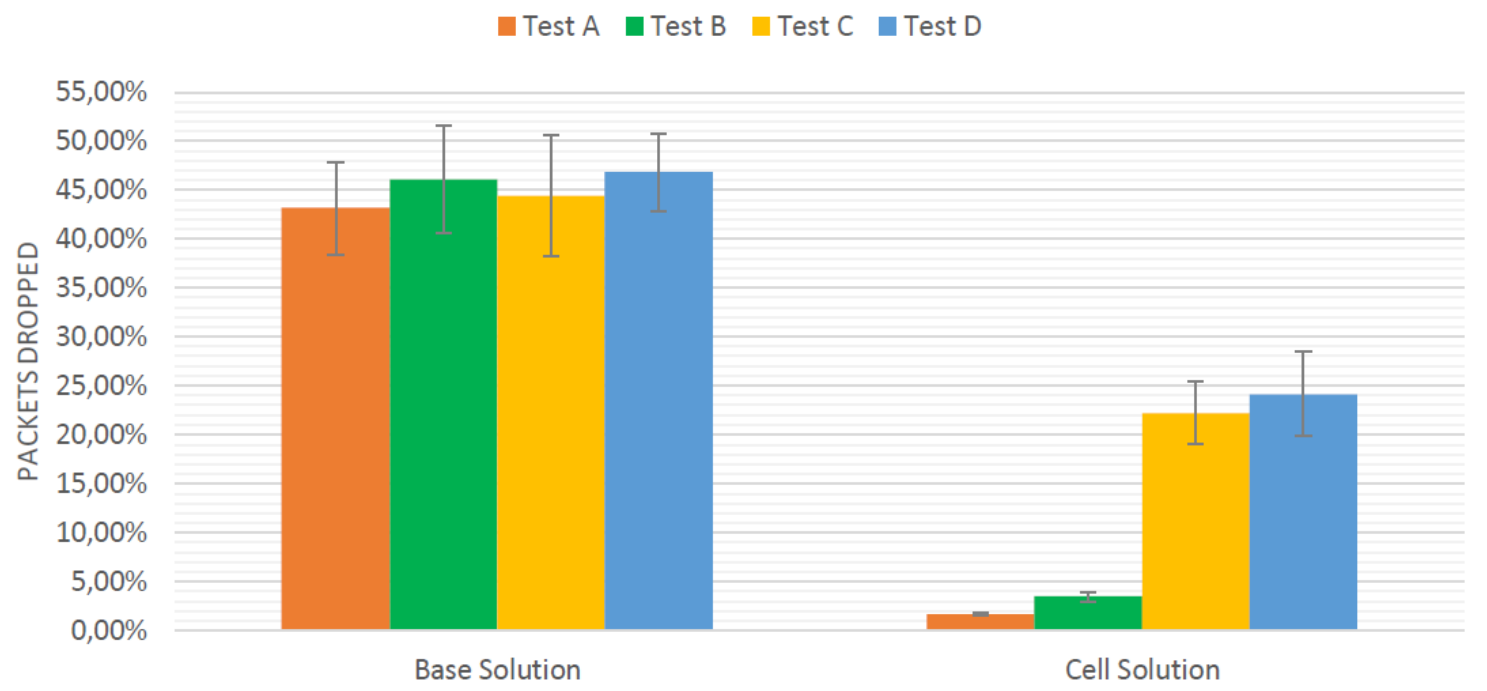

Figure 6.5: Average packet loss in real-world conditions for the Base Solution and the Cell Solution.

\subsubsection{Analysing the performance over time}

This section presents an analysis of the number of packets dropped overtime for the Cell Solution in scenario 1, representing the performance of the VANET with the cellular solution, with the parameters 25 packets per second with 500 bytes per packet (test A), and 25 packets per seconds with 750 bytes per packet (test B). It aims to provide a better picture of the performance over the time window of the experiment execution and, at the same time, to provide a justification for the cause of the losses that are still noticeable in the results.

The 100 packets per second cases (test $\mathrm{C}$ and test $\mathrm{D}$ ) will not be analyzed because, as already concluded in subsection 6.2.1, the cellular network does not perform well with high rates of packets, and therefore, their graphical representation, would not contribute in any meaningful way to the conclusions of the topic discussed in this section.

Next, are provided illustrations related to the number of packets dropped for the best case and also the worst case obtained in the runs for test A with the Cell Solution in Figure 6.6, and in the runs for test B with the Cell Solution in Figure 6.7. 


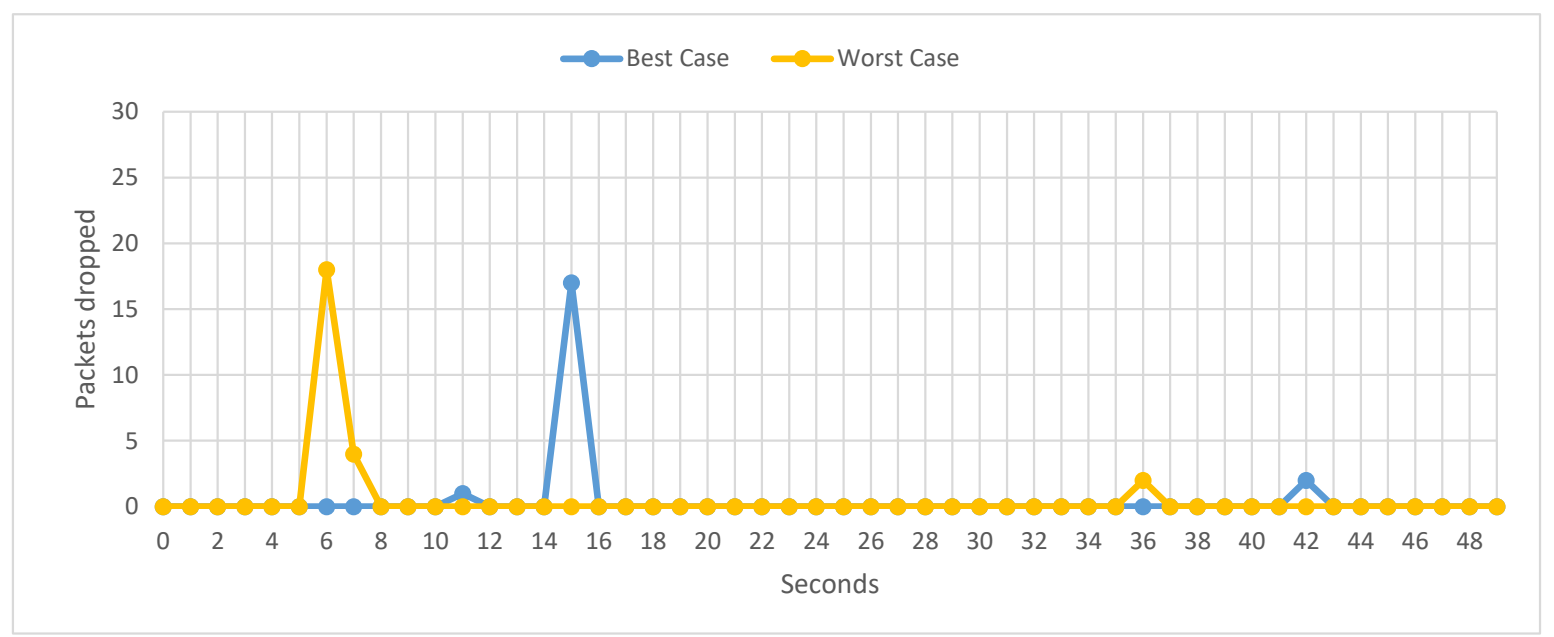

Figure 6.6: Best and Worst case for test A, in scenario 1 with the Cell Solution.

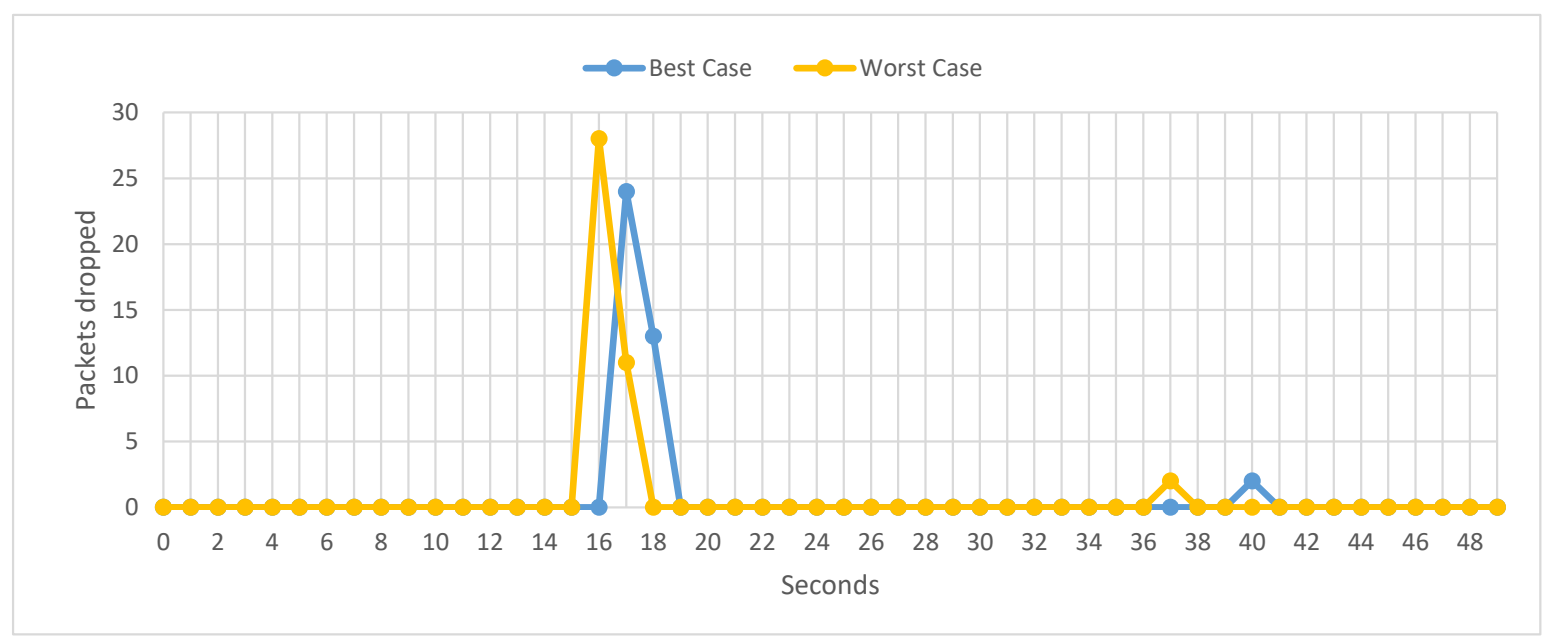

Figure 6.7: Best and Worst case for test B, in scenario 1 with the Cell Solution.

Analysing both Figure 6.6 and Figure 6.7 we can see that, as mentioned before, the time window synchronization for the use of cellular in each individual run cannot be guaranteed. Nevertheless, these different time windows do not affect the conclusions that will be obtained by the analysis of the figures, as it can be deduced from the figures showing that the number of packets dropped during the time the VANET makes use of the cellular connection is equal to zero.

Analysing more in detail the Figure 6.6, illustrating the results for a packet rate of 25 and packet size of 500 bytes (test A), it is possible to see that when a WAVE connection is lost it occurs an handover to cellular and the number of packets dropped during this process is substantial, especially when compared to the number of packets dropped in the handover between the cellular and WAVE (when a WAVE connection is again established). In the first handover, WAVE to cellular, that occurs at approximately instant $\mathrm{t}=5$ seconds for the worst case and $t=14$ seconds for the best case, and the number of packets dropped corresponds to $22(18+4)$ in a total period of 2 seconds for the worst case, and corresponds to 17 for a total 
period of 1 second for the best case. In the second handover process, from cellular to WAVE, that occurs at approximately instant $\mathrm{t}=35$ seconds for the worst case and $\mathrm{t}=41$ seconds for the best case, the number of packets dropped corresponds to 2 for a total period of 1 second for both cases. During the other periods of time, where the connections being used correspond to cellular or WAVE, there are no losses (ignoring a specific case where 1 packet was lost during the first period of WAVE connection in the best case for test A).

Analysing more in detail the Figure 6.7, illustrating the results for a packet rate of 25 and packet size of 750 bytes (test B), we can observe similar performances as the ones obtained for test A. However, in these runs, the number of packets dropped was higher in the handover from WAVE to cellular, that occurs at approximately instant $t=15$ seconds for the worst case and $t=16$ seconds for the best case, and corresponds to 39 packets dropped for the worst case and to 37 packets dropped for the best case, for a total period of 2 seconds in both cases. This increase of packets dropped is associated to the increase in the packet size. For the second handover process, from cellular to WAVE, that occurs at approximately instant $\mathrm{t}=36$ seconds for the worst case and $t=39$ seconds for the best case, the values were equal to the ones observed in the previous test A experiments, being of 2 packets dropped for a total period of 1 second.

We can, therefore, conclude that the main reason for the losses, observed in the experiments, happens in the WAVE to cellular handover process. This fact may be due to the tx_ring size, of the WAVE driver, as when the route is changed to cellular the packets that are already in the ring buffer of the WAVE interface, waiting to be sent, are lost. The cellular driver has a small buffer size and, consequently, presents smaller packet dropped values. As expected, with the increase in the size of the packets the losses become more significant. The ring buffer size was not changed, due to characteristics of the equipment available, and so that this solution presents itself as not requiring additional changes, in the buffers, that are defined by the drivers of the respective interface.

After an exhaustive evaluation process, tackling different network parameters and handover situations, it is concluded that with the new cellular solution the VANET is indeed capable of maintaining a connection between the nodes (OBU and LMA) via a commercial cellular network, and without losing packets during the cellular time window under ideal conditions (taking into account the limitations of the cellular networks). Before these improvements verified in the Cell Solution, under scenarios like the one studied in scenario 1, with the Base Solution the system would be subject to a downtime (no connection was possible).

\subsubsection{Disconnect Mechanism Tests}

Let us now analyze and discuss results for different values of disconnect threshold in the implemented disconnect mechanism, so that conclusions on a pro-active reaction of the system may be possible. This aims to allow the avoidance of significant packet losses due a WAVE connection with weak signal, as mentioned in subsection 5.2.1.

Due to the conclusions presented in subsection 6.2.1 and subsection 6.2.2, we will only consider the network parameters of tests $\mathrm{A}$ and B, i.e., packet rate of 25 and packet size of 500 bytes and 750 bytes, respectively. These new tests will be performed following scenario 1 with the Cell Solution in real-world conditions, corresponding to real-world tests of the VANET using cellular capabilities. As a reminder, the scenario consists in a stable connection to a 
WAVE RSU as the test begins followed by the loss of such connection and the consequent use of cellular during an interval with duration of [15;30] seconds and, after that, a connection to a WAVE RSU is again established, resulting in a total duration time of 50 seconds for the test run. Contrary to the previous experiments, these tests were executed using a different commercial cellular provider, that being $M E O$ (previously know in Portugal as TMN). Three different sets of threshold were evaluated:

- For the first set, the WAVE disconnect threshold is defined to RSSI $=0$, therefore, the new disconnect mechanism will force a disconnect at values of RSSI $<10$. The connect is defined to RSSI $\geq 30$;

- For the second set, the WAVE disconnect threshold is defined to RSSI $=10$, therefore, the new disconnect mechanism will force a disconnect at values of RSSI $<20$. The connect is defined to RSSI $\geq 30$. This set corresponds to the default values implemented and therefore used in all the tests conducted in the previous subsections (either in laboratory or real-world);

- For the third set, the WAVE disconnect threshold is defined to RSSI $=20$, therefore, the new disconnect mechanism will force a disconnect at values of $\mathrm{RSSI}<30$. The connect is defined to RSSI $\geq 40$;

Table 6.14, Table 6.15 and Table 6.16 present the results for each respective set presented above, as follows:

\begin{tabular}{c|ccccc} 
Test & Packet loss (\%) & Delay $(\mathrm{ms})$ & Jitter $(\mathrm{ms})$ & Bitrate $(\mathrm{kbit} / \mathrm{s})$ & Packet rate $(\mathrm{pkt} / \mathrm{s})$ \\
\hline A & $\mathbf{1 1 . 0 2} \pm 2.07$ & $17.34 \pm 5.99$ & $1.056 \pm 0.139$ & $88.64 \pm 2.21$ & $22.16 \pm 0.55$ \\
B & $\mathbf{1 3 . 2 1} \pm 1.39$ & $9.66 \pm 14.38$ & $1.310 \pm 0.072$ & $129.77 \pm 1.93$ & $21.63 \pm 0.32$
\end{tabular}

Table 6.14: Results for tests with a disconnect for RSSI $<10$.

\begin{tabular}{c|ccccc} 
Test & Packet loss (\%) & Delay $(\mathrm{ms})$ & Jitter $(\mathrm{ms})$ & Bitrate $(\mathrm{kbit} / \mathrm{s})$ & Packet rate $(\mathrm{pkt} / \mathrm{s})$ \\
\hline A & $\mathbf{7 . 9 6} \pm 0.92$ & $17.64 \pm 3.26$ & $1.123 \pm 0.155$ & $91.83 \pm 0.91$ & $22.96 \pm 0.23$ \\
B & $\mathbf{9 . 3 5} \pm 0.05$ & $17.93 \pm 1.10$ & $1.265 \pm 0.122$ & $135.68 \pm 0.08$ & $22.61 \pm 0.01$
\end{tabular}

Table 6.15: Results for tests with a disconnect for RSSI $<20$.

\begin{tabular}{c|ccccc} 
Test & Packet loss $(\%)$ & Delay $(\mathrm{ms})$ & Jitter $(\mathrm{ms})$ & Bitrate $(\mathrm{kbit} / \mathrm{s})$ & Packet rate $(\mathrm{pkt} / \mathrm{s})$ \\
\hline A & $\mathbf{7 . 5 1} \pm 0.43$ & $13.21 \pm 2.36$ & $1.182 \pm 0.069$ & $92.20 \pm 0.61$ & $23.05 \pm 0.15$ \\
B & $\mathbf{9 . 3 2} \pm 0.20$ & $16.16 \pm 6.54$ & $1.434 \pm 0.208$ & $135.72 \pm 0.29$ & $22.62 \pm 0.05$
\end{tabular}

Table 6.16: Results for tests with a disconnect for RSSI $<30$.

The results show that the performance of the VANET when the disconnect mechanism is defined to trigger the handover from a WAVE connection to the cellular connection at a mean RSSI inferior to 10 present more packet loss compared to the other disconnect cases. 
Therefore, the sooner the disconnect mechanism triggers the handover process the better the performance of the VANET in terms of packet loss, that is justified by the quality signal of the WAVE connection before the handover process. Moreover, the difference of performance between the tests with a disconnect at RSSI $<20$ and the tests with a disconnect at RSSI $<30$ can be considered similar, taking into account the conditions for these test runs, except for a visible slightly better performance related to the delay verified in the case where the disconnect occurs at RSSI value inferior to 30 instead of occurring at RSSI value inferior to 20 , and that could be explained by the evaluation conditions, more specifically interference's in the environment at the moment of the tests for the disconnect at RSSI value inferior to 20.

An interesting fact that can also be retained from these experiments is, for example, the significant increase in the percentage of packet loss when comparing the results of the tests A and B of Table 6.15 (7.96\% for test A, and $9.35 \%$ for test B) to the ones obtained in the previous section, with a different network service provider, presented in Table 6.11 (1.68\% for test $\mathrm{A}$, and $3.47 \%$ for test B). Both experiments were conducted under real-world conditions but, it should be noted that, for the ones presented in this subsection the cellular provider used was different, as mentioned, and the conditions had an increase in traffic and pedestrian activity during the realization of these tests compared to the tests of the previous section (tests conducted at different days, maintaining the setup and location), factors that may provide a justification to the difference in performance values obtained. Moreover, before taking final conclusions further insights for the analysis of the performance of these tests will be provided next.

Illustrated in Figure 6.8 is the graphical representation of the results for a run of each of the three sets with the conditions of test A, 25 packets per second with 500 bytes per packet (the other runs provided similar results). And illustrated in Figure 6.9 is the graphical representation of the results for a run of each of the three sets with the conditions of test $\mathrm{B}$, 25 packets per second with 750 bytes per packet.

Analysing the results presented in Figure 6.8 and Figure 6.9, in either figures a pattern can be observed: in both tests (A and B), the higher the disconnect threshold the sooner the handover is triggered in the VANET. Also for the set where the disconnect only happens when the RSSI becomes lower than 10 (the lowest tested disconnect threshold value) it can be noted that packet loss starts to occur before the actual handover (packet loss is noticeable starting at instant $\mathrm{t}=22$ for test $\mathrm{A}$, the disconnect only occurs at approximately instant $\mathrm{t}=27$; packet loss is noticeable starting at instant $\mathrm{t}=19$ for test $\mathrm{B}$, the disconnect only occurs at approximately instant $\mathrm{t}=28$;), meaning that with low WAVE quality signal the connection starts to lose packets only after the handover is triggered and we see the expected tendency of the packet drop due to the respective handover from WAVE to cellular. These conclusions come as expected, and confirm the choice of triggering the disconnect from WAVE to cellular when the RSSI is lower than 20. With this methodology we can take advantage of the WAVE connection until before the connection starts to underperform severely at which moment we change to the cellular connection. This (second set) was the methodology used in all the tests performed in the previous sections. 


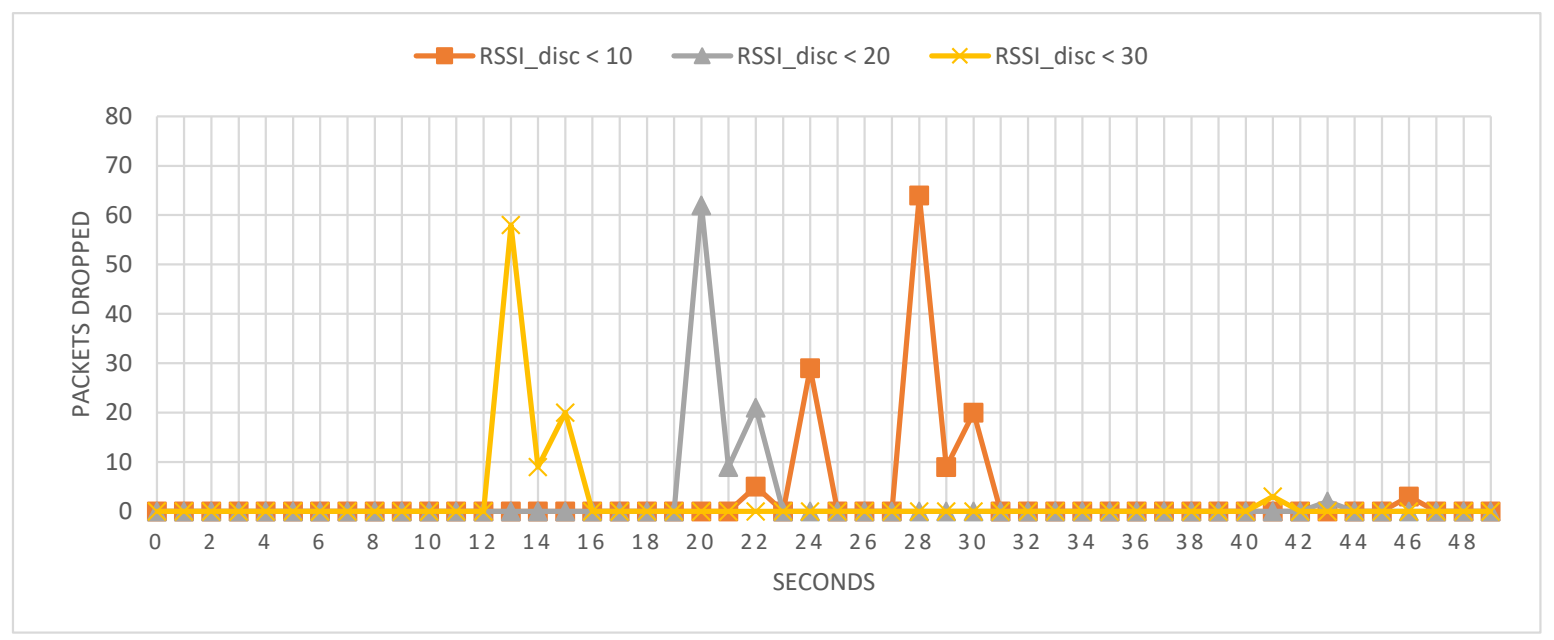

Figure 6.8: Packets dropped for tests A.

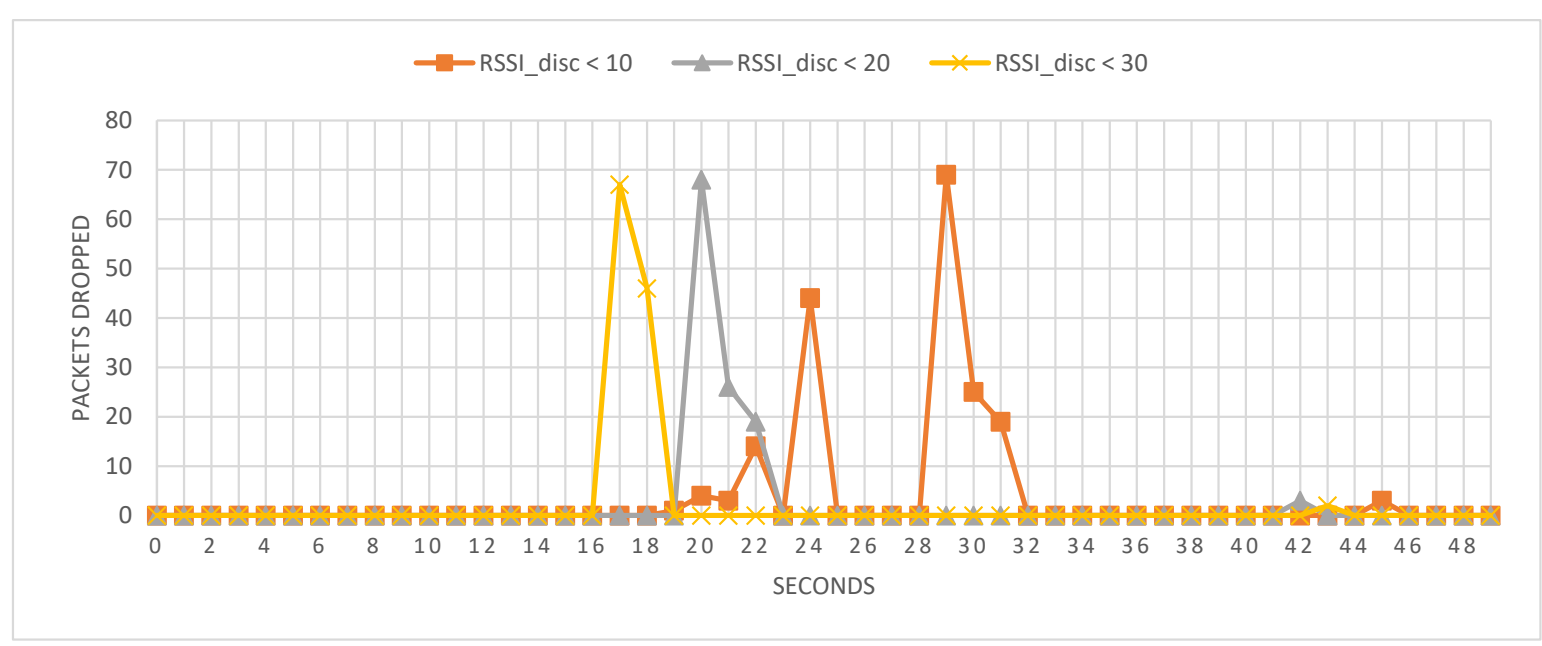

Figure 6.9: Packets dropped for tests B.

\subsection{Final Considerations}

This chapter presented the equipment used in the experiments, the scenarios for the tests in laboratory and real-world conditions, and its results. The tests presented in this chapter were conducted with the main goal of evaluating the work developed in this dissertation and providing insights for improvements.

From the laboratory tests it was demonstrated that the cellular solution performed successfully, therefore, providing a connection via the commercial cellular network when no other connection was available, as intended. Moreover, it was demonstrated that the use of a cellular connection in low throughput scenarios usually presents no loss of packets, but for high throughput scenarios the cellular network may not provide the best performance, nevertheless still presenting itself as a well justifiable option.

It were also conducted tests in a real-world scenario, in order to validate the correct functioning of the system in such conditions. It was also provided an analysis of the performance 
of the system during the time window of the tests where, when excluding the cellular network inherent losses for high throughput scenarios, it was demonstrated that the loss of packets that occurs happens during the handover processes, specifically during the handover process from WAVE to cellular, and it were not observed packet losses during the use of cellular. It was, therefore, validated the performance of the VANET with the cellular solution in such real-world conditions.

Finally, in order to compare and justify the choice of values for the implemented disconnect mechanism, deciding at what disconnect threshold RSSI value to trigger the handover process from the WAVE connection to the cellular connection, tests were executed for three different sets of values for the disconnect mechanism. They provided conclusions on the set of values to use, allowing the disconnect mechanism to trigger the handover process before the WAVE connection starts to show packet losses due to its quality of signal.

The next chapter provides final conclusions and some guidelines for possible future work. 


\section{Chapter 7}

\section{Conclusions and Future Work}

This dissertation developed a solution to provide a VANET with cellular capabilities, to be used when no other access technology (WAVE or Wi-Fi) connection was possible. For that, the idea was to explore the high availability of cellular networks without requiring any specific contribution by the cellular network providers or the end-users, therefore resulting in higher network connectivity for the VANET.

In order to fulfil this goal, it was proposed an architecture that would make use of a direct communication between the OBUs and the LMA via the cellular network. For that, several modifications were required in order to overcome the challenges of such network architecture, such as the NAT mechanisms used by commercial cellular networks or the adaptation of the mobility platform for the handover between the existing technologies and the new cellular technology approach, among others. The list of modifications include: (1) the modification of the base vehicular mobility platform with the implementation of improvements related to the dynamic configuration of end-to-end routes for the communication between the end-user and the Internet; (2) the implementation of new mechanisms allowing the UDP encapsulation and routing of packets that are to be sent via a commercial cellular network from the OBU to the LMA or vice versa; (3) or the management process to keep the cellular connections updated with respect to the vehicular network.

The validation of the proposed architecture was conducted based on the performance of the VANET under different conditions, both in laboratory and real-world. These tests with different parameters and also different scenarios, such as different traffic loads, allowed for a better analysis of the performance of the VANET, with and without the integration of the cellular technology. Additionally, and because a new wireless interface was added in the OBU, the new disconnect mechanism with respect to the cellular interface was also evaluated. The results showed that significant packet losses occur during periods of weak signal in the WAVE connection, and therefore, the handover process from WAVE to cellular is triggered by the disconnect mechanism before the WAVE connection starts to present low performance.

The integration of cellular capabilities was accomplished, providing a new access network option, allowing the users connected to the OBUs to communicate to the Internet via the VANET network when no connection to a RSU is possible, increasing the coverage and, therefore, the availability of the system and overall Quality of Experience (QoE) to the enduser. 


\subsection{Future Work}

Although the objective of this dissertation was accomplished with several improvements implemented along the way, there is still space for improvement. Moreover, this work paves the way for the study and development of new solutions in the scope of vehicular networks, which can be explored in new coming cellular architectures. Therefore, a list of topics for future work is enumerated as follows:

- Development of a "packet drop awareness mechanism" that triggers the handover between access technologies: as seen in the evaluation tests of the disconnect mechanism, the WAVE connection starts to underperform below a given signal quality resulting in the loss of packets. This situation could be explored to trigger the handover process;

- Further optimization of the handover process between WAVE and the cellular solution, possibly via pro-active network stack buffering: as seen in the evaluation process, the main reason for packet loss lies in the handover process between WAVE and cellular technologies, therefore further optimization, possibly focused on the ring buffers of the network adapter, can be studied and applied to improve the performance of the VANET;

- Use of cellular solution in parallel with WAVE and/or Wi-Fi to send specific critical traffic, aiming to improve the QoS: the solution implemented in this dissertation took an approach to be used as a last resort, when no WAVE or Wi-Fi connections are available, but it can also be studied possible improvements to this solution for traffic division, according to priorities, to be sent via the cellular network due to its availability, even when other connections are available;

- Integration of $5 \mathrm{G}$ technologies to provide new features to the VANET: the $5 \mathrm{G}$ is the next step for cellular technologies, not focusing only in the cellular aspect but also in the different types of applications as proved by the current push by academic and industry entities for a standardization. Technological integration's facilitated by these 5G technologies may, for example, be edge-computing capabilities that should allow an exponential increase in functionalities and performance of mobile nodes. 


\section{Bibliography}

[1] B. Toghi, M. Saifuddin, H. N. Mahjoub, M. O. Mughal, Y. P. Fallah, J. Rao, and S. Das. Multiple Access in Cellular V2X: Performance Analysis in Highly Congested Vehicular Networks. In 2018 IEEE Vehicular Networking Conference (VNC), pages 1-8, Dec 2018.

[2] H. Zhou, H. Wang, X. Chen, X. Li, and S. Xu. Data Offloading Techniques Through Vehicular Ad Hoc Networks: A Survey. IEEE Access, 6:65250-65259, 2018.

[3] Sherali Zeadally, Ray Hunt, Yuh-Shyan Chen, Angela Irwin, and Aamir Hassan. Vehicular ad hoc networks (VANETS): status, results, and challenges. Telecommunication Systems, 50(4):217-241, Aug 2012.

[4] TransportXtra. VANET Example, 2017. [Online; accessed October 23, 2019].

[5] P. HemaLatha M. Newlin Rajkumar, M. Nithya. Overview of VANET with its features and security attacks. IRJET, 03:137-142, January 2016.

[6] Gurpreet Singh Manjot Kaur, Sukhman Kaur. Vehicular Ad Hoc Networks. JGRCS, 3:61-64, March 2012.

[7] Felipe Domingos Da Cunha, Azzedine Boukerche, Leandro Villas, Aline Carneiro Viana, and Antonio A. F. Loureiro. "Data Communication in VANETs: A Survey, Challenges and Applications". Research Report RR-8498, INRIA Saclay ; INRIA, March 2014.

[8] Subhas Misra, Isaac Woungang, and Sudip Misra. Guide to Wireless Ad Hoc Networks. Springer, 012009.

[9] Saif Al-Sultan, Moath M. Al-Doori, Ali H. Al-Bayatti, and Hussien Zedan. A comprehensive survey on vehicular Ad Hoc network. Journal of Network and Computer Applications, $37: 380-392,2014$.

[10] Tanuja K, Sushma M, Bharathi M, and Arun H. A Survey on VANET Technologies. International Journal of Computer Applications, 121:1-9, 072015.

[11] Manjusha Deshmukh and Divya Dinesh. Challenges in Vehicle Ad Hoc Network (VANET). IJETMAS, 2:76-88, 122014.

[12] Ghassan Samara and Yousef Al-Raba'nah. Security Issues in Vehicular Ad Hoc Networks (VANET): a survey. IJSAR, 2:50-55, 122015.

[13] G. E. M. Zhioua, H. Labiod, N. Tabbane, and S. Tabbane. Cellular Content Download through a Vehicular Network: I2V Link Estimation. In 2015 IEEE 81st Vehicular Technology Conference (VTC Spring), pages 1-6, May 2015. 
[14] M.S. Anwer and C.A. Guy. Survey of VANET Technologies. J. Emerg. Trends Comput. Inf. Sci, 5:661-671, 092014.

[15] A. Fitah, A. Badri, M. Moughit, and A. Sahel. Performance of DSRC and WIFI for Intelligent Transport Systems in VANET. Procedia Computer Science, 127:360 - 368, 2018.

[16] Pimmy Gandotra and Rakesh Kumar Jha. Device-to-Device Communication in Cellular Networks: A Survey. Journal of Network and Computer Applications, 71:99 - 117, 2016.

[17] M. Jamil, S. P. Shaikh, M. Shahzad, and Q. Awais. 4G: The future mobile technology. In TENCON 2008 - 2008 IEEE Region 10 Conference, pages 1-6, Nov 2008.

[18] Sumit Kumar Mohammad Meraj ud in Mir. Evolution of Mobile Wireless Technology from 0G to 5G. IJCSIT, 6:2545-2551, 2015.

[19] Zhang SJ. Liu EJ. et al. Eze, E.C. Advances in vehicular ad-hoc networks (VANETs): Challenges and road-map for future development. Int. J. Autom. Comput., 13:1-18, Feb 2016.

[20] Gundavelli, et al. Proxy Mobile IPv6. RFC 5213, RFC Editor, August 2008.

[21] Devarapalli, et al. NEMO Basic Support Protocol. RFC 3963, RFC Editor, January 2005.

[22] I. Soto, C. J. Bernardos, M. Calderon, A. Banchs, and A. Azcorra. Nemo-enabled localized mobility support for internet access in automotive scenarios. IEEE Communications Magazine, 47(5):152-159, May 2009.

[23] F. M. M. Castro. Different technologies in Vehicular Networks: Multihoming and Network Coding. Aveiro University, 2016.

[24] N. F. Capela. Intelligent and Transparent Resource Management. Aveiro University, 2017.

[25] Stewart. Stream Control Transmission Protocol. RFC 4960, RFC Editor, September 2007.

[26] T. D. Wallace and A. Shami. A Review of Multihoming Issues Using the Stream Control Transmission Protocol. IEEE Communications Surveys Tutorials, 14(2):565-578, Second 2012.

[27] A. Garcia-Martinez, M. Bagnulo, and I. V. Beijnum. The Shim6 architecture for IPv6 multihoming. IEEE Communications Magazine, 48(9):152-157, Sep. 2010.

[28] F. Castro, A. Martins, N. Capela, and S. Sargento. Multihoming for uplink communications in vehicular networks. In 2017 Wireless Days, pages 230-237, March 2017.

[29] N. Capela and S. Sargento. Machine Learning for Resources Prediction in Multihoming Scenarios. In 2015 IEEE Globecom Workshops (GC Wkshps), pages 1-7, Dec 2015.

[30] Nelson Capela and Susana Sargento. Multihoming and network coding: A new approach to optimize the network performance. Computer Networks, 75:18 - 36, 2014. 
[31] A. Vinel. 3GPP LTE Versus IEEE 802.11p/WAVE: Which Technology is Able to Support Cooperative Vehicular Safety Applications? IEEE Wireless Communications Letters, 1(2):125-128, April 2012.

[32] S. Chen, J. Hu, Y. Shi, and L. Zhao. LTE-V: A TD-LTE-Based V2X Solution for Future Vehicular Network. IEEE Internet of Things Journal, 3(6):997-1005, Dec 2016.

[33] H. Zhou, H. Wang, X. Chen, X. Li, and S. Xu. Data Offloading Techniques Through Vehicular Ad Hoc Networks: A Survey. IEEE Access, 6:65250-65259, 2018.

[34] F. Lin and Y. Liu. An integration of WAVE and LTE wireless transmission in vehicle networks for safety and non-safety messages dissemination. In 2017 3rd IEEE International Conference on Computer and Communications (ICCC), pages 315-320, Dec 2017.

[35] M. Valta, M. Jutila, and J. Jämsä. IEEE 802.11p and LTE as enablers of cognitive vehicle-to-infrastructure communication. In 2015 6th IEEE International Conference on Cognitive Infocommunications (CogInfoCom), pages 71-76, Oct 2015.

[36] A. Benslimane, T. Taleb, and R. Sivaraj. Dynamic Clustering-Based Adaptive Mobile Gateway Management in Integrated VANET - 3G Heterogeneous Wireless Networks. IEEE Journal on Selected Areas in Communications, 29(3):559-570, March 2011.

[37] M. Syfullah and J. M. Lim. Data broadcasting on Cloud-VANET for IEEE 802.11p and LTE hybrid VANET architectures. In 2017 3rd International Conference on Computational Intelligence Communication Technology (CICT), pages 1-6, Feb 2017.

[38] Ghayet el mouna Zhioua, Houda Labiod, Nabil Tabbane, and Sami Tabbane. An Efficient Qos Based Gateway Selection Algorithm for VANET to LTE Advanced Hybrid Cellular Network. In Proceedings of the 15th ACM International Conference on Modeling, Analysis and Simulation of Wireless and Mobile Systems, MSWiM '12, pages 353-356, New York, NY, USA, 2012. ACM.

[39] S. Ucar, S. C. Ergen, and O. Ozkasap. Multihop-Cluster-Based IEEE 802.11p and LTE Hybrid Architecture for VANET Safety Message Dissemination. IEEE Transactions on Vehicular Technology, 65(4):2621-2636, April 2016.

[40] S. Bi, C. Chen, R. Du, and X. Guan. Proper Handover between VANET and Cellular Network Improves Internet Access. In 2014 IEEE 80th Vehicular Technology Conference (VTC2014-Fall), pages 1-5, Sep. 2014.

[41] Marco Gramaglia, Carlos J. Bernardos, and Maria Calderon. Seamless internet 3G and opportunistic WLAN vehicular connectivity. EURASIP Journal on Wireless Communications and Networking, 2011(1):183, 2011.

[42] J. Dias, A. Cardote, F. Neves, S. Sargento, and A. Oliveira. Seamless horizontal and vertical mobility in VANET. In 2012 IEEE Vehicular Networking Conference (VNC), pages 226-233, Nov 2012.

[43] N. Capela, J. Soares, P. Neves, and S. Sargento. An Architecture for Optimized InterTechnology Handovers: Experimental Study. In 2011 IEEE International Conference on Communications (ICC), pages 1-6, June 2011. 
[44] W. Du, M. Zhengxin, Y. Bai, C. Shen, B. Chen, and Y. Zhou. Integrated Wireless Networking Architecture for Maritime Communications. In 2010 11th ACIS International Conference on Software Engineering, Artificial Intelligence, Networking and Parallel/Distributed Computing, pages 134-138, June 2010.

[45] K. Abboud, H. A. Omar, and W. Zhuang. Interworking of DSRC and Cellular Network Technologies for V2X Communications: A Survey. IEEE Transactions on Vehicular Technology, 65(12):9457-9470, Dec 2016.

[46] Shancang Li, Li Da Xu, and Shanshan Zhao. 5G Internet of Things: A survey. Journal of Industrial Information Integration, 10:1 - 9, 2018.

[47] Syed Shah, Ejaz Ahmed, Muhammad Imran, and Sherali Zeadally. 5G for Vehicular Communications. IEEE Communications Magazine, 56:117, 012018.

[48] A. A. Khan, M. Abolhasan, and W. Ni. 5G next generation VANETs using SDN and fog computing framework. In 2018 15th IEEE Annual Consumer Communications Networking Conference (CCNC), pages 1-6, Jan 2018.

[49] A. Hussein, I. H. Elhajj, A. Chehab, and A. Kayssi. SDN VANETs in 5G: An architecture for resilient security services. In 2017 Fourth International Conference on Software Defined Systems (SDS), pages 67-74, May 2017.

[50] A. M. T. Martins. Mobility in vehicular networks with dynamic connectivity and load balancing. Aveiro University, 2015.

[51] C. Gomes, M. Luís, S. Sargento, A. Zúquete, and R. Lopes. Multi-technology vs Singletechnology Architecture for Network Coding in VANETs. In 2018 IEEE Symposium on Computers and Communications (ISCC), pages 878-883, June 2018.

[52] Alessio Botta, Alberto Dainotti, and Antonio Pescapè. A tool for the generation of realistic network workload for emerging networking scenarios. Computer Networks, 56(15):3531-3547, 2012. 